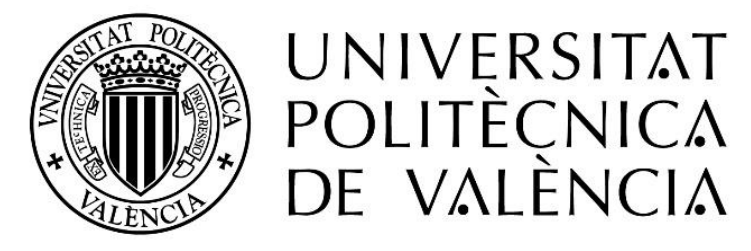

\title{
Dielectric-based Components and Methods for Terahertz Sensing
}

PhD candidate:

Margherita Colleoni

Thesis supervisor:

Borja Vidal Rodríguez

Valencia, Julio 2020 
Alla mia famiglia, fisicamente cosí lontana eppure sempre cosí straordinariamente vicina,

Ai miei amici di sempre, che non hanno ancora capito cosa sto facendo e soprattutto perché

A los mejores amigos que jamás se podrían pedir, que han convertido ir a trabajar en lo mejor del día

A Javi, la mejor persona que ha cruzado mi camino,

Faró del mio meglio per restituirvi tutto quello che mi avete dato e con gli interessi

Grazie, gracias.

"La strada del sucesso é lastricata ... male!"

Margherita Colleoni 
I would like to thank Nanophotonic Technology Centre of Valencia (NTC) and especially my thesis director to give me the opportunity to grow as a professional in the scientific world with the Santiago Grisolia grant.

The journey started there but it was just the beginning. It went around so much until it almost lost its way, but I do not regret any of the steps I have taken. In each one I have discovered new points of view, new people, new areas of knowledge.

Thanks to all those who supported me. You know, this goes for all of you. 
ABSTRACT

In the present work, some aspects of the role of dielectric materials when interacting with $\mathrm{THz}$ waves were investigated. The $\mathrm{THz}$ bandgap, which covers the frequencies from $100 \mathrm{GHz}$ to $30 \mathrm{THz}$, has aroused great interest mainly due to its low energy and its good penetration capacity in some materials of daily use such as fabrics and plastics. The peculiar features of terahertz waves enable their application in various technological fields, especially as scientific tools and for quality control inspection. To advance in the practical application of $\mathrm{THz}$ radiation, the present doctoral thesis researched several paths. Firstly, alternative low-cost methods for manufacturing $\mathrm{THz}$ passive components, in particular, $\mathrm{THz}$ polarizers was treated. The interest in polarization control is derived from the demand for greater control over the physical characteristics of $\mathrm{THz}$ beams. Flexible wire grid polarizers based on the dichroic absorption effect have been investigated. The polarizers have been manufactured using graphite and Galn $_{24.5}$ deployed on ordinary materials used as substrates (paper and polymers such as PVA and PVC). Through a collaboration undertaken with the Chinese research group led by Professor Liu at Tsinghua University, alternative manufacturing processes were researched. All components were simulated through a commercial simulator based on the FIT finite integrals technique (CST Microwave Studio). Secondly, the potential of the fixed delay technique was investigated for rapid sensing of homogeneous and transparent items with possible application to industrial quality inspection. In this scheme, the current variation of the $\mathrm{THz}$ beam at a specific point is related to the variation of its optical parameters, thus it was demonstrated the ability of the method in detecting voids as well as in roughly estimating their volumes under certain conditions. Finally, a different approach for beam profiling based on a dielectric slit aperture was evaluated, as an alternative to the conventional methods used in the $\mathrm{THz}$ region and optics All measurements, both spectroscopy and polarization control, were made by means of a TDS-THz fibrebased system, with photoconductive antennas (PCA), both for the generation and detection of THz.

Keywords: Terahertz, polarizers, sensing, beam profiling, diffraction. 


\section{RESUMEN}

En el presente trabajo, se investigan algunos aspectos de la interacción de los materiales dieléctricos con ondas de $\mathrm{THz}$. La banda de $\mathrm{THz}$, que incluye las frecuencias de $100 \mathrm{GHz}$ a $30 \mathrm{THz}$, ha despertado un gran interés sobre todo por su baja energía y su buena capacidad de penetración en materiales de uso diario como tejidos y plásticos. Las características peculiares de estas ondas permiten su aplicación en diversos campos tecnológicos, especialmente como herramientas científicas y para la inspección de control de calidad. Para avanzar en la aplicación práctica de la radiación $\mathrm{THz}$, la presente tesis doctoral investigó varios caminos. En primer lugar, se trataron métodos alternativos de bajo coste para la fabricación de componentes pasivos de $\mathrm{THz}$, centrándose en los polarizadores. El interés en el control de la polarización nace de la demanda de un mayor control sobre las características físicas de los haces de THz. Asimismo, se han investigado los polarizadores wire grid flexible basados en el efecto de absorción dicroica. Se han fabricado polarizadores con grafito y Galn24,5 depositados en materiales ordinarios utilizados como sustratos (papel y polímeros como PVA y PVC). Mediante la colaboración con el grupo de investigación chino dirigido por el profesor Liu de la Universidad de Tsinghua, se investigaron procesos de fabricación alternativos. Todos los componentes se simularon mediante un simulador comercial basado en la técnica de integrales finitas FIT (CST Microwave Studio). En segundo lugar, se investigó el potencial de la técnica de fixed delay para la detección rápida de elementos homogéneos y transparentes con posible aplicación en la inspección de calidad industrial. En este esquema, la variación de corriente del haz de $\mathrm{THz}$ en un punto específico está relacionada con la variación de sus parámetros ópticos, por lo que se demostró la capacidad de detectar defectos, así como de estimar sus volúmenes bajo ciertas condiciones. Por último, un enfoque diferente para el beam profile basado en un slit dieléctrico fue evaluada como alternativa a los métodos convencionales utilizados en la región de $\mathrm{THz}$ y la óptica. Todas las mediciones, tanto la espectroscopia como el control de la polarización, se hicieron por medio de un sistema basado en fibra TDS-THz, con antenas fotoconductoras (PCA), tanto para la generación como para la detección de THz.

Palabras clave: Terahertz, polarizadores, sensing, beam profiling, difracción. 


\section{RESUM}

En el present treball, s'investiguen alguns aspectes del paper dels materials dielèctrics quan interactuen amb ones de $\mathrm{THz}$. El bandgap de $\mathrm{THz}$, que cobreix les freqüències de $100 \mathrm{GHz}$ a $30 \mathrm{THz}$, ha despertat un gran interés principalment a causa de la seua baixa energia i la seua bona capacitat de penetració en alguns materials d'ús diari com els teixits i els plàstics. Les característiques peculiars de les ones de terahertz permeten la seua aplicació en diversos camps tecnològics, especialment com a eines científiques i per a la inspecció de control de qualitat. Per a avançar en l'aplicació pràctica de la radiació THz, la present tesi doctoral investiga diversos camins. En primer lloc, es s'han tractat mètodes alternatius de baix cost per a la fabricació de components passius de $\mathrm{THz}$, centrant-se principalment en polaritzadors de THz. L'interés en el control de la polarització es deriva de la demanda d'un major control sobre les característiques físiques dels feixos de THz. Així mateix, s'han investigat els polaritzadors amb reixes de filferro flexible basats en l'efecte d'absorció dicroica. Els polaritzadors s'han fabricat utilitzant grafit i Galn24,5 desplegats en materials ordinaris utilitzats com a substrats (paper i polímers com el PVA i el PVC). Mitjançant una col·laboració empresa amb el grup d'investigació xinés dirigit pel professor Liu de la Universitat de Tsinghua, es van investigar processos de fabricació alternatius. Tots els components es van simular mitjançant un simulador comercial basat en la tècnica d'integrals finites FIT (CST Microwave Studio). En segon lloc, s'ha investigat el potencial de la tècnica de retard fix per a la detecció ràpida d'elements homogenis i transparents amb possible aplicació a la inspecció de qualitat industrial. En aquest esquema, la variació actual del feix de THz en un punt específic està relacionada amb la variació dels seus paràmetres òptics, per la qual cosa es va demostrar la capacitat del mètode per a detectar els buits així com en l'estimació aproximada dels seus volums sota certes condicions. Finalment, un enfocament diferent per al perfil de feix basat en una obertura d'escletxa dielèctrica ha sigut avaluada, com a alternativa als mètodes convencionals utilitzats a la regió de THz i l'òptica.Tots els mesuraments, tant l'espectroscòpia com el control de la polarització, es van fer per mitjà d'un sistema basat en fibra TDS-THz, amb antenes fotoconductores (PCA), tant per a la generació com per a la detecció de THz. Paraules clau: Terahertz, polaritzador, sensado, beam profiling, diffracció 
CONTENTS

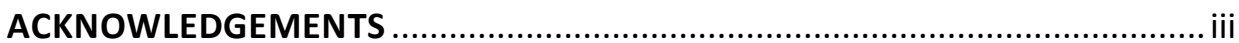

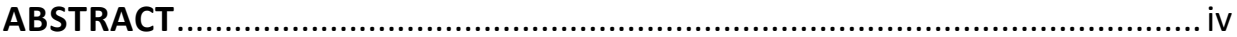

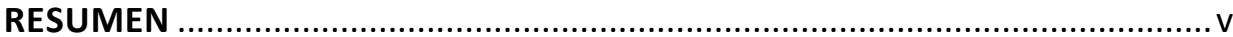

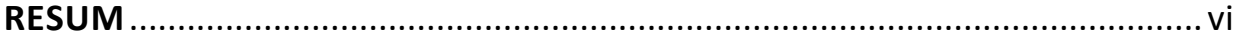

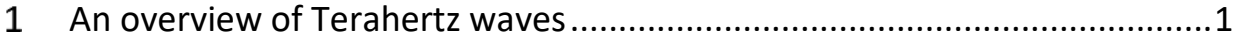

1.1 Terahertz generation and detection .......................................1

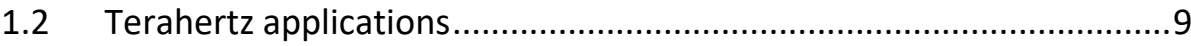

2 Dielectric substrate-based polarizer ................................................. 12

2.1 Polarizer theory ................................................................... 13

2.2 Analysis of the $\mathrm{THz}$ response of a simple periodic graphite-based structure 26

2.2.1 Graphite-based polarizer fabrication ....................................26

2.2.2 Experimental results...................................................... 27

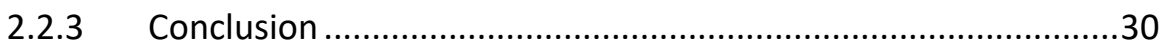

2.3 Flexible Sub-THz Metal Wire Grid Polarizer Based on an EGaln ${ }_{24.5}$ Alloy 31

2.3.1 EGaln 24.5 based polarizer fabrication: drawing .......................31

2.3.2 Experimental results.......................................................33

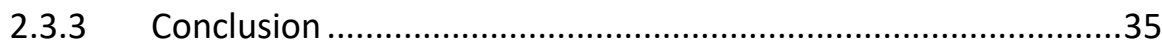

2.4 Printing sub-THz wire grid polarizers using a composite liquid metal ink 37 
2.4.1 EGaln 24.5 based polarizer fabrication: printing.

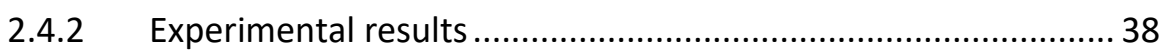

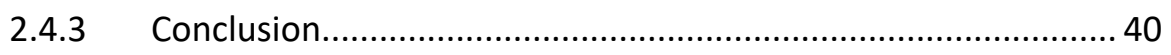

3 Fast quantitative characterization of defects with fixed-delay $\mathrm{THz}$ sensors 43

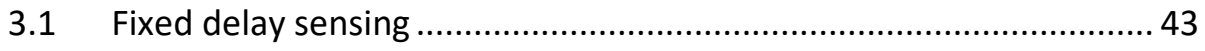

3.2 Principle of operation .................................................................... 45

3.2.1 Performance analysis.......................................................... 47

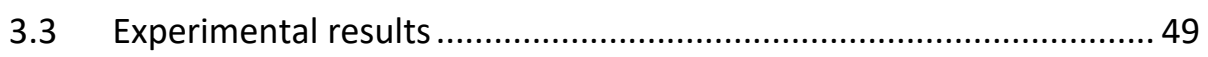

3.3.1 Void detection and characterization .......................................... 50

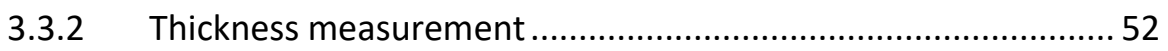

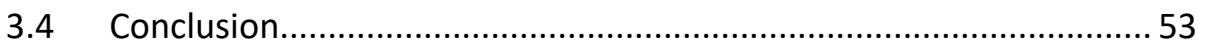

4 Dielectric - based beam profiler ........................................................... 55

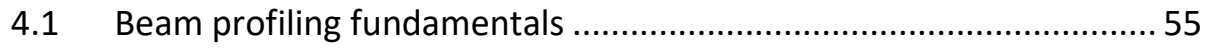

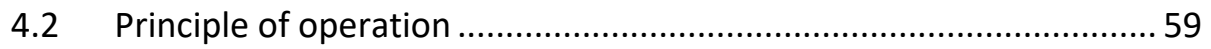

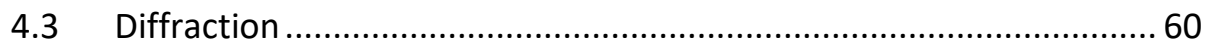

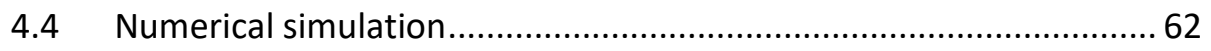

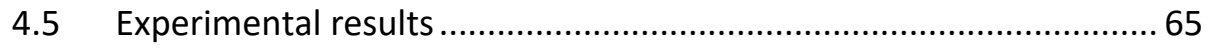

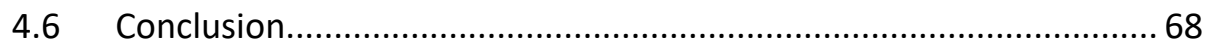

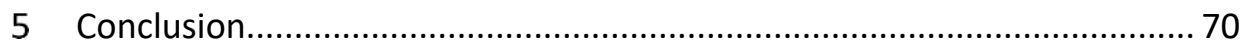

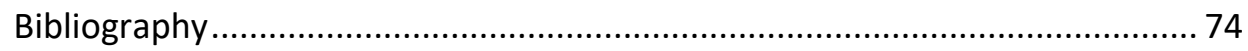

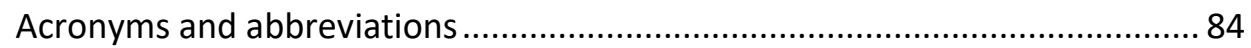

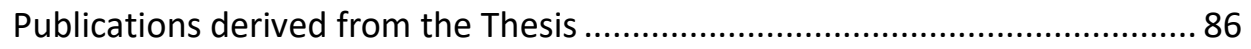

I. Annex - Mathematical study of diffraction on the slit.............................. 87 

he frequency band ranging from 0.1 to $30 \mathrm{THz}$, known as the Terahertz ( $\mathrm{THz}$ ) band or far infrared, and shown in Fig. 1, keeps raising more and more interest among researchers of different disciplines. It is one of the last regions of the electromagnetic spectrum to be exploited and, being between the infrared and microwaves, it has a combination of features from both.

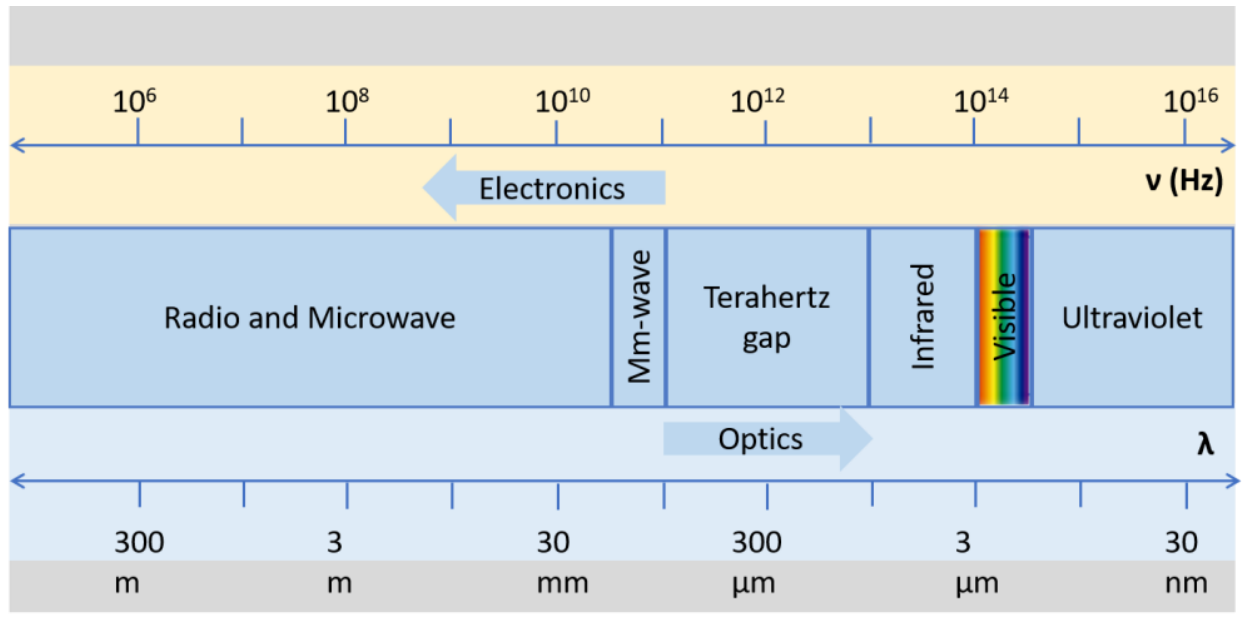

Fig. 1: Sketch of the Terahertz band in the electromagnetic spectrum.

\subsection{Terahertz generation and detection}

Since recently, technology for efficient generation and detection of radiation in this last frontier of the spectrum was basically lacking. Thus, it was dubbed the " $\mathrm{THz}$ gap". At both sides of the gap there were well-established technologies: optoelectronics and electronics. The THz region, falling in between, was not well suited for these approaches and new technology needed to be developed. 
$\mathrm{THz}$ energies are of the order of $4 \mathrm{meV}$, much lower than the electronic state transitions of atoms and molecules used in light sources. Thus, matter properties of popular materials are not adequate to downscale optical sources to emit radiation in the $\mathrm{THz}[1],[2]$. On the other hand, microwave components could be redesigned to operate in this band. However similarly, semiconductors material bandgaps exceed the $\mathrm{THz}$ energies, so that using solid-state devices for $\mathrm{THz}$ generation is still challenging.

Despite these issues, optical and electronic technologies have made remarkable progress to reduce this technology gap in the last three decades. Researchers came up with several different approaches to generate and detect THz waves, as detailed in the next paragraphs.

The main methods for the generation of broadband and CW Terahertz are:

- Non-linear media, as in optical rectification, Difference Frequency Generation (DFG), and frequency multiplication of microwaves.

- Accelerated electrons, i.e. from photocurrent in a semiconductor or from free electrons in vacuum.

- Population-inversion of two-level quantum system, such as Far-IR gas lasers, solid state P-type Germanium lasers and Quantum Cascade Lasers $(\mathrm{QCL})$.

Processes belonging to the first category obtain terahertz waves by stimulating second order nonlinear optical phenomena with a subpicosend response in the excited non-linear medium [3], [4]. Optical rectification generates DC or low frequency polarization when a strong laser beam propagates through a crystal [2], through the electrooptic effect (Pockel's effect). While, in DFG, two continuous optical beams are used to generate a CW THz wave, by exploiting the same secondorder nonlinearities of electro-optical (EO) crystals. The regular alternation of the polarization state of the medium at the difference frequency induces the generation of a $\mathrm{THz}$ wave, with a frequency equal to the difference between the two lasers sources. The crucial aspect that has to be considered when applying this technology is to ensure phase matching in the material. Finally, nonlinearity is also employed in electronic devices such as non-linear diodes, where it is used to convert low frequency microwave signals from solid - state $\mathrm{THz}$ sources to their upshifted harmonics. 
A completely different approach for the generation of these waves relies on the field emitted by accelerated charges. Transient photoconductive switches, excited by a broadband femtosecond pulsed laser, generate a time-varying photocurrent proportional to the intensity of the impinging optical pump. Thus, ultrashort optical pulses are converted into a few cycles of a $\mathrm{THz}$ wave. A similar procedure is followed with photomixing to generate $\mathrm{CW}$ THz waves, in which two sources at different frequencies are mixed to form an optical beat [1]. An optical beam impinges the gap formed by two metal electrodes deposited on a semiconductor material with proper carriers features. The parameters of the involved elements must be considered to fully understand the generations and detection $\mathrm{THz}$ process [5]. Therefore, the semiconductor material of the antenna, its geometry and the optical pulse are briefly discussed in the following.

When choosing the optimum semiconductor material, four elements should be considered:

- Carrier lifetime: it governs the first half cycle of the T-ray, since it determines the time needed to close the switch in the receiver. This parameter should be shorter than the $\mathrm{THz}$ pulse duration, i.e. of the order of sub-picosecond scale. Such short lives are obtained through a strong doping of the material. However, it must be considered that too many traps unduly affect the mobility of the charges, so it is necessary to ensure the balance between these two parameters.

- Carrier mobility: it should be high to lead to fast rise times.

- Breakdown voltage: it should be as high as possible, without damages the material itself. However, it is balanced by the maximum radiation power and the external bias.

- Dark resistivity: it should be high to ensure that almost no current flow is present without excitation (typical values are of the order of $M \Omega$ ).

The most commonly used semiconductor materials that fulfil all these requirements are low-temperature-grown gallium arsenide (LT-GaAs) for excitation with $800 \mathrm{~nm}$ and InGaAs-InAlAs multilayer heterostructures for $1550 \mathrm{~nm}$. New materials have recently been developed to enhance peak dynamic range [6]. The antenna geometry determines the power and bandwidth of the THz beam. The most popular structures are stripline, dipole, and offset dipole electrodes. The antenna's gap is of the order of the optical pulse spot, i.e. less than tens of microns. 
Finally, some consideration should be made about the role of the optical pulse:

- Its duration governs the first half of the THz single cycle, because it is responsible of the antenna time response, plus it ensures a broad spectral bandwidth

- The power should be calibrated as to counterbalance the screening effect generated by the free charge flow, that induces $\mathrm{THz}$ pulse saturation at high optical pumps.

- The wavelength should be comparable with the antenna gap width.

Broadband coherent $\mathrm{THz}$ radiation can also be generated from transient electron relativistic acceleration, achieved both by smashing a set of electrons into a metal target or by forcing them into a circular motion by magnetic fields [1]. Backward Wave Oscillators (BWOs) and Free-Electron Lasers (FELs) share the same $\mathrm{CW} \mathrm{THz}$ generation mechanism, obtained with periodic electrons accelerations. BWOs are laboratory size equipment, consisting in a vacuum tube where the undulation of an electron beam is generated by a metal grating. On the contrary, FELs are large facilities since they are small-scale electron accelerators, which obtain the same effect with a magnetic array that varies according to distance which represents a severe drawback that overshadows its valuable performance [7].

Finally, a third type mechanism makes use of lasing processes whose media transition frequencies fall into the $\mathrm{THz}$ spectral region. For example, Far-IR lasers rely on molecular rotational energy level. P-type Germanium lasers achieve $\mathrm{THz}$ broadband radiation by emission of LO-phonons. Another approach is based on QCL. These are semiconductor lasers in which light emission is enabled by means of intersubband transitions in a repeated stack of semiconductor multiple quantum well heterostructures [1]. In general all these solutions require cryogenic cooling [7], but improvements have been made in the last years both in terms of operating frequency and conditions [8].

These are generation methods which need to be matched with a detector. Methods for $\mathrm{THz}$ detection [9] can be primarily divided into incoherent or coherent detection, depending on if they measure both amplitude and phase of the waves or not. Fig. 2 shows the main detection scheme depending on their operational principle. 


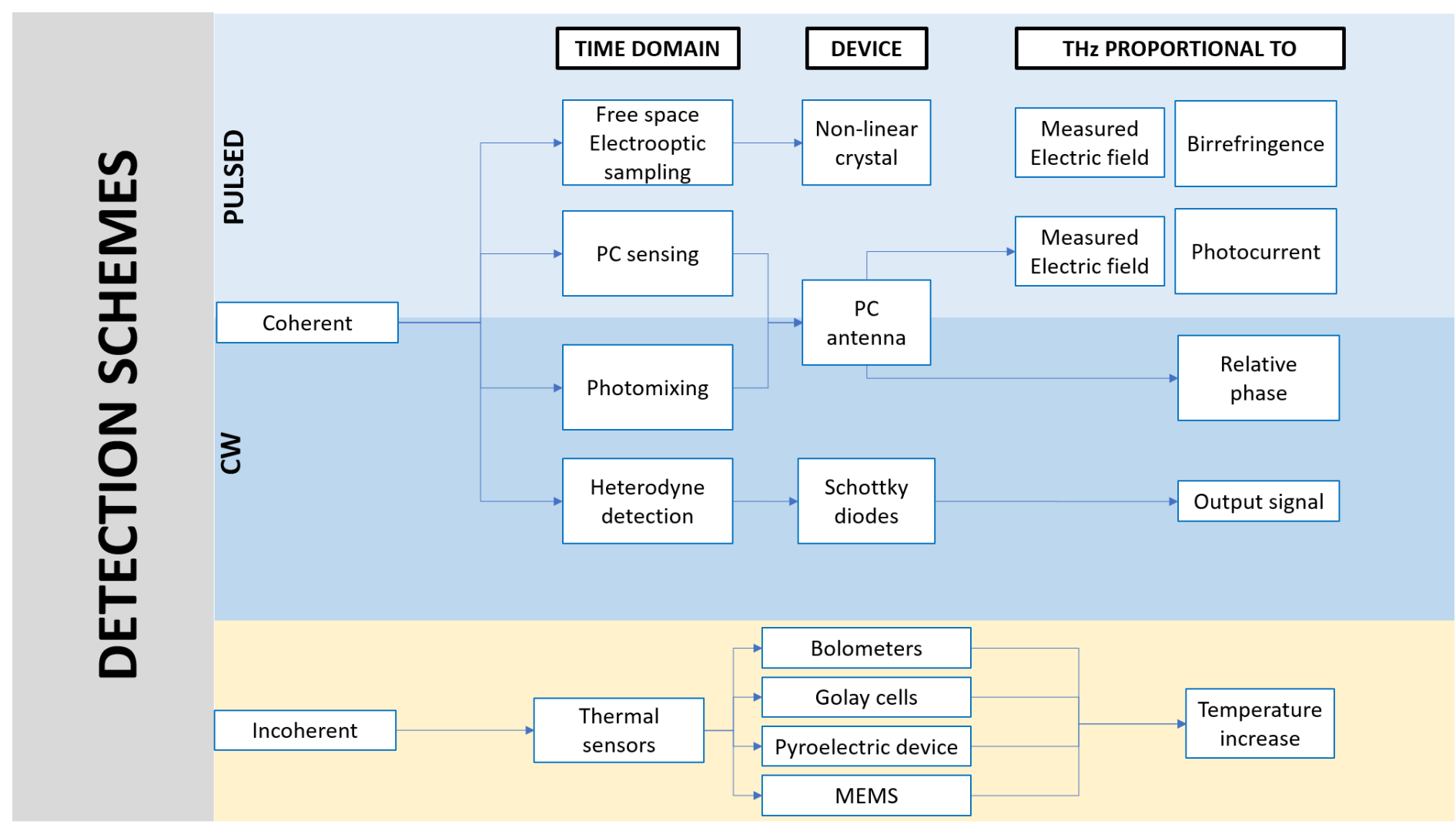

Fig. 2: Main detection scheme depending on their operational principle. 
Among coherent detection methods, both pulsed and CW versions are available. To the first group belongs both free space electro optic sampling [10], [11], [12] and photoconductive (PC) sensing, in which the $\mathrm{THz}$ electric field is proportional respectively to the birefringence effect generated in a non-linear crystal and to the transient photocurrent induced in a PC switch, also known as photoconductive antenna. These components [13] are based on the inducing of a rapid time dependent change of a parameter of a material. The creation of charge carriers causes the rapid modification of the conductivity of a fast-response semiconductor material. The temporal derivative of the induced photocurrent is directly proportional to the amplitude of $\mathrm{THz}$ electromagnetic field. Electronic heterodyne schemes rely on the ability of Schottky diodes of measuring the output signal that is proportional to the $\mathrm{THz}$ amplitude.

Another approach is based on incoherent detection schemes. Thermal sensors [14] record the temperature increase, a direct consequence of the absorption of THz. These include bolometers [15], Golay cells [1] and pyroelectric devices [1]. Although incoherent detectors can detect a wide range of frequencies, they are intrinsically slow, and the most sensitive ones require cooling to avoid the influence of background thermal radiation.

The described photoconductive and nonlinear THz emitters are well suited for spectroscopic measurements when combined with a similar $\mathrm{THz}$ detector [16]. Among these, broadband $\mathrm{THz}$ generation and detection is probably the most widespread tool to investigate transient phenomena and to implement $\mathrm{THz}$ sensing equipment. These provide coherent phase information to simultaneously determine the absorption and dispersion of the sample, without using KramersKronig relations as in near-infrared spectroscopy [1]. In particular, pump - probe schemes, as the one illustrated in Fig. 3, are employed. It will be the basic experimental setup to be used through the Thesis ${ }^{1}$.

\footnotetext{
${ }^{1}$ The basic experimental setup used through the Thesis, and shown in Fig. 3 is an all-fiber THz-TDS system based on a 100 fs fiber laser (by Toptica AG), two photoconductive antennas (InGaAs/InAlAs multilayer heterostructures by Menlo Systems) with hyperhemispherical attached lenses and a step-wise optical delay line. Commercial components and control software were developed in-house. A modulation signal is set at $30 \mathrm{kHz}$ frequency and detection is performed with a lock-in amplifier (Stanford Research). A set of four TPX lenses (biconvex - L2, L3 and plano-convex - L1, L4) are used to collimate and focus the THz beam. The optical pulse travels along a fiber path that consists of Single Mode Fibers (SMF ) and Dispersion Compensating Fibers (DCF).
} 


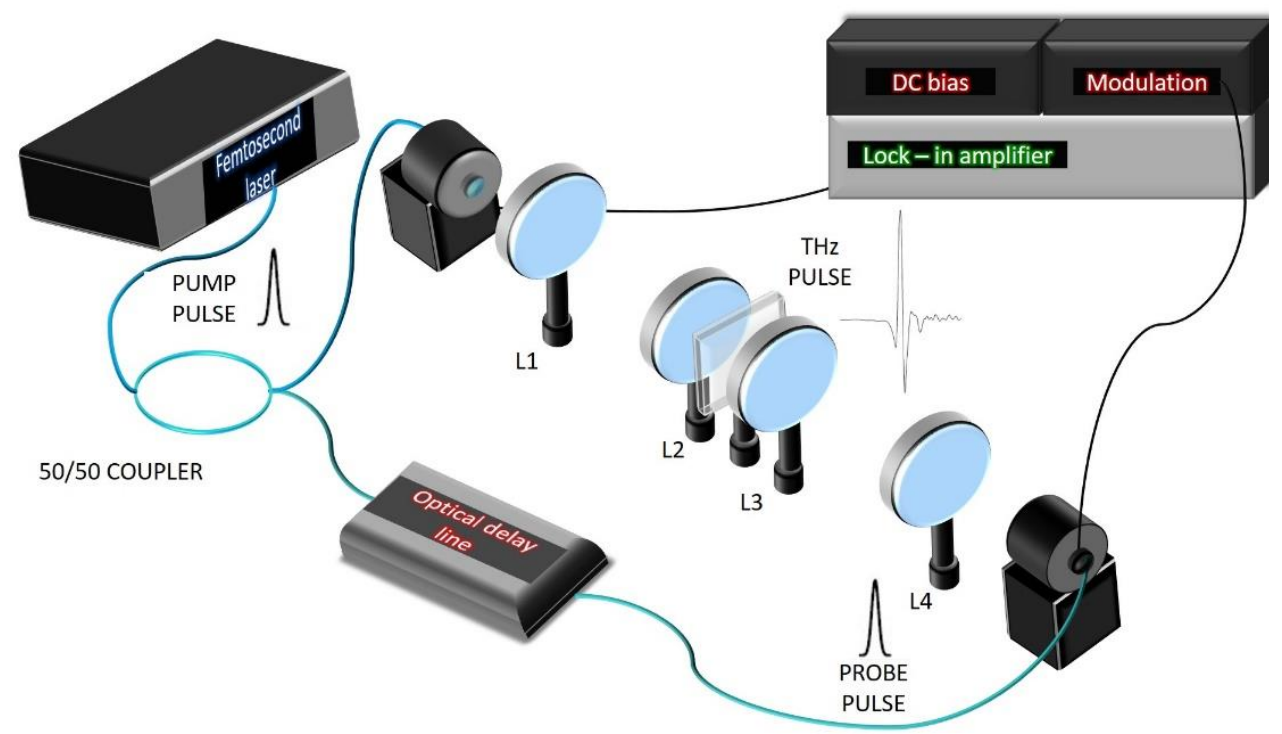

(a)

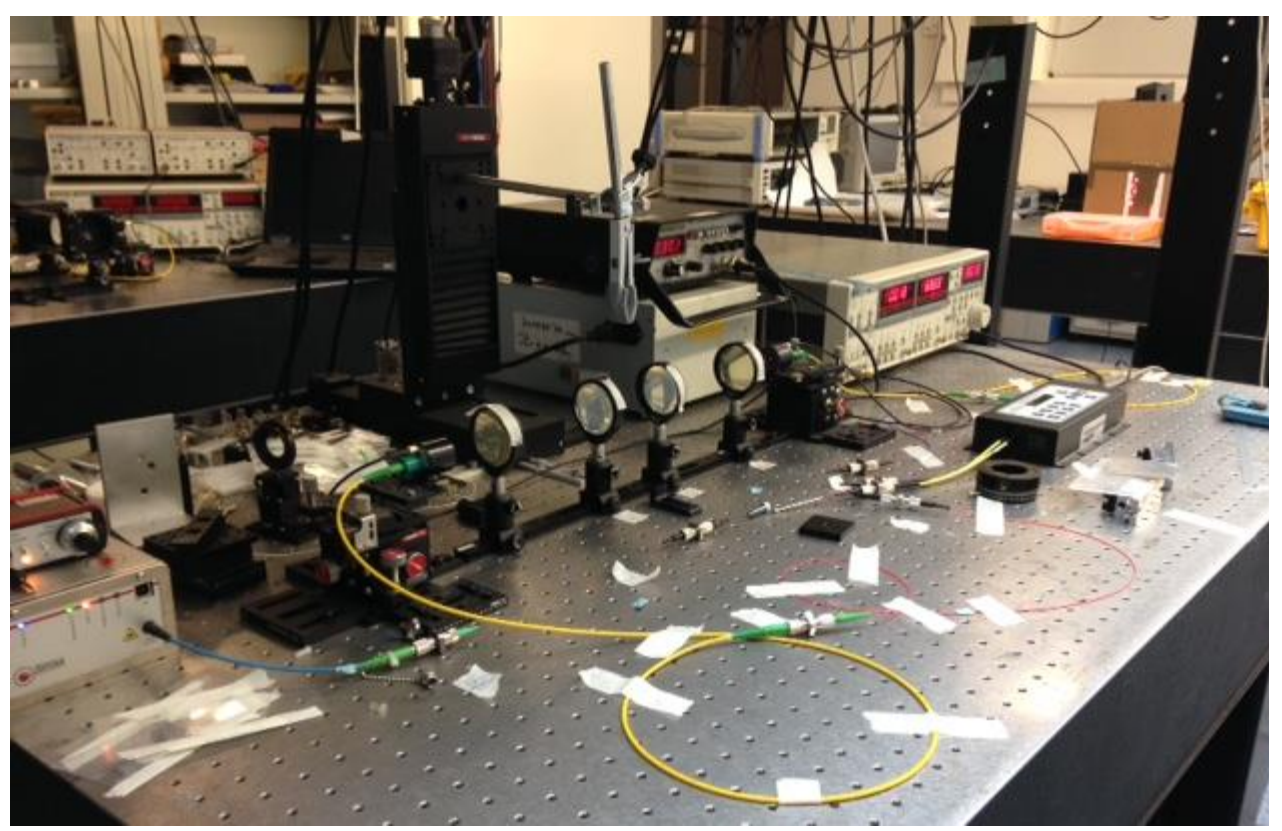

(b)

Fig. 3: (a) Pump-probe detection scheme and (b) real measurement set up used for the measurements of the present work.

In this broadband $\mathrm{THz}$ pump-probe setups, the $\mathrm{THz}$ emitter and $\mathrm{THz}$ detector are excited with fractions of the same synchronized train of femtosecond pulses, named pump and probe beam, respectively. The adjustment of the relative time delay is obtained by means of translational stages that changes the beam path. These optical delay lines can be both mechanical (which include point to point [6], [17] and voice-coil ones [18]) or implemented through opto-electronic techniques such as ECOPS or ASOPS [19], [20]. 
The most general pump-probe scheme is named THz time-domain spectroscopy, or, in short, THz-TDS [16]. It can be based both on non-linear or photoconductive generation and detection, being the latter of more interest for the scope of the present work. The direct measurement of the field allows for the simultaneous measurement of both intensity and phase of the $\mathrm{THz}$ electric field. In addition, photoconductive antennas can be pigtailed. It led to robust all-fibre based instruments with compelling features for industrial quality control [21] and even centralized architectures [22].

The elements needed to implement a photoconductive-based THz-TDS system include in such schemes are photoconductive switches, a DC external bias, a current amplifier and a femtosecond laser as the optical pump-probe source. A brief overview of the roles of these elements is given in next paragraphs.

A photoconductive (PC) switch is composed of two metal electrodes, the antenna structure, deposited on a semiconductor [1], [23]. The ultra-short optical pulse impinges impulsively into the semiconductor of the antenna gap, stimulating the creation of free carriers, allowed to overcome the energy bandgap and enter the conduction band. The increase in the number of free carriers in this band enables the rise of its photoconductivity. An external DC bias is applied to the electrodes to accelerate the charges: the photocurrent rises, while the charge density in the conduction band decreases, due to the semiconductor defects, which capture them. The resulting impulse of current gives rise to a single cycle $\mathrm{THz}$ electromagnetic field, i.e. a transient on a sub picosecond scale. The signal power depends on the external DC bias and on the power of the optical pump. The generation process can be analysed using the Lorenz-Drude model since the source size is much smaller than the $\mathrm{THz}$ wavelength generated (10 $\mu \mathrm{m}$ optical beam spot versus $300 \mu \mathrm{m} @ 1$ THz) [5], [24]. Therefore, the antenna can be approximated to a Hertzian dipole. Moreover, research in the field of photoconductive antennas is still of great interest to enhance their performance, mainly through the development of new dopings [6] but also the use of nanostructures is being pursued [25].

The detection mechanism [26] follows the same ground principle, yet in this case the optical impulse closes the switch and thus the THz pulse is sampled. On a subpicosecond scale, the semiconductor changes from being almost insulating to being conductive when the probe pulse arrives and then again insulating when the switch is reopened and no current flows. A high-sensitivity current amplifier is crucial to 
detect the created current flows through the antenna gap, because of its sub picosecond resolution. Lenses are required to enhance the coupling of the generated $\mathrm{THz}$ from the semiconductor material to free space [27] and either reflective or transmissive optics configurations can be designed, so that the $\mathrm{THz}$ beam can travel towards the receiver.

In the present work, an in-house developed all fibre-coupled system has been used to perform the experiments. It is based on Erbium-doped fibre lasers in the $1550 \mathrm{~nm}$ band, i.e. the telecom band. Optical fibre technology is of great help in THz-TDS, by offering low-losses and implementations with high stability [28], [22].

\subsection{Terahertz applications}

The aforementioned technologies reviewed in the section have allowed the exploitation of THz waves for plenty of sectors. Their capability of detecting both amplitude and phase of coherent $\mathrm{THz}$ radiation, makes Terahertz technology extraordinarily appealing for both spectroscopy and imaging applications in a wide range of different fields [29], of which some examples are given below.

$\mathrm{THz}$ technology has opened a new window to understand different processes. It has been applied in the fields of chemistry [30], [31], [32] and biology [33], [34], [35], bioengineering and atmospheric and astronomical spectroscopy (remote sensing) [36]. The capability of $\mathrm{THz}$ radiation to penetrate on nonpolar dielectrics attracted considerable interest for security applications. Although the initial expectations of exploiting the whole $\mathrm{THz}$ range for security screening were not fulfilled, it is still used is some niche applications, such as mail screening [37].

Finally, another field where $\mathrm{THz}$ signals can make a difference is in sensing and quality. Terahertz based sensors offer interesting features for quality inspection [16], especially when it relies on all-fibre photoconductive-based technology due to its capability to combine compact and robust deployment with good dynamic range [28], [22]. Waves in this band offer submillimetre spatial resolution, being more appealing than microwave-based systems. Furthermore, they are non-ionizing and harmless for human beings. Additionally, they can detect both defects on the surface and within non-polar dielectrics, even providing tomographic images [1]. $\mathrm{THz}$ waves are strongly absorbed by water and humidity (water vapor has absorption lines that fall into the $\mathrm{THz}$ frequency range), and they show good optical penetration for dielectric materials, such as polymers, paper, cardboard and some fabrics, among many others. As an example, paper contains high quantities of 
water, so THz are mostly absorbed. Nonetheless, if the sheet of paper is sufficiently thin, it is almost transparent. Thus, $\mathrm{THz}$ radiation can be used to extract parameters such as its composition or water content.

These features make $\mathrm{THz}$ sensing very attractive for quality control of plastics, ceramics and compound materials, which lack practical tools for the nondestructive inline monitoring of several key parameters. As a result, great efforts are being made to bring $\mathrm{THz}$ technology out of the laboratories to penetrate industrial environments, pushing the boundaries in fields such as packaging, automotive, aerospace or food sector.

Thus, $\mathrm{THz}$ are now considered as a valuable tool for monitoring compounds [38], [39], [40], [41], [42], [43] and polymeric compounding processes [21], [44], [45], [46], [47] as well as paintmeters [48], [49], [50], [51] in which even the thickness of each layer can be measured individually, by recording the time of flight signals. $\mathrm{THz}$ signals are also useful for non-destructive inspection of food and agri-food products for foreign bodies identification, both hard and soft [52], [53], detecting counterfeiting in integrated circuits [54].

Quality control can be done through THz imaging [55], [56], [57] for which, recently, several techniques [58], [59] have been investigated to enhance resolution. On the other hand, in many industries, $\mathrm{THz}$ imaging may not be necessary and the easier detection of changes in the $\mathrm{THz}$ trace can reveal defects such as dents, delamination, voids, protrusions, etc. in relation to the reference sample [60]. In particular, undesired voids have a detrimental effect on the final material properties, leading to the shortening of the product lifecycle. 
1. An overview of Terahertz waves 


\section{Dielectric substrate-based polarizer}

$\mathrm{n}$ the last decades many efforts have been devoted to push THz technology from scientific towards industrial applications. To contribute towards this goal, new systems concepts are being developed, such as fibre based $\mathrm{THz}$ systems [28] and centralized architectures [22] for robustness and cost reduction respectively. In parallel, new components are also being researched from a wide range of areas. It includes new compact fibre-based optical sources, new photoconductive antennas [6] and also a plethora of passive components such as filters, lenses, beam splitters, absorbers, wave plates and polarizers.

Although $\mathrm{THz}$ passive components bear a close similarity with their optics counterparts, the longer wavelength of the $\mathrm{THz}$ band allows for alternative fabrication methods with larger tolerances to achieve cost-effective devices. For example, this feature of $\mathrm{THz}$ waves has been extensively exploited to implement low-cost beam splitters [61], [62], [63], achromatic wave plates [64], [65], periodic structures, such as polarizers [66], [67], metamaterials [68] and diffractive paper lens [69].

This chapter is devoted to investigating the feasibility of this research line but for the implementation of low-cost THz polarizers. THz polarimetry is a powerful tool to extract additional information from samples [70], [18], [71]. Polarization sensitive measurements are very valuable in $\mathrm{THz}$ sensing, for example, through $\mathrm{THz}$ polarimetry [72], [73], [74] and, in particular in the chemical and pharmaceutical industries [75]. They could also rapidly detect the orientation of a passive 
component polarization - dependent to ensure its use in the optimal operating condition, without affecting the experiment result.

\subsection{Polarizer theory}

A polarizer can be defined as a passive device that can separate a desired state of polarization (SOP) at its output. The ultimate polarization state of a wave after crossing a complex optical system can be described in terms of the mathematical formalism based on vectors and matrices. Polarization states of the wave are described in terms of vectors, whereas matrices represent the optical elements which transforms the input wave. In this way, each optical component has a specific representation in the mathematical formalism. Depending on whether the wave is randomly polarized, partial polarized or fully polarized, different mathematical parametrization should be used. Thus, Jones vectors and $2 \times 2$ Jones matrices are needed to represent fully polarized light. On the contrary, Stokes vectors, which contain both the unpolarized and polarized part of the light, and 4x4 Müller matrices are required to find the resulting polarization of an emerging wave after crossing an optical system. The resulting output could be either elliptical, circular or linear, the former being the most general case, and specific elements are necessary to manipulate these states to make them fit the experimental needs.

Polarizers are the most suitable tools for this purpose, since they are able to filter some defined polarization states, discarding the unwanted waves. Although this goal is achieved by mean of different physical mechanisms, all polarizers take advantage of a specific anisotropic response of a structure or material when it is impinged by polarized (or unpolarized) light (see Fig. 4).

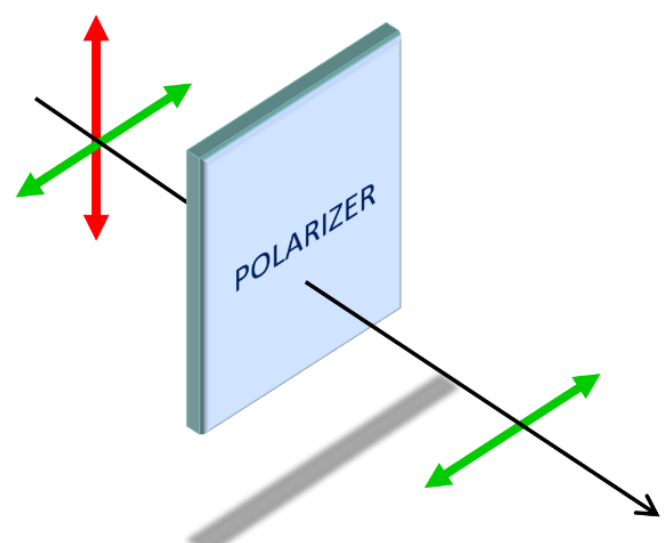

Fig. 4: Sketch of the polarizer functioning. 
Linear polarizers are of main interest for this work and they will be reviewed in detail. Different linear polarizer types have been investigated, each one relying on a separate physical mechanism. Fig. 5 reviews the main strategies to implement $\mathrm{THz}$ polarizers. As It can be seen, they mainly rely on dichroism, reflection and birefringence mechanism. 


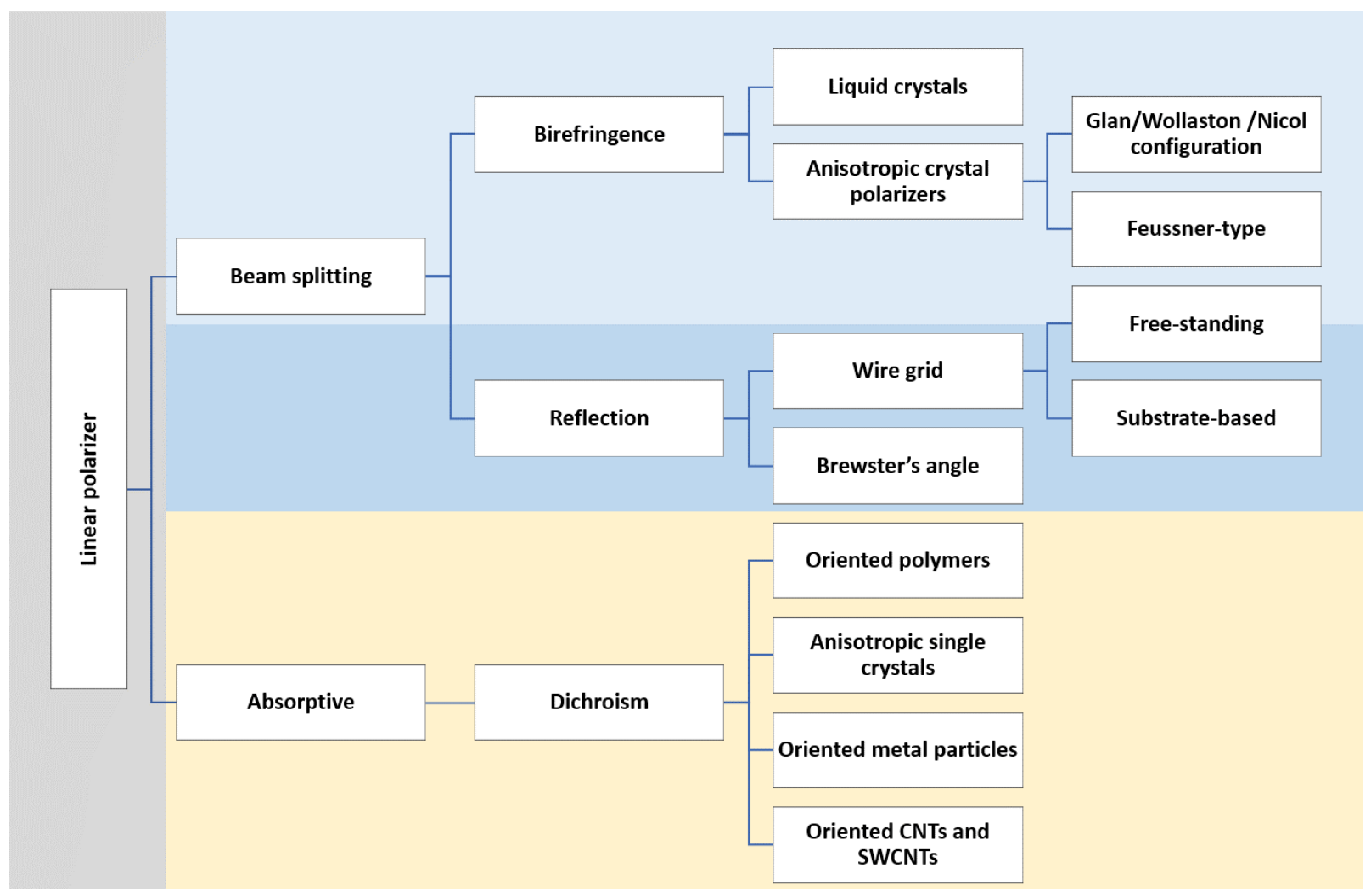

Fig. 5: Classification of Terahertz linear polarizers. 
Depending on the relying physical mechanism, terminology used to describe the desired and unwanted polarization state is different.

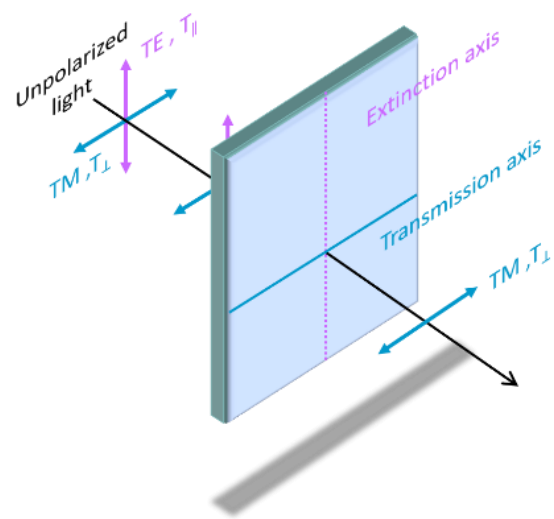

(a)

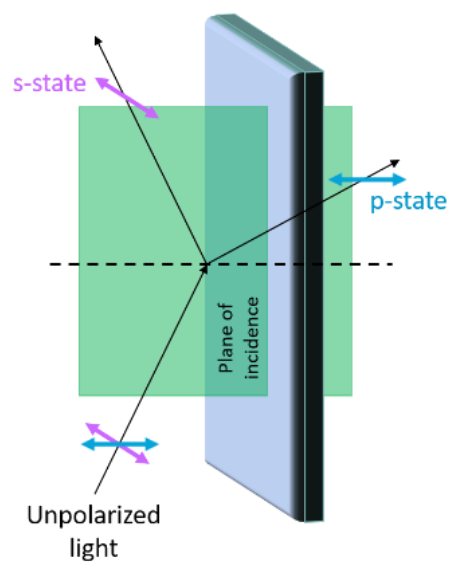

(b)

Fig. 6: Sketches of the nomenclature used to define the desired and unwanted polarization state in linear polarizer.

Thus, for dichroic polarizers, the polarization state perpendicular to the extinction axis (that is, parallel to the transmission axis), named $T_{\perp}$ is the passing wave, whereas the parallel component $T_{\|}$is the absorbed one. Generally speaking, a wire grid polarizer transmits TM-polarized (transverse magnetic) light with electric field perpendicular to the wires while it reflects TE polarization (transverse electric). For reflection-based linear polarizers, $\mathrm{p}$ - and s- states are introduced. The $p$ - state lies in the plane of incidence, formed by the propagation direction of the impinging wave and the normal to the incidence surface. On the contrary, the sstate is perpendicular to the plane of incidence (see Fig. 6).

There are several parameters that serve as indicators to evaluate the performance of a polarizer. All of them are described in terms of their transmission and extinction axis, perpendicular to each other.

Linear polarizers must follow Malus's law, which states that the irradiance that crosses the linear polarizer is proportional to the angle between the initial light polarization direction and the transmission axis: $I(\theta)=I(0)(\cos \theta)^{2}$, being $I(0)$ the irradiance impinging on the polarizer. This polarizing behaviour is usually tested in an analyser configuration in which, besides the polarizer under test, a second one, called the analyser is placed in the beam path. In this case, the angle $\theta$ is the one formed by the transmission axis of the two polarizers and the maximum transmission is achieved when they are parallel $\left(\theta=0^{\circ}\right)$. Other common parameters used to evaluate the goodness of a polarizer are: 
- The Extinction ratio (ER), that refers to the ability to discriminate between the desired $\left(T_{\perp}\right)$ and the unwanted polarization state $\left(T_{\|}\right)$in a linearly polarised beam $\left(E R=10 \log _{10} \frac{T_{\perp}}{T_{\|}}\right)$.

- The Degree of Polarization (DOP), that defines how much polarized is light, ranging from 1 (or 100\%) for a fully polarized electromagnetic wave or 0 to an unpolarized one.

- Insertion Loss (IL), mostly due to substrate or polymer, that measures how much radiation is lost, due to multiple effects, when it travels across the device $\left(I L=10 \log _{10} T_{\perp}\right)$.

The choice of whether use one polarizer instead of another is strongly related to the experiment requirements, as well as the needed performance. A briefly discussion on the main features of the existing polarizing devices is provided next.

In absorptive polarizers, dichroism plays the major role in the polarization selection. This polarizer type is based on the property of anisotropic absorption of the polarization state parallel to the extinction axis, phenomenon that is induced by different materials or structures. Typically, the selective absorption is obtained by means of anisotropic single crystals. Their crystalline structure determines a specific direction, called the principal optical axis, that marks the crystal behaviour: the polarization state perpendicular to the optical axis will be strongly absorbed. These properties are wavelength dependent and this limits their wide application. Similar absorptive structures are made of oriented polymers.

Considerable efforts have been devoted to the implementation of absorptive polarizers based on periodic grid structures made of Carbon Nanotubes (CNTs). The anisotropic properties of CNTs derived from their large aspect ratio (the height to width ratio) and small diameters so that they exhibited strong linear dichroism and consequent strong absorption. Single, double and triple layers of SWCNT (Single Walled Carbon Nanotube) on substrate films have been studied [76], [77] as well as thick multiwalled carbon nanotubes (MWCNTs) sheets [78]. A similar approach is based on aligned metal particles, for example, Ni nanoparticles in polyvinyl alcohol (PVA) with an hybridize structure [79]. Absorptive polarizers benefit from 3D printing techniques that allow the fabrication of conductive optical components, as they enable rapid fabrication of arbitrary elements with nanocomposite-based microstructures. Although printing resolution and dispersion of nanomaterials in the matrix is still challenging, direct deposition of intrinsically conductive polymer- 
based 3D microstructures will promote their straightforward and versatile application [80].

Beam splitting polarizers, both transmissive and reflective are presented in the next paragraphs. The first type relies on the birefringence mechanism in which the ordinary and extraordinary rays are directed along different paths, as to suppress the unwanted polarization and transmit the desired component. In the latter, high reflection coefficient governs the polarizer response depending on its structure.

Many crystalline substances belong also to this category, as long as their optically anisotropy translates into an anisotropy in the refractive index, called birefringence. These materials can work as polarizers, as long as they separate the ordinary from the extraordinary ray, depending on the refractive index experienced by each one. For example, calcite is widely employed with this purpose in the visible and ultraviolet (UV) range where it enables high performance, whereas, other anisotropic materials such as $\mathrm{AgGaS}_{2}$, are required at longer wavelengths [81]. In general, it is still harsh to design birefringent polarizer for the THz frequencies, since it is difficult to find transparent birefringent substances in this band. A common solution at these frequencies consists on stacking together oriented birefringence prisms in specific configuration, being the most common the Glan - Thompson, Wollaston (two calcite or quartz triangular again stuck together) and Nicol ones. However, these polarizer structures still fail to meet the high extinction ratio and the broad wavelength coverage that is of utmost importance for the $\mathrm{THz}$. Li et al. 2010 [82] tried to overcome this last shortcoming by designing a compact $\mathrm{THz}$ polarizing beam splitter based on a periodic bilayer structure, which is highly reflective only for TE polarization and highly transmitting for TM state. For this purpose, a multilayer structure was sandwiched between two infrared prisms with angle $\theta$. By properly choosing the incident angle of the beam, the TM polarization was not reflected by the interface between the two high and low refractive indexes and the foreseen separation was accomplished. This was a kind of Feussner-type polarizer that consists in the insertion of an anisotropic media between two isotropic prisms as to separate the two linear polarization components, as they experimented two different refractive indexes. Martínez-Antón et al. 2002 [83] used a stretched polyethylene terephthalate (PET) film inserted into two prisms made of glass. Hsieh et al. [84] also worked on a similar structure, proposing a novel design based on the birefringence and transparency of nematic liquid crystals (NLCs) in the THz band. A rectangular parallelepiped of fused silica was engraved, 
filled with NLCs and finally sealed with Teflon. Magnets were also included to ensure the perfect alignment of the crystals. These structures are not widely commercially available, probably due to their low environmental resistance or the fabrication difficulties.

Finally, there are reflection-based polarizers which probably are the most common in laboratory and industrial applications. These include Brewster and wire grid models.

Brewster polarizers can be included both in transmissive and reflection type, based on their operation principle. The device makes use of the different reflection and transmission coefficients of the orthogonal polarization states at an interface between two materials. The system is designed so that the incident wave impinges the surface at the Brewster's angle as to entirely refracts the p-component. It can be shown [85] that a cost-effective Brewster polarizer with low insertion loss (less than $1 \%$ ) and high extinction ratio (more than $6 \times 10^{3}$ ) can be achieved, by using four inexpensive silicon wafers stuck together and arranged at Brewster's angle, with results comparable to those achieved by wire grid polarizers.

Wire grid polarizers (WGPs) are definitely the most common devices used for the purpose of separating two polarization states in the terahertz frequency range. WGPs, shown in Fig. 7, are made of a periodic subwavelength distribution of parallel conductive wires either free-standing or on a substrate. The underlying mechanisms could involve both the reflection [1] and absorption [86] of the polarization state that is parallel to the wire orientation, the extinction axis, whereas the other one passes unaltered. In particular, a perfect-conductor wiregrid transmits TM-polarized (transverse magnetic) light with electric field perpendicular to the wires while it reflects TE polarization (transverse electric) with electric field parallel to the wires [87].

The key geometrical parameters that mainly govern the final quality of the polarization output are:

- the width of the metal wire

- the thickness of the metal layer

- the period or pitch of the grating (intended as the centre - to - centre distance between two adjacent wires or the sum of the wire width and the spacing).

- The fill factor, that is the wire to pitch ratio 
- Aspect ratio (mostly in the case of metal gratings), that is the height to width ratio.

Better performances are generally achieved in the $\mathrm{THz}$ range by grids with small periods and large wires, because of the high reflection degree of the unwanted component. However, it is worth noting that reducing the pitch size will improve transmission and extinction performance at the cost of increased fabrication complexity.

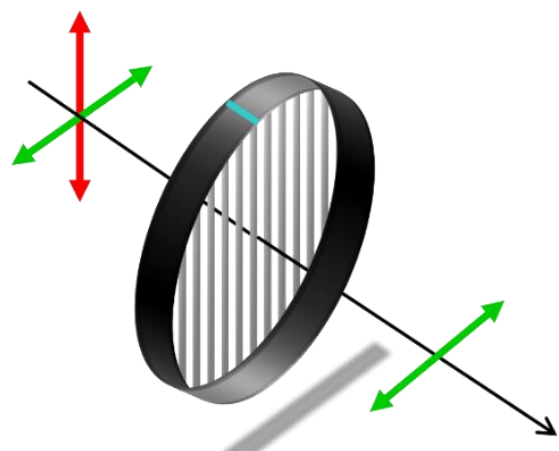

Fig. 7: Sketch of wire grid polarizer.

Free standing grids are those WGPs where there is no substrate, i.e. the wires are suspended on air. These achieve high extinction ratio, thanks to their low losses. However, they are generally expensive since the fabrication requires a rather sophisticated coil - winding process that make them hard to manufacture, in addition to the need of specific materials. The wires are usually made of tungsten since it has the required high tensile strength, a crucial requirement since it must be fixed on a rigid frame. In addition, the overall polarizer is fragile, metals suffer of inevitable oxidation and are susceptible to cracks. Finally, tiny spacings between wires are hard to fabricate when operation at very high frequencies in the $\mathrm{THz}$ is needed, which has a detrimental effect.

Substrate-based grids. Free-standing grids have several shortcomings and limitations in handling and robustness. To gain in robustness, the alternative is to use a substrate. Fig. 8 summarizes the main advantages and drawbacks of such structures. 


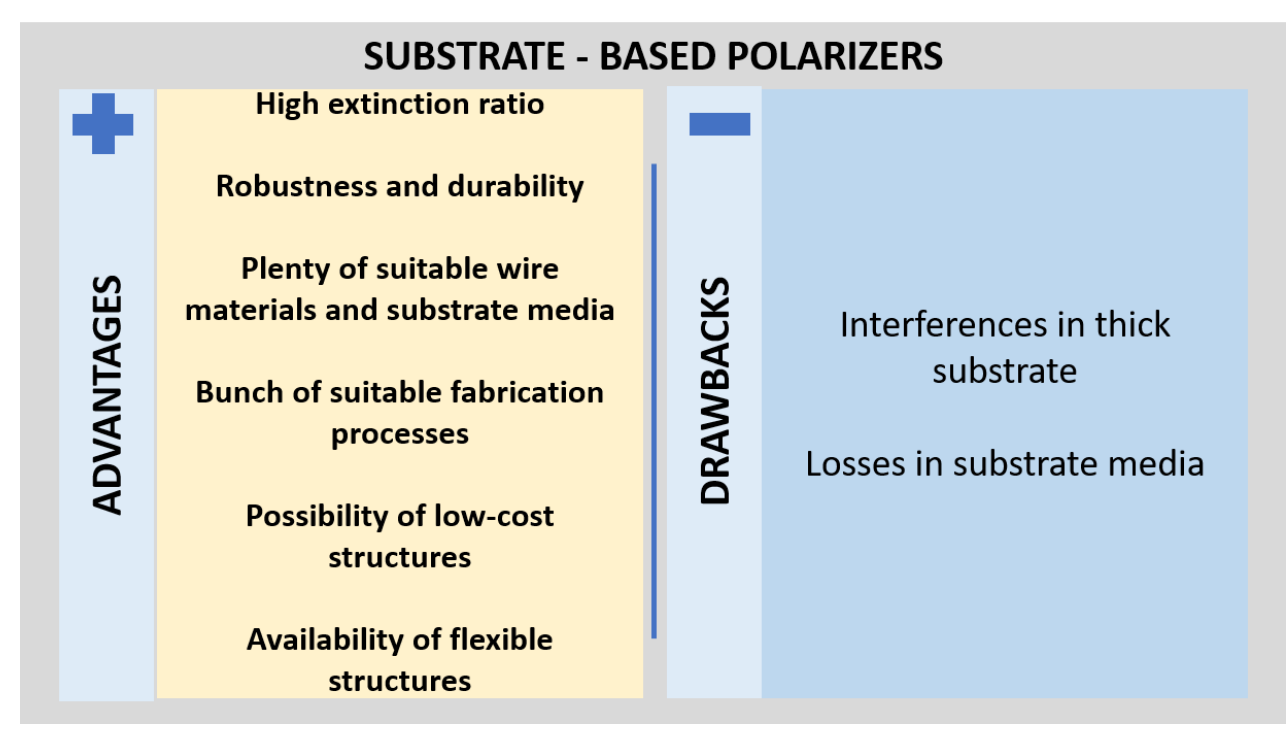

Fig. 8: Main advantages and drawbacks of substrate - based polarizers.

Substrates need to have low - loss and so plenty of materials transparent and semi-transparent to $\mathrm{THz}$ radiation have been examined. Among rigid substrates, the most popular is probably silicon wafers. It is widely employed since it is the standard substrate in electronic and photonic microfabrication and thus it is relatively cheap and compatible with high resolution deposition techniques. Besides, it has appealing attributes such as sufficient chemical stability and mechanical strength. Silicon in substrates can be in its high resistivity form or in porous state [88]. Typically, the wafers have a thickness between 300 [89] and 600 $\mu \mathrm{m}$ [90] but Huang et al. 2014 [91] succeeded in fabricating a thin-silicon film polarizer, whose overall thickness was less than $7.5 \mu \mathrm{m}$ with transmission losses less than $0.8 \mathrm{~dB}$.

Although scientists managed to reduce silicon thickness, its high refractive index of 3.42 still has a detrimental effect on the final polarizer operation because it is responsible for Fresnel reflections on the interfaces which affects Terahertz transmission and introduce unwanted reflections.

Quartz wafer [87], [92] (z-cut down to $500 \mu \mathrm{m}$-thick and refractive index 1.46) and glass (2 $\mathrm{mm}$ - thick and with refractive index 2.7) [93], [94] were also tested as promising substrate media, yet they exhibited shortcomings similar to those of silicon, owing to the relative high dielectric constant.

Dielectric polymers such as polystyrene and polymethylpentene appeared to be able to overcome these limitations, approaching the free-standing polarizers performance. Cetnar et al. 2012 [95] tested polycarbonate substrate (refractive index 1.56) resulting in good ER, although only until $300 \mathrm{GHz}$, above which IL rose. 
On the contrary, high density polyethylene (HDPE) is almost transparent and dispersionless at $\mathrm{THz}$ frequencies, with a refractive index of 1.526 [96]. Fast et al. 2011 [97] proposed Mylar, a polyester film with refractive index of 1.64, as an attractive way to get thin films (between 3 and $5 \mu \mathrm{m}$ thickness) and highly transmissive in the $\mathrm{THz}$ range. Among commercially available material, Shiraishi et al. 2015 [98] proposed Tsurupica resin with refractive index of 1.53, whereas Farid et al. [99] used Kapton film, a polyimide substrate (refractive index 1.5) that is nearly transparent at terahertz frequencies, although these materials are considerably more expensive. Finally, Partanen et al. 2012 [100] employed both cyclo - olefin copolymer ( $\mathrm{COC}$ ) with a refractive index similar to that of cyclo-olefin polymer (refractive index 1.53) and polymethylpentene (TPX) as base on which deposited the Al pattern. In parallel, there is at present a large interest in developing flexible components in electronics and communications, putting the focus on those substrates that also allow to obtain a flexible device. This trend has also reached higher frequencies and the field of wire grid polarizers. For example, in the THz range Ferraro et al. 2016 [101] obtained promising results with cycloolefin Zeonor polymer, with high mechanical flexibility, negligible birefringence and low absorption and reflection losses., Lai et al., 2020 [102] achieved ultrathin, highly flexible and optically transparent THz polarizer, by using an ITO (tin doped indium oxide) wire grid pattern and PET films (refractive index 1.65). Other attempts were made with more exotic materials, such as photo sheets [66] and common papers (refractive index of 1.47) [103].

Each of the proposed substrate category has both advantages and shortcomings that make them more suitable to specific experimental and performance requirements. Nevertheless, they can be roughly compared by considering their affordability (including, cost, availability, ease of use and fabrication) versus their performance (including thinness, internal reflections, $\mathrm{THz}$ transparency), as depicted in Fig. 9. 


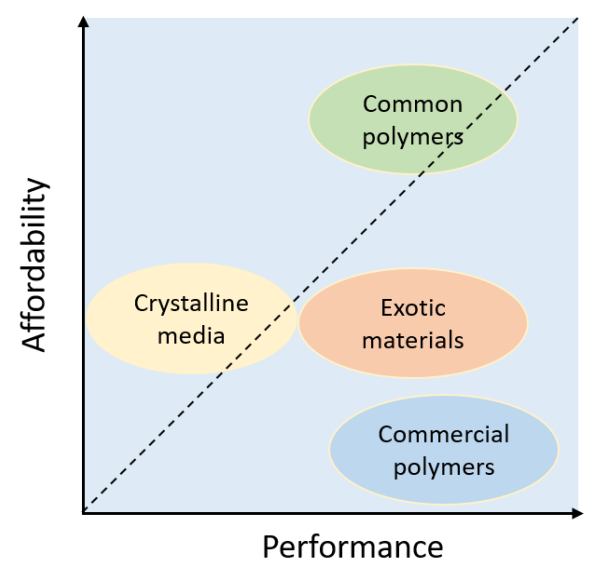

Fig. 9: Comparison among substrate tested materials for linear WGP. Common polymers seem to be more appealing than rare commercially available media, thanks to their high transparency to $\mathrm{THz}$ frequencies and the ease of achieving submillimetre thicknesses.

In the path to achieve low-cost components, the use of ink-jet printing to fabricate flexible polarizers exploiting the conductive properties of metal inks have been researched. These inks are usually based on Silver or Aluminium nanoparticles [67], [66]. The former type requires a rather complex and expensive process of fabrication in addition to specific printers of considerable cost [66], [104]. On the other hand, Aluminium based inks are more affordable, but they provide lower performance than Silver based ones [66], because of their higher resistivity $\left(\rho_{A g}=\right.$ $\left.1.59 \times 10^{-6} \Omega \mathrm{cm}<\rho_{A l}=2.65 \times 10^{-6} \Omega \mathrm{cm}[105]\right)$.

In substrate based WGPs the need of metals with high tensile strength is removed and so the range of metals that can be used to implement the wires is expanded. Thus, plenty of metals have been tested, considering their electrical conductivity, availability, deposition processes and cost. Deng et al. 2012 [106], Shiraishi et al. 2015 [98] and Lu et al. 2016 [107] went for gold deposition, thanks to its large absolute value of complex refractive index and long - term chemical stability. Isozaki et al. 2013 [108] mixed gold with chromium (Cr/Au: 5/75 nm). On the other side, copper was chosen by Tian et al. 2010 [109], Kishi et al. 2015 [110] and Yu et al. 2017 [111], since it had short skin depth that enabled deposition of extremely thin layers, being five times the skin depth of $93 \mathrm{~nm}$ at $0.5 \mathrm{THz}$. Despite the fact that both materials have the best electrical conductivity values, their high cost still represents a barrier in their widespread use. Silver was also considered as a good candidate for terahertz wire grid polarizers since it had excellent electrical conductivity and negligible absorption losses which compares it to gold. This versatile material has been used in shapes of metallized commercially available 
Nanopaste (composed of single - dispersion metallic NPs) [112], NPs printed with a Dimatix DMP - 2831 printer [99], films [89] and wires [88]. With lower cost, Aluminium (Al) has been proposed as an affordable metal for thin metal-film subwavelength-grating polarizers for application from UV/visible [94], [87], [113] [114] to the far IR and THz frequencies [97], [115], [100], [95], [101], [93] thanks to its low resistivity [90] compared with tungsten or chromium in this band, the availability of mature deposition techniques for its manipulation (it has a low melting point that is insensitive to evaporator conditions [92]) and it is cheaper than gold or silver. However, both Aluminium and Silver have been tested with coatings. Huang et al. 2013 [91] coated the aluminium grid with Benzocyclobutene, BCB, to prevent the metal further oxidation after the deposition process and to reduce Fresnel reflection with the air. On the other side, Shiraishi et al. 2016 [89] deposited $20 \mathrm{~mm}$ - thick layer of $a$-Si onto a $32 \mathrm{~mm}$ - thick Ag film. .

In addition to the selection of the materials, modifications to the basic design can be applied to enhance ER. The most popular solution adopted so far is the fabrication of double layer polarizer. The most used configurations are those gathered in Fig. 10.

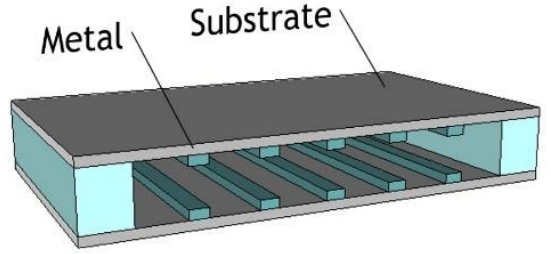

(a)

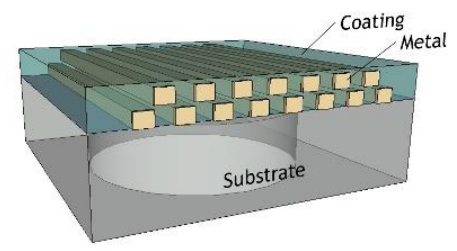

(c)

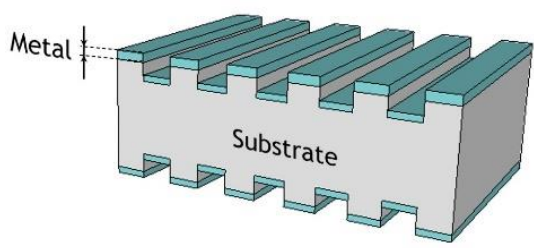

(b)

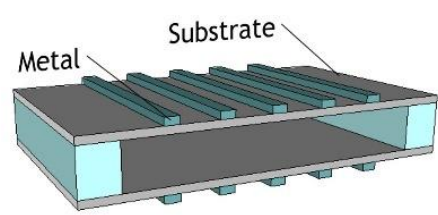

(d)

Fig. 10: From a) to d) most common polarizer arrangements to improve ER. Low losses and thin substrate as well as highly reflective metals are crucial to improve polarizers performance.

In the THz frequency, Yurchenko et al. 2008 [75] proposed an assembly in which two parallel gratings were attached to a frame, as displayed in Fig. $10 \mathrm{~d}$ ) bottom right. Isozaki et al. 2013 [108] took over the configuration and flipped the patterns putting them face to face (Fig. 10 a) top left). Another option was to pattern the wire grid on both substrate faces, as to imitate free standing structures [109], albeit there was an inevitable loss of ease of manipulation (Fig. 10 b) top right). 
Nevertheless, Lu et al 2016 achieved a 60 dB ER with a similar structure [107]. Huang et al. 2014 [91] attained the twofold advantage of increasing performance and reducing $\mathrm{Al}$ oxidation by inserting $\mathrm{BCB}$ as a $2 \mu \mathrm{m}$-thick spacer, obtaining strong coupling between the two grid layers, as shown in Fig. $10 \mathrm{c}$ ) bottom left. Shiraishi et al. 2016 [89] kept on with this idea and fabricated a double grating on one side of the substrate, separated by a layer of $a$-Si. Finally, Kishi et al. 2015 [110] proposed an alternative polarizer consisting of laminated metal-slit array on a cyclo - olefin polymer film, whereas Yu et al. 2017 [111] designed and fabricated a WGP sealed between two Si substrates. To further improve ER without affecting transmission and insertion loss inherent to the substrate, there have been several studies regarding observation of a kind of spoof Surface Plasmons Polaritons (SPPs), similar to those in the optical region, in subwavelength structures in the infrared and terahertz bands with some differences. As explained in Cetnar et al. 2012 [95], a pattern of periodical wires or apertures in a metal film can actually support these SPPs fulfilling specific geometrical conditions. This phenomenon was studied and applied to boost terahertz transmission of the permitted polarization state at certain frequencies [116], [117]. Tiang et al, 2010 [109] excited SPPs between 0.1 $3 \mathrm{THz}$, frequency range in which he achieved an ER of $22 \mathrm{~dB}$. by taking advantage of the coupling of dual micrometre pitch grating. On the other side, Middendorf et al. 2014 [92] achieved Extinction Ratio exceeding $60 \mathrm{~dB}$ by fabricating structures with an extremely high fill factor, approximately $90 \%$, (usually set to $50 \%$ ).

Technology-wise, the most popular fabrication techniques for substrate-based THz polarizers fabrication is photolithography followed by etching mode [90], deep ultraviolet interference lithography [118] or nanoparticles deposition [79], although they have the shortcoming of being relatively expensive and complicated processes.

All the efforts that have been put so far to develop optimized, robust reliable polarizers highlight the need of work that is still required to make these devices high-performance and affordable. The great variety of polarizing solutions aims at satisfying plenty of experimental conditions. In the following sections, scientific researches on this topic are presented. 


\subsection{Analysis of the $\mathrm{THz}$ response of a simple periodic graphite-based structure}

As reviewed in the previous section, several fabrication techniques and materials have been followed for the fabrication of $\mathrm{THz}$ polarizers. In this section an alternative low-cost approach is presented and the polarizing behaviour due to the dichroism effect in a periodic structure drawn using graphite-lead pencil on a common sheet of paper is discussed. These graphite-based periodic structures are characterized by standard time-domain terahertz spectroscopy (TDS-THz). Periodic grids of 1, 0.8- and 0.6-millimetre wire pitch are designed and studied. With the aim of enhancing the devised dichroism effect, different configurations are experimented, such as bi-layers grid, or double and triple stack grids.

\subsubsection{Graphite-based polarizer fabrication}

As previously mentioned, signals in the $\mathrm{THz}$ region correspond to wavelengths ranging from $30 \mu \mathrm{m}$ to $3 \mathrm{~mm}$, which could be defined as quasi-macroscopic. Pencil line widths are in the order of this wavelength range. Lead pencils are of interest due to the conducting properties of graphite crystal, that is estimated in 2-3 $\times 10^{5}$ $\mathrm{S} / \mathrm{m}$ for the c-plane and in $3.3 \times 10^{2} \mathrm{~S} / \mathrm{m}$ along the planes [119]. Even $\mathrm{THz}$ pulses were created by illuminating a pencil stripe on a paper with a femtosecond optical source [120]. Pencils with the highest content of graphite are more suitable for this application, so $8 \mathrm{~B}$ lead pencils will be used in the experiment.

Due to the transparency of the paper to the $\mathrm{THz}$ [65], [121] and the high graphite content of the pencil [122], the designed structures should behave as a conductive wire grid with a response in the $\mathrm{THz}$ frequency range.

To investigate this hypothesis, a graphite-based polarizer was fabricated, as shown in Fig. 11. The pattern with the required wire periodicity $d$ is drawn using an $8 \mathrm{~B}$ lead pencil on blank paper, that is used as a reference. 


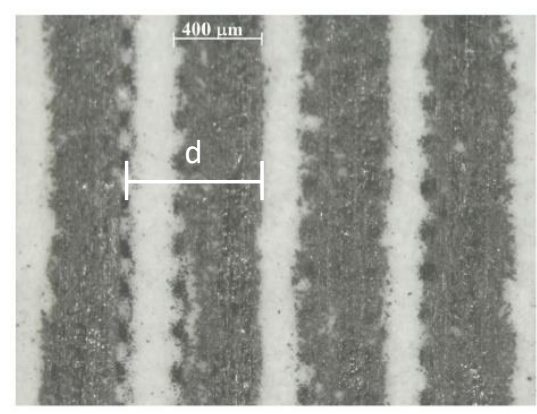

Fig. 11: Optical microscope image of the $0.8 \mathrm{~mm}$ pitch (d) grid structure with 0.4 millimetres wire width (fill factor ranging from 0.5 to 0.8 ). Black graphite wires alternate with blank paper spacings.

The samples are squares of $35 \mathrm{~mm} \times 35 \mathrm{~mm}$ with a fill factor ranging from 0.5 and 0.8 and a pitch of $1 \mathrm{~mm}, 0.8 \mathrm{~mm}$ and $0.6 \mathrm{~mm}$, corresponding respectively to an expected maximum frequency of polarization of $300 \mathrm{GHz}, 375 \mathrm{GHz}$ and $500 \mathrm{GHz}$. Polarizers of larger size can be easily developed.

\subsubsection{Experimental results}

To characterize the samples, a standard transmission fibre THz-TDS setup is used like the one in Fig. 11 is employed. Each grid is stuck on a graduated rotational holder placed in the free air path of the $\mathrm{THz}$ beam. The angle formed between the polarization axis of the incoming $\mathrm{THz}$ beam and the grid long axis, defined as the axis parallel to the wire direction, in relation to which the grid is symmetrical, is set by rotating the grid holder. For each sample, the $\mathrm{THz}$ time signal is recorded at 0 degrees (parallel condition), 45 degrees and 90 degrees (perpendicular condition).

The relative amplitude of the $\mathrm{THz}$ spectra (referred to the spectrum of a blank sheet of paper) is displayed in Fig. 12(a).

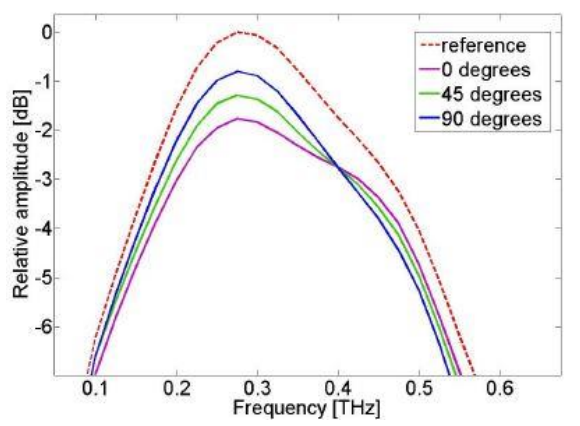

(a)

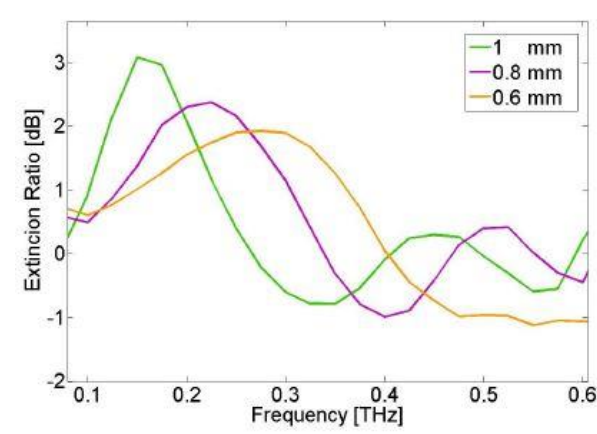

(b)

Fig. 12: (a) Frequency transmission spectra achieved by Fourier transforming the time dependent $\mathrm{THz}$ electric field. The dotted red line represents the reference blank paper signal, whereas the other three traces are the $90^{\circ}$ (blue line), $45^{\circ}$ (green line) and the $0^{\circ}$ (purple line). (b) shows the variation of the frequency of maximum ER as a function of the pitch dimensions.

As the incoming wavelength $\lambda$ is much greater than the grid periodicity $d$, a difference in the relative amplitude of the spectra is noticeable, ascribable to a 
polarization effect due to the selective absorption phenomenon (dichroism effect). The pitch determines the maximum frequency below which the effect is relevant. As it can be seen in Fig. 12, a decrease of the periodicity corresponds to a shift towards higher frequencies of the structure bandwidth. Fig. 12(b) shows the extinction ratio of the structures, here described as $E R=10 \log _{10} \frac{T_{\perp}}{T_{\|}}[100]$, where $\perp$ and $\|$ refer to the TM and TE polarization, respectively. With a single structure, an ER of only $3 \mathrm{~dB}$ is achieved (see Fig. 12(b)) which is far below the required features for a commercial polarizer, that is at least $20 \mathrm{~dB}$ [123].

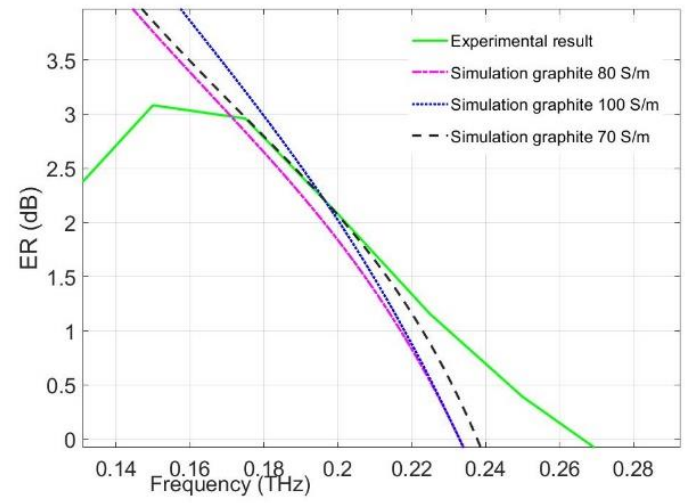

Fig. 13: Simulations of the graphite-based polarizer with $1 \mathrm{~mm}$ pitch, $0.4 \mathrm{~mm}$ wire width. Three different values were assigned to graphite conductivity as to better reproduce the low conductivity of the mix between clay, graphite and other materials.

Simulations were also performed as to assess the polarizing behaviour of the structure see Fig. 13. The agreement between simulations and experimental results is bounded to limited spectral region. This behaviour is probably related to the fact that the polarizer is tested in its limit operational region. The dynamic range of $\mathrm{THz}$ instruments based on photoconductive antennas falls abruptly at lower frequencies, as it can be seen in Fig. 13 below $0.18 \mathrm{THz}$. Thus, from one side, the instrument is unable to measure at low frequencies, and the polarizer, being at the limit of operation, reduces its performance as the frequency grows. Moreover, the deposition process could not ensure a uniform graphite deposition or composition, leading to an imprecise conductivity estimation.

The evaluation of transmission efficiency reveals a decreasing trend as the $\mathrm{THz}$ incoming wavelength becomes comparable with the grid periodicity. In addition, the transmission efficiency in the case of parallel condition exhibits a maximum of transmission for $\lambda=d$. Instead of the expected lowpass response, a bandpass behaviour is shown in Fig. 12, due to the bandwidth limitation of the $\mathrm{THz}$ characterization setup (low emitted power below $100 \mathrm{GHz}$ ). 
Finally, the degree of polarization is analysed (DOP). Fig. 14(b) shows that the graphite wire grid only partially polarizes the incoming $\mathrm{THz}$ beam, reaching a $40 \%$ polarization at the frequency of maximum ER.

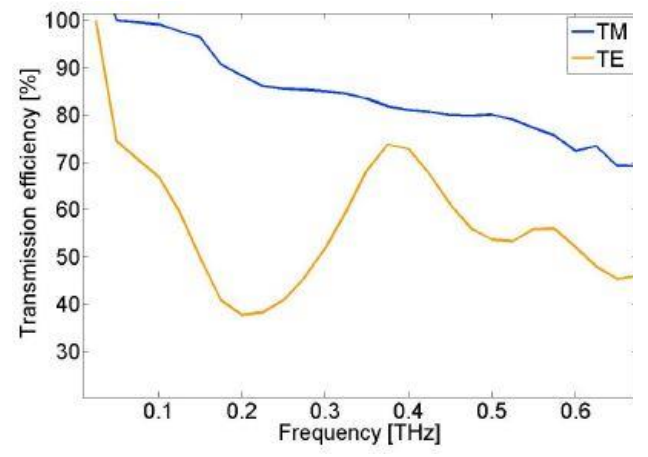

(a)

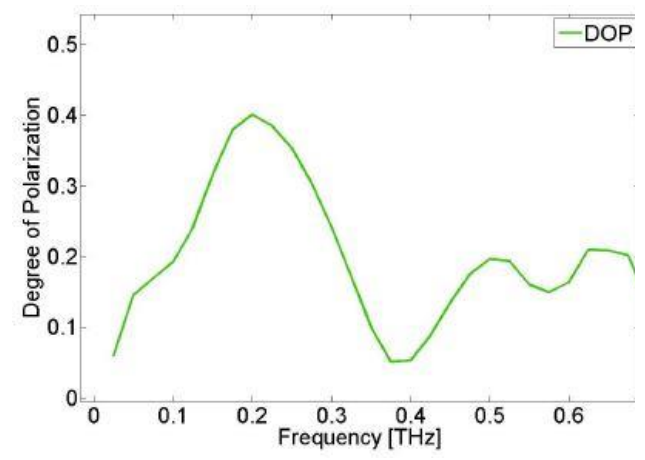

(b)

Fig. 14:(a) Transmission efficiencies for the $0.8 \mathrm{~mm}$ pitch structure. The maximum transmission efficiency exhibits a decreasing trend as the incoming $\mathrm{THz}$ wavelength becomes comparable to the pitch dimension. In addition to this behaviour, the minimum transmission efficiency shows a maximum of transmission when $\lambda=d$. (b) displays the Degree of Polarization of the $800 \mu \mathrm{m}$ pitch wire grid with a single layer structure.

The good conductivity of pure graphite is affected by the presence in the pencil of clay and other binders. The resistivity $\rho$ of the compound on the sheet of paper (measured as the averaged resistivity value of each drawn line) results to be 1694 $\Omega \mathrm{m}$. This value is several orders of magnitude above the pure graphite resistivity value that is $7.837 \times 10^{-6} \Omega \mathrm{m}$ [124] or common metals such as copper ( $\rho=$ $1.69 \times 10^{-8} \Omega m$ ). This last value of resistivity implies skin depths that range from $4.45 \mu \mathrm{m}$ at $100 \mathrm{GHz}$ to $2.57 \mu \mathrm{m}$ at $300 \mathrm{GHz}$.

Despite this poor conductivity, a polarizing behaviour can be observed. Thus, three different configurations have been tested for the $0.8 \mathrm{~mm}$ pitch pattern to improve its performance. The first design is obtained by depositing graphite on both sides of the same substrate (sheet of paper), whereas the other two structures are the result of sticking together two and three one-faced periodic structures, respectively. In these cases, particular attention is payed to superimpose them precisely. Results for the ER and the correspondent insertion loss, [125] are shown in Fig. 15. 


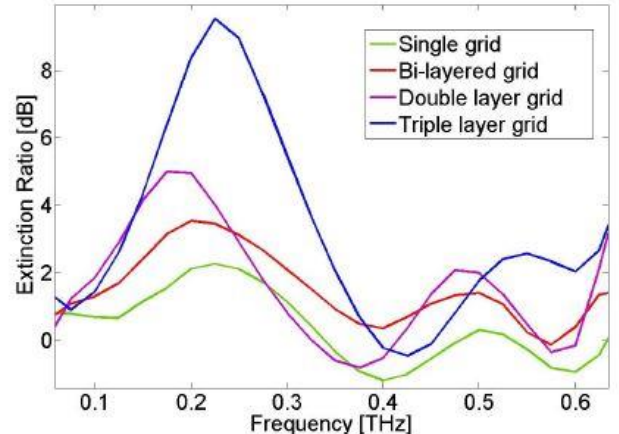

(a)

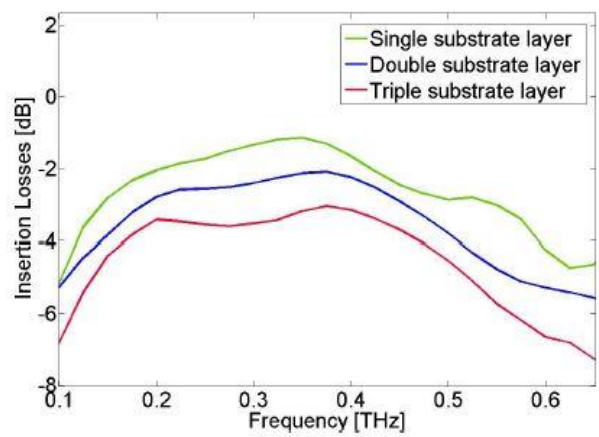

(b)

Fig. 15: The ER of three different structures and the corresponding IL are shown and compared with the basic one. The used wire grids are the $800 \mu \mathrm{m}$ pitch. The ER value increases as the number of structures grows, reaching almost $10 \mathrm{~dB}$.

The increase of the ER in the three-layer structure suggests that $\mathrm{THz}$ polarizers with moderate performance could easily be developed following this very simple approach. To test the durability of this method, the wire grid structures have been measured again after one month, checking that there have been no significant changes in performance.

\subsubsection{Conclusion}

In this section the potential of graphite-based polarizers in the $\mathrm{THz}$ has been investigated. The display of the selective absorption phenomenon when modifying the angle between the polarization axis of the incoming $\mathrm{THz}$ beam and the long axis, and the displacement of its maximum frequency according to the pitch variation hints at a polarizer behaviour. Extinction Ratio up to $9 \mathrm{~dB}$ are shown using an extremely simple low-tech approach based on low-cost materials with an estimated cost of less than one dollar.

This work was published in: M. Colleoni and B. Vidal, "Analysis of the $\mathrm{THz}$ response of a simple periodic graphite-based structure," Opt. Express, vol. 22, no. 24, pp. 30156 - 30160, 2014. 


\subsection{Flexible Sub-THz Metal Wire Grid Polarizer Based on an EGaln 24.5} Alloy

In this section, the potential for the fabrication of flexible low-cost $\mathrm{THz}$ polarizers based on conductive inks, in particular the case of eutectic alloy EGaln ${ }_{24.5}$ on a vinyl acetate substrate is described.

The EGaln 24.5 alloy shows low melting point, good wettability and high conductivity, resulting in a low-cost alternative to conductive inks based on metal nanoparticles. These properties are exploited to draw metal wire grid polarizers by using a technical pen on a bendable sheet of vinyl acetate monomer. This approach allows a quick low-tech and cost-effective implementation of flexible polarizers in the sub-THz band.

\subsubsection{EGaln 24.5 based polarizer fabrication: drawing}

The properties of the EGaln 24.5 alloy allows straightforward drawing of metal wire grids on flexible substrates using common technical pens, which behave as polarizers in the $\mathrm{THz}$ band.

The first step in the fabrication of the metal wire grid polarizer is the preparation of the EGaln 24.5 metal alloy. Following the procedure described by Zheng et al. [126], 99.9\% pure Gallium is melted in a beaker on a hot plate and then stirred with 99.9\% pure Indium with a glass rod. Metals are weighted 75.5:24.5 and they are kept in de-ionized water to reduce the development of oxide skin that specially characterizes Gallium and its alloys [127]. As the EGaln ${ }_{24.5}$ is liquid at room temperature and has high surface tension, sheets of vinyl acetate monomer offer good adherence, avoiding the formation of droplets of alloy that can blur the result [104]. Technical pens with highly precise nib widths can be used to deliver the ink by hand on the substrate. In particular, a technical pen with $0.8 \mathrm{~mm}$ nib size is used in the experiments [128]. The inner part of the refillable pen is previously treated with an $\mathrm{HCl}$ solution, trying to remove the oxide skin that quickly forms in the metal alloy as it comes in contact with oxygen. Additionally, in this way, the metal ink surface tension is reduced, making it to flow smoothly from the pen. The compatibility between the amount of alloy deposited with the pen and the high adhesion of the chosen substrate allows a direct drawing process. Several studies deal with the determination of the role of the alloy oxidation, when studying its rheological properties [104], [127], [129]. The solid-like response of EGaln ${ }_{24.5}$ and 
its high wettability are actually due to the compact, stable and homogeneous oxide skin that quickly forms on the droplet surface when it is exposed to air, avoiding the oxidation of the whole alloy (i.e. the alloy naturally passivates itself). The expected resistivity value of the eutectic alloy at room temperature is $\rho=$ $29 \mu \Omega \mathrm{cm}$ [126], [130], that is two orders of magnitude lower than that of silverbased inks in the same environmental conditions. The actual resistivity of the alloy used in the experiments is determined with the four-point probe method [131]. A $3.5 \times 3.5 \mathrm{~cm}^{2}$ thin layer of the alloy is deposited by hand on an acetate sheet. The wire thickness is estimated to be $(74.20 \pm 0.02) \mu \mathrm{m}$, but it is affected by the nib flux, whereas the substrate thickness is evaluated in $0.110 \mathrm{~mm}$ by mean of a digital calliper with an accuracy of $0.001 \mathrm{~mm}$ and a resolution of $0.02 \mathrm{~mm}$. Repeated measurements are taken in five different areas of the same sample to determine the homogeneity of the deposition process. The measurements provide a resistivity value of $\rho=(167 \pm 24) \mu \Omega \mathrm{cm}$. The deviation from the expected theoretical value is probably due to an excess of Gallium oxide in the alloy.

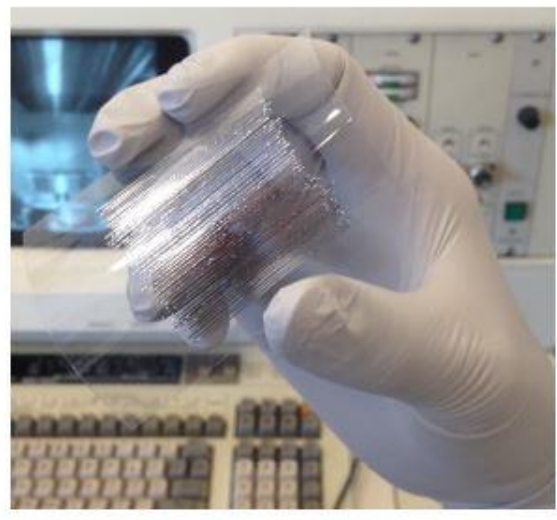

(a)

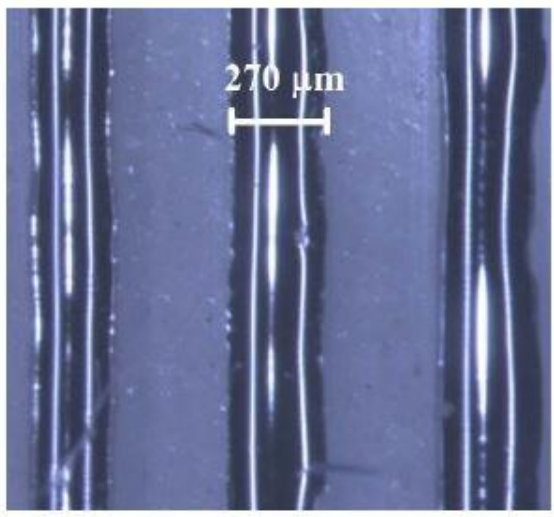

(b)

Fig. 16: Photograph of the bendable nominal $500 \mu \mathrm{m}$ pitch metal wire grid polarizer, with wire width ranging from $100 \mu \mathrm{m}$ to $300 \mu \mathrm{m}$ and a fill factor from 0.2 to 0.6 , based on EGaln 24.5 alloy on an acetate vinyl monomer sheet. b) Optical microscope image of the same structure.

Fig. 16 shows the periodic structure developed with the proposed technique.

Wires approximately $270 \mu \mathrm{m}$ wide and $3.5 \mathrm{~cm}$ long and with a nominal periodicity of $500 \mu \mathrm{m}$ is drawn on a vinyl acetate sheet. Wire and pitch standard deviation are estimated in approximately $23 \%$ and $24 \%$ of the nominal value. In order to achieve a polarizing behaviour based on the selective absorption phenomenon, i.e. the dichroism effect, a pitch lower than the incoming waves is required [79], [90]. 


\subsubsection{Experimental results}

To test the performance of the periodic structure as a polarizer the metal wire grid is stuck on a rotational holder and the transmission at normal incidence is analysed using a conventional all-fibre TDS-THz time domain spectroscopy setup, as the one pictured in Fig. 3. Measurements were performed with a single polarizer placed in the focus point of the optical system, consisted on four THz TPX lenses, plano-convex and bi-convex types. Fig. 17 compares the temporal waveforms of the substrate and a single polarizer in parallel and perpendicular orientations. The parallel configuration describes the one in which the angle formed by the long axis of the grid and the incoming $\mathrm{THz}$ electric field is 0 degrees, whereas in the perpendicular configuration this angle is 90 degrees.

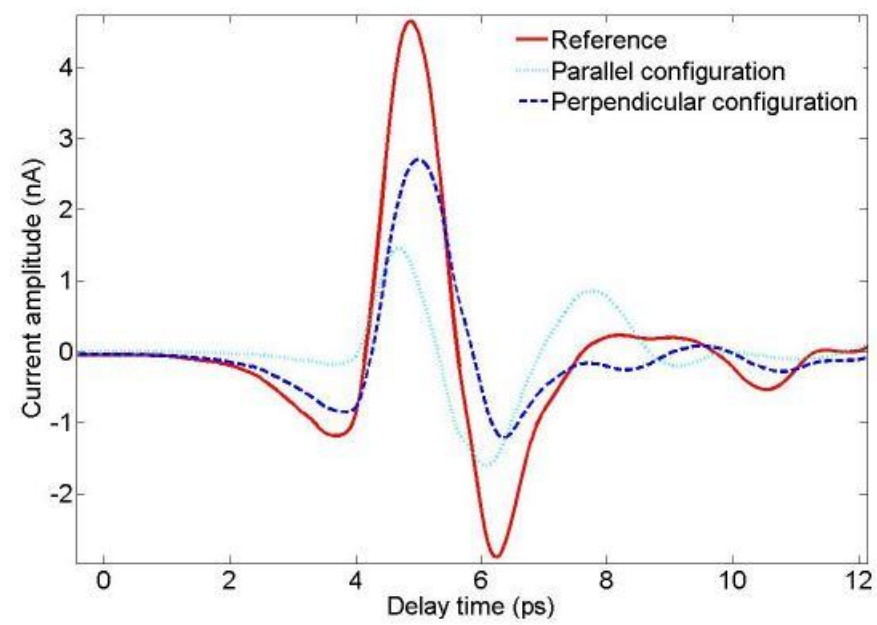

Fig. 17: Current signals comparison among the bare vinyl substrate and the signals of both perpendicular and parallel polarization.

The polarizer performance is expressed in terms of ER. Fig. 18 shows the transmittance of bare vinyl acetate, that is mostly transparent in the considered band. 


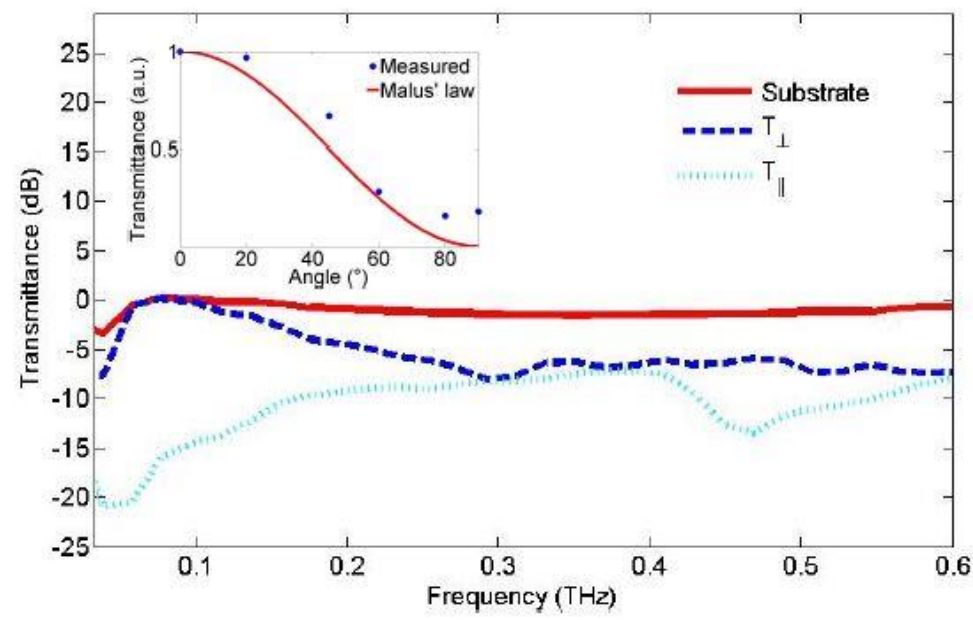

Fig. 18: Comparison of transmittance of both perpendicular and parallel configurations and the substrate of the $500 \mu \mathrm{m}$ wire pitch structure in the 0.1 - $0.6 \mathrm{THz}$ frequency range. Inset: Normalized transmittance as a function of the angle between the transmission axis of the polarizer and the incoming THz electric field at $100 \mathrm{GHz}$ and Malus' law.

A higher transmission coefficient is detected when the angle formed by the long axis of the grid (parallel to the wire direction) and the incoming $\mathrm{THz}$ electric field is 90 degrees, whereas a lower trend is achieved when the two directions are at 0 degrees. The inset of Fig. 18 shows the angular dependency of the transmittance, following Malus' law. Fig. 19 shows the corresponding ER trace in the frequency range between 0.1 and $0.6 \mathrm{THz}$.

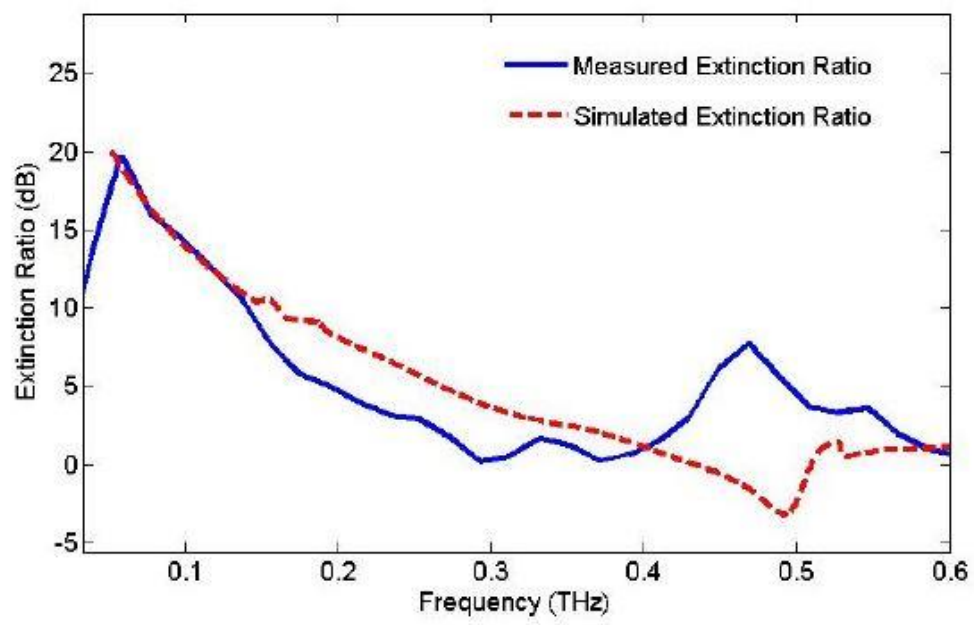

Fig. 19: Solid line: measured extinction ratio of the wire grid structure drawn with a target 500 $\mu \mathrm{m}$ pitch pattern in the $0.1-0.6 \mathrm{THz}$ frequency range. Dashed: simulations of a wire grid structure with irregularities both in the wire and pitch lengths.

The ER reaches up to $20 \mathrm{~dB}$, comparable with results collected in [123]. As expected, it reflects the transmittance behaviour in addition to a decay as the wire periodicity becomes of the order of the incoming $\mathrm{THz}$ wavelength. At low frequencies, the ER cannot be measured due to the very low power emitted by 
photoconductive antennas below $50 \mathrm{GHz}$. Simulations were performed with a FDTD simulation tool (CST Microwave Studio), for terahertz frequencies in Normal background condition. The measured optical parameters of the substrate and the alloy conductivity, as well as the irregularity of the wire width and pitch were also considered. To test the durability of the device, the polarizer went through a second set of measurements after a two-week period of exposition to air. No significant change in the ER evolution is seen. The ER shows just a small decrease of its maximum (less than $1 \mathrm{~dB}$ ) and the bandwidth is roughly the same. The capability to bend the polarizer has been studied following [132], where it was shown that bending a polarizer introduces Rayleigh-Wood anomalies that might degrade its performance. This anomaly is a redistribution of energy of forbidden diffraction modes of higher orders among the lower ones, so it negatively affects the transmission properties of the polarizer. It can be shown that above a given minimum bending radius the degradation can be avoided. This degradation due to strong bending of the structure has been tested by bending the polarizer. Equation (8) in [132]

$$
R>R_{\min }=\frac{S_{W G P}}{2 \arcsin \left|n_{s}-\lambda / \Lambda\right|}
$$

With SWGP (Single Wire Grid Polarizer) dimension and $\Lambda$ representing the pitch structure, states that the radius of the polarizer can be calculated in order not to incur in the anomaly in a specific frequency band. With a radius of curvature of 2.25 $\mathrm{cm}$, frequencies below $454 \mathrm{GHz}$ will show degradation due to this effect and a decrease of $7 \%$ in the ER peak amplitude was experimentally observed.

\subsubsection{Conclusion}

In this section, a low-tech and cost-effective method to easily develop polarizers in the $\mathrm{THz}$ band has been proposed. This approach is based on common, inexpensive and easily available materials. The combination of high electrical conductivity and good wettability makes EGaln 24.5 an efficient, low-cost and no toxic conductive ink that allows direct drawing of $\mathrm{THz}$ polarizers on flexible substrates. This approach could also be employed to draw other components such as $\mathrm{THz}$ mesh filters. Besides increased robustness in terms of normal and shear stress, the flexibility of both substrate and metal alloy offers the capability of implementing flexible electromagnetic components. Better performance in terms of repeatability (and therefore bandwidth) could be reached through the use of a 
more complex method for ink delivery based on hardware and software modifications on affordable commercial printers [133].

This work was published in M. P. M. Colleoni and B. Vidal, "Flexible Sub-THz Metal Wire Grid Polarizer Based on an EGaln 24.5 Alloy," in IEEE Transactions on Terahertz Science and Technology, vol. 6, no. 5, pp. 757-759, Sept. 2016. 


\subsection{Printing sub- $\mathrm{THz}$ wire grid polarizers using a composite liquid metal ink}

In the following, another fabrication method for EuGaln 24.5 based polarizers is described. A specific printer is indeed able to directly deposit the alloy on a suitable flexible substrate, reproducing the desired pattern with sub millimetre resolution.

\subsubsection{EGaln 24.5 based polarizer fabrication: printing}

A set of four $\mathrm{THz}$ polarizers was fabricated using a liquid metal printer [133] shown in Fig. 20.

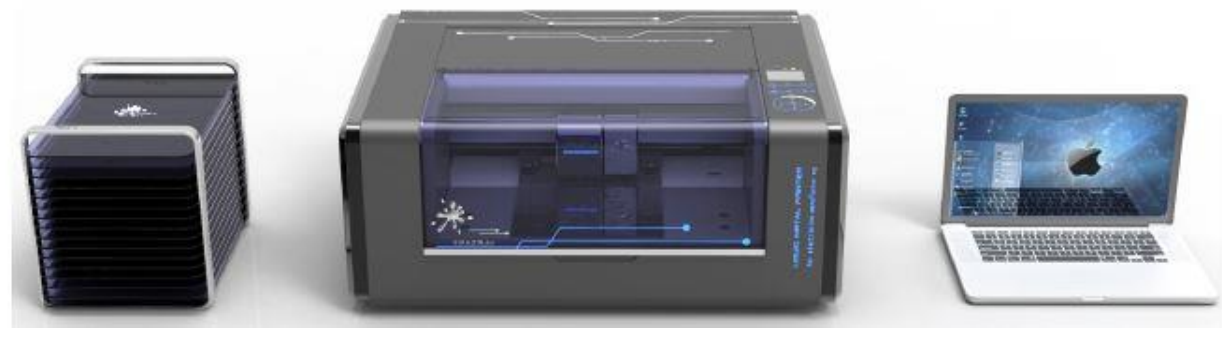

Fig. 20: Photo of the liquid metal printer based on the tapping-mode technique at room temperature.

Contrary to other expensive and power consuming techniques, such as the dispensing method for insoluble semiconducting materials or the laser sintering for inks based on metals with high melting point, the EGaln 24.5 ink can be delivered by liquid metal printer, based on a tapping-mode technique at room temperature. The alloy ink flows through a channel, in the printer cartridge, at specific pressure and speed till it reaches a roller ball, located just before the tip of the channel. The tapping motion of the channel, together with the rolling of the roller bead and the gravity effect, ensures a uniform ink outflow and a smooth deposition process. The key factors include an optical sensor that detects the distance between the roller bead and the printing substrate to adjust the tapping motion with a computer feedback mechanism, avoiding blurring and ink removals, and also to the relative planar simultaneous $\mathrm{X}-\mathrm{Y}$ displacement of the printing head and the substrate. This method allows the printing of extremely detailed patterns encoded in any format, with a resolution of $100 \mu \mathrm{m}$, at a printing speed of $0-400 \mathrm{~mm} / \mathrm{s}$ and printing pressure 0-800 g. Many materials such as PVC and PET are suitable for EGaln $\mathrm{n}_{24.5}$ ink deposition using this liquid metal printer. Their combination with the alloy ink creates a flexible structure able to resist to mechanical stresses, keeping its properties unchanged. 
The general scheme of all the polarizers is sketched in Fig. 21 .

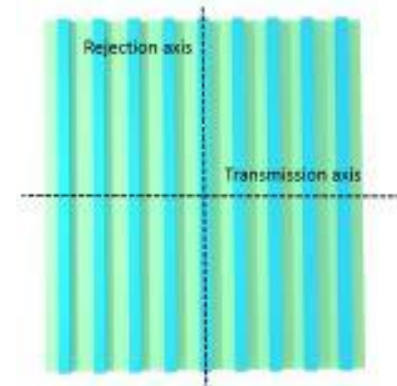

(a)

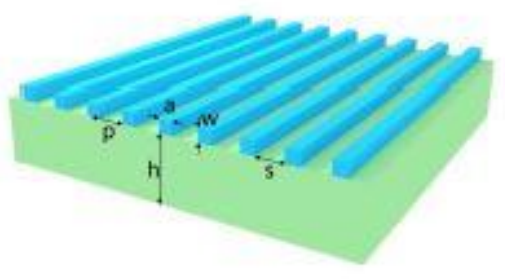

(b)

Fig. 21:a) Top view and b) perspective view of the array based structure: $p=p i t c h$, $a=$ wire width, $\mathrm{w}=$ wire thickness, $\mathrm{s}=$ spacing, $\mathrm{t}=$ substrate thickness.

An incoming $\mathrm{THz}$ beam with a component of the electric field parallel to the rejection axis induces a current in the wires due to the electrons that are allowed to freely displace along its entire length. As a consequence, only little energy is transmitted through the array, resulting in a reflected wave, rather than in a transmitted one. On the other hand, the $\mathrm{THz}$ wave is transmitted with little absorption when the $\mathrm{THz}$ electric field vector is parallel to the transmission axis.

Inspection with an optical microscope of the fabricated polarizers was performed to assess the fabrication procedure. A wire width and pitch of the first polarizer (P1) was estimated in (79.566 \pm 18.825$) \mu \mathrm{m}$ and $(300.624 \pm 27.494) \mu \mathrm{m}$ respectively, whereas the second polarizer (P2) the same parameters were $(93.652$ $\pm 15.497) \mu \mathrm{m}$ and $(207.586 \pm 18.551) \mu \mathrm{m}$ respectively. Fig. 22 stores images of polarizer P1.

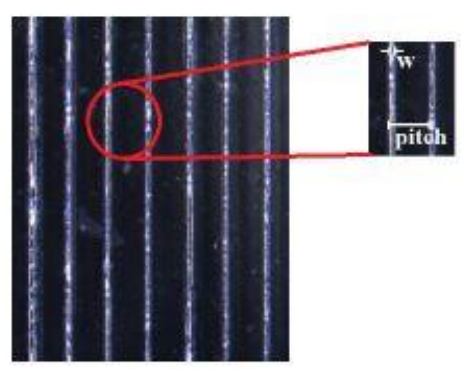

(a)

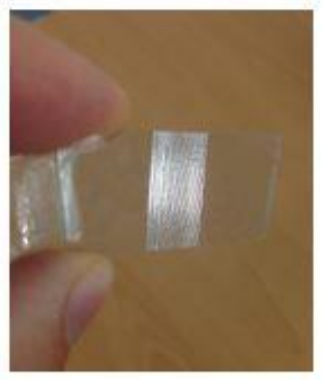

(b)

Fig. 22: a) Image of the bendable nominal $300 \mu \mathrm{m}$ pitch and wire width $w$ of $80 \mu \mathrm{m}$ metal wire grid polarizer obtained with an optical microscope and b) photograph of the full structure.

\subsubsection{Experimental results}

A home-made Time domain Terahertz set up (THz-TDS) was used to test the polarizing performance achieved by the fabricated structures. All the polarizers were tested with the following procedure. Each one was individually stuck on a 
rotational holder placed in the focal plane of described set up. The evaluation of the Extinction Ratio gave the performance level of the wire grid polarizers. The maximum transmission condition is achieved when the electric field of the impinging $\mathrm{THz}$ beam is perpendicular to the wire direction, i.e. it is parallel to the transmission axis of the polarizer. On the other hand, the minimum transmission condition is obtained when the $\mathrm{THz}$ incoming electric field is parallel to the wire direction, i.e. to the rejection axis (see Fig. 21 a).

Fig. 23 shows the comparison between experimental results and simulations driven with CST Microwave Studio software, for the two polarizers with nominal pitch of $300 \mu \mathrm{m}$ and $200 \mu \mathrm{m}$.

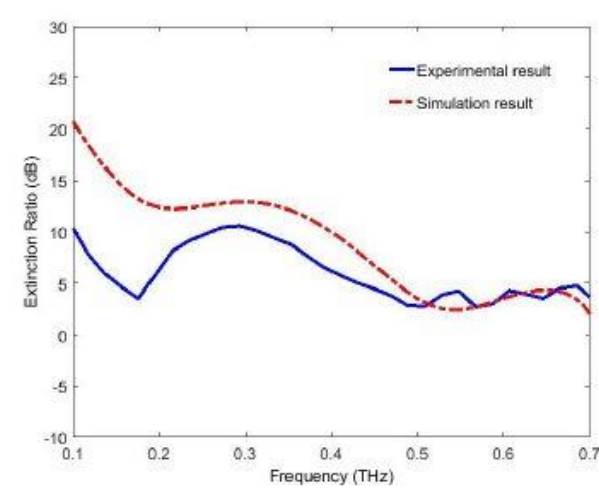

(a)

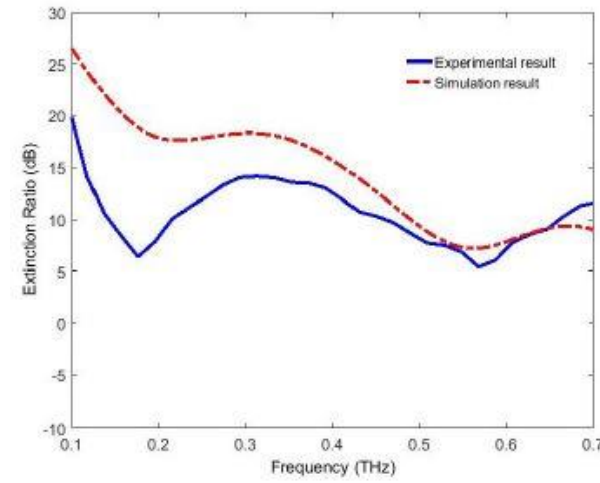

(b)

Fig. 23: Experimental results and simulations of the fabricated structures. a) $p=273 \mu \mathrm{m}, a=90$ $\mu \mathrm{m}, \mathrm{w}=30 \mu \mathrm{m} \sigma=3.4 \times 10^{6} \mathrm{~S} / \mathrm{m}$; b) $\mathrm{p}=225 \mu \mathrm{m}, \mathrm{a}=90 \mu \mathrm{m}, \mathrm{w}=30 \mu \mathrm{m}, \sigma=3.4 \times 10^{6} \mathrm{~S} / \mathrm{m}$.

The polarizing structures were simulated in the frequency domain in a normal background condition and with unit cell boundary conditions. The structure design was reproduced respecting the polarizers' geometrical parameters where possible. An estimated wire thickness of $30 \mu \mathrm{m}$ was simulated after considering that the skin depth of the alloy is estimated in approximately $0.737 \mu \mathrm{m}$ at $0.150 \mathrm{THz}$. Experimental results and simulations show slight discrepancies probably related to some irregularities in the pitch or in the deployed alloy quantity along the wire, that reduce especially the efficiency of the minimum transmission condition.

Further the performance of the polarizers of $200 \mu \mathrm{m}$ pitch was tested also by using them in an analyser configuration. One polarizer is put in the focal plane of the bi-convex lens, whereas the second one is put in the collimation path in front of the detector PCA. The transmission is measured as the angle between the transmission axis of the two polarizer is changed. Results are shown in Fig. 24 and they corroborate the idea that the structure only partially polarizes the $\mathrm{THz}$ incoming electric field, showing indeed almost no difference between the minimum 
transmission condition and the configuration in which the electric field vector forms a 45 degrees angle with the polarizer transmission axis. The check of the Malus' law confirms this shortcoming as the angles between the transmission axis of the polarizer approach zero.

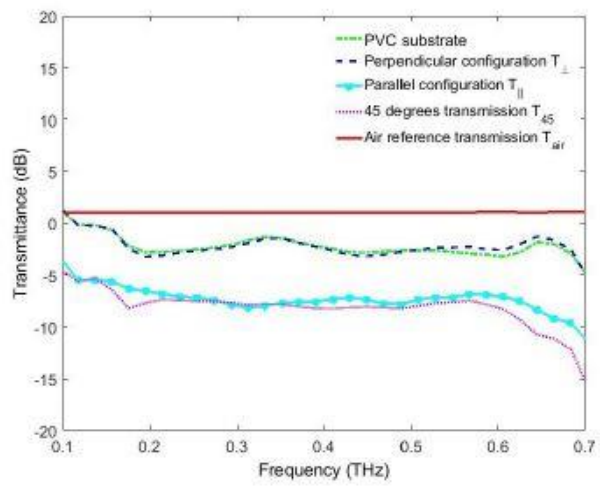

(a)

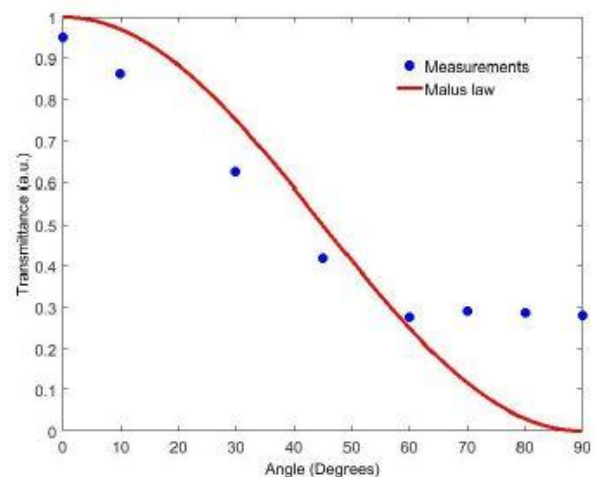

(b)

Fig. 24: a) Results of transmission in the analyser configuration of the second set of polarizers with pitch of about $200 \mu \mathrm{m}$, depending on their relative angle position. Comparison with air and PVC transmission. b) Malus' law check.

Finally, their flexible properties have been calculated, by means of equation (1), yet in this case periodicities of $0.2 \mathrm{~mm}$ and $0.1 \mathrm{~mm}$ have been considered. Thus, for the $0.2 \mathrm{~mm}$-pitch polarizer, degradation for frequencies higher than $0.75 \mathrm{THz}$ will be seen for radius of curvature higher than $56.6 \mathrm{~mm}$, whereas in the case of the $0.1 \mathrm{~mm}$-pitch polarizer the same happened at $1.5 \mathrm{THz}$ with a minimum radius of curvature of $153.3 \mathrm{~mm}$.

\subsubsection{Conclusion}

An innovative way to achieve a sub-THz wire grid polarizer has been presented. An ink-jet printer modified to selective deploy the electronic eutectic Galn ${ }_{24.5}$ on PVC substrate was employed for directly writing sub-THz polarizers. Two sets of polarizers with different pitches were printed and its transmission ratio was measured in two key positions to calculate the corresponding ER. Results show that an ER up to $14 \mathrm{~dB}$ is achieved, ensuring a good polarizer behaviour. Furthermore, the set of polarizers with shorter pitch is also tested in the analyser conditions, revealing some shortcomings of the printed structure, probably due to the irregular amount of alloy along the deposited wire or in the pitch reproducibility. So that further studies are required in order to improve polarizing performance at smaller angles in the analyser configuration, where the angles formed between the transmission axis of the two employed polarizers approaches zero. Additionally, the 
fabrication method can be extended to print other passive components such as filters.

This work was published in: Margherita M. P. Colleoni, Qian Wang, Jing Liu, and Borja Vidal "Printing sub-THz wire grid polarizers using a composite liquid metal ink", Proc. SPIE 10103, Terahertz, RF, Millimetre, and Submillimetre-Wave Technology and Applications X, 101031T (24 February 2017). 
3 Fast quantitative characterization of defects with fixed-delay $\mathrm{THz}$ sensors

As described in Section 1, there is a strong interest in widening the range of applications of $\mathrm{THz}$ waves and one of the areas that is getting more and more attention is related to non-destructive testing and monitoring in industrial applications.

This section is devoted to the application of Terahertz system in the industrial quality control operation, one of the most promising application of this technology so far.

\subsection{Fixed delay sensing}

Inspection time can be considerably reduced if Optical Delay Line (ODL) scanning is avoided in applications where single point measurement provides enough information [3]. Thus, instead of detecting defects from the time-domain THz trace, the ODL can be fixed at a given time position in such a way that optical pulses at the receiver antenna sample always the same point of the THz pulse. Therefore, small changes in the time-of-flight of the pulses can be measured from variations in the current signal in almost real-time. By monitoring deviations from the known signal, it is possible to detect defects and therefore determine which items should be discarded.

The concept of fixed-delay techniques to enhance the speed of $\mathrm{THz}$ measurements was proposed in 1D in [21] to monitor changes in additive content in the compounding of polymers. Recently, the option of excluding the scanning 
delay line, with the aim of further speed up the packaging scanning have been proposed to keep on paving the way to the $\mathrm{THz}$ industrial applications [134].

In this section, the extraction of information from defects in samples made of non-polar non-dispersive dielectrics (such as plastic, ceramic, glass, composites) using a fast fixed-delay configuration in a $\mathrm{THz}$ photoconductive platform is investigated. 


\subsection{Principle of operation}

In pieces made of materials with relatively constant optical parameters, defects such as dents, voids, protrusions and contaminations within the $\mathrm{THz}$ beam result in a change of the effective refractive index and consequently an Optical Path Difference (OPD), i.e. a change in the Optical Path Length (OPL) given by $\Delta n \Delta d$.

This variation in OPD translates into a change in the time delay $\Delta t$ experienced by the THz pulse, as shown in Fig. 25 . It is expressed by:

$$
\Delta t=\frac{\Delta n \Delta d}{\mathrm{c}}
$$

where $\Delta n$ is the difference in refraction index between the core material and the defect, $\Delta d$ is the depth of the defect and $c$ is the speed of light in vacuum.

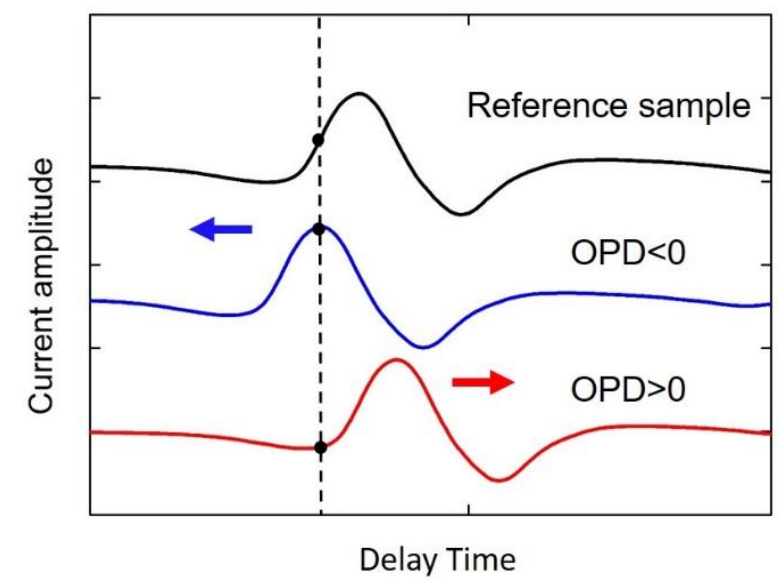

Fig. 25: The OPD experienced by the $\mathrm{THz}$ pulse varies depending on whether there is a mechanical defect inside a dielectric piece. The pulse is accelerated or retarded, according to the nature of the defect, i.e. on $\Delta \mathrm{n}$. The dashed line indicates the fixed time position.

As mentioned in Section 3.1, instead of conventional time-of-flight measurements from the $\mathrm{THz}$ pulse trace, the ODL can be kept at a fixed time delay to boost acquisition time. Thus, defect information is extracted from the timedependent current signal, as shown in Fig. 26. If the reference sample is known, this approach allows not only the identification of defects but also their characterization and quantification. 


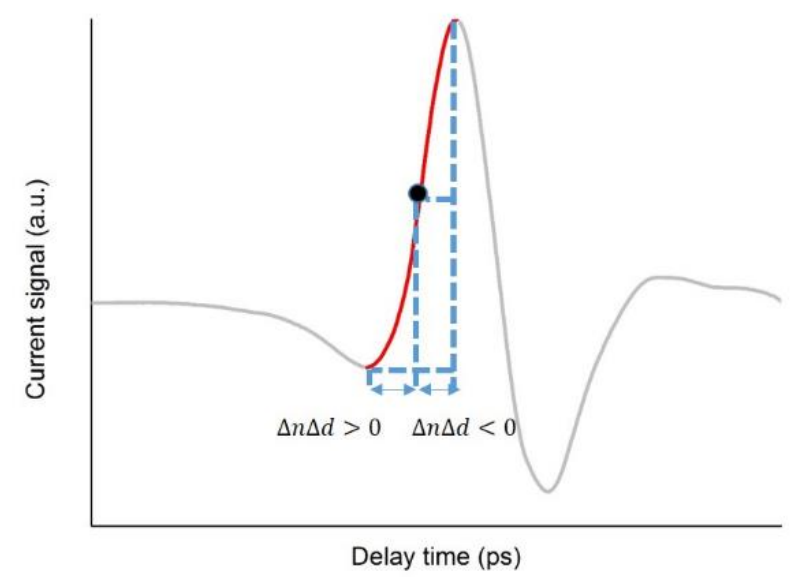

Fig. 26: Fixed-point rapid THz measurement concept and range of measurement.

To relate current to time changes, a complete trace of the $\mathrm{THz}$ pulse is firstly obtained in a homogeneous portion of a reference sample. Under the assumption that the only change experienced by the pulse will be in terms of time shift, the reference signal will allow to associate an OPD to each measured current value. To accomplish this assumption, the reference pulse data is fitted by a fourth-degree polynomial in the opposite axis of the original measurement (Fig. 27).

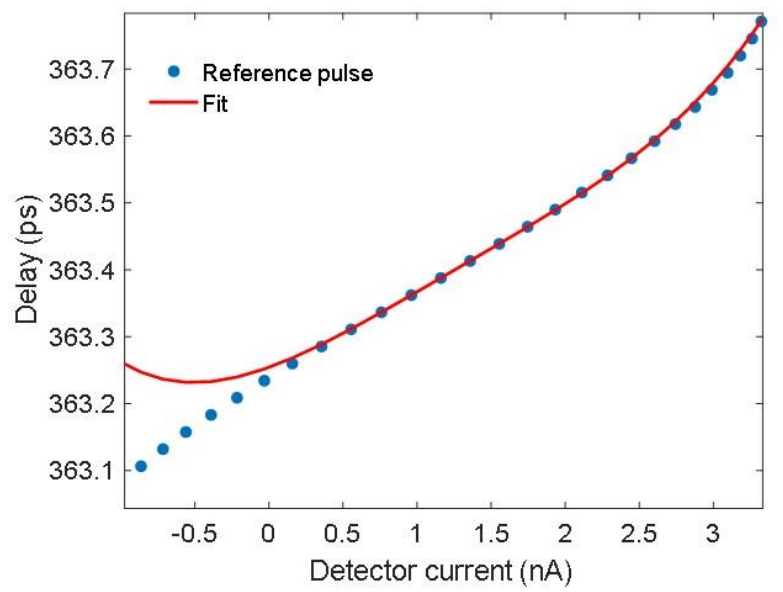

Fig. 27: Polynomial fit to the range of current values involved in the measurement. Axis are exchanged respect to the original measurement allowing for direct conversion from current to delay.

In addition to the detection of the presence of defects, the method can also be used to extract information from the distinctive current profile induced by the item. The sign (direction) of the time shift experienced by the $\mathrm{THz}$ pulse provides information about the nature of the defect. Pulses shifting towards left indicate samples with scratches or internal voids, whereas a bump on the surface of the sample will shift the pulse towards right. Contamination with materials with different refraction index can also be detected in this way. 
Furthermore, the volume of the defect can be estimated. OPD changes are related to the variations in refractive index occurring in the volume defined by the $\mathrm{THz}$ beam spot area and the defect thickness. The relation between the change in time delay caused by the defect and its volume can be estimated using an effective medium approximation (EMA). The refractive index of the medium $n_{\text {eff }}$ experienced by the $\mathrm{THz}$ beam could be described by Wiener bounds, where maximum screening of a microstructure is considered [7]. In this case, the effective refractive index would be $n_{e f f}=f_{r e f} n_{r e f}+f_{d} n_{d}$ where $f_{r e f}$ and $f_{d}$ are the volume fractions of the reference and defect material inside the considered beam volume and $n_{r e f}$ and $n_{d}$ are their corresponding refractive indexes. Then, assuming that usually the composition of the object sample is well-known and that the main degradation is due to internal voids filled with air, it is possible to set $n_{d}=n_{\text {air }}=$ 1 and $f_{d}=f_{\text {air }}$. Since the volume fraction must add up to one, the effective index can be expressed as:

$$
n_{e f f}=n_{\text {ref }}+\left(1-n_{\text {ref }}\right) f_{\text {air }} .
$$

The volume fraction of the void $f_{\text {air }}$ is the ratio between its volume and the total problem volume. For a circular gaussian beam, this volume can be approximated by a cylinder with a radius $r_{\text {spot }}$ and a depth $\mathrm{d}$ as the defect thickness $f_{\text {air }}=$ $V_{\text {air }} / \mathrm{d} \pi r_{\text {spot }}^{2}$.

By combining expressions (2), (3) and the above definition of the defect volume fraction $f_{\text {air }}$, the volume of the void inside the spot can be described as:

$$
V_{\text {air }}=\frac{\Delta t c \pi r_{s p o t}^{2}}{n_{r e f}-1}
$$

The variation of the thickness of the sample $\Delta d$ along the scanning path can be obtained with this technique as well, so that a touchless profilometer can be implemented. In fact, the thickness variation $\Delta d$ can be related to the time delay variation $\Delta t$ by substituting $\Delta n=n_{\text {ref }}-n_{\text {air }}=n_{\text {ref }}-1$ in (2), obtaining:

$$
\Delta d=\frac{\Delta t c}{n_{r e f}-1}
$$

\subsubsection{Performance analysis}

The resolution of the technique depends on the minimum change that can be detected in the current signal, which in turn is limited by noise as well as by jitter from the laser and the ODL. To assess the minimum current change that can be 
detected in the experimental setup, long-term measurements of current amplitude at a fixed time delay were performed (see Fig. 28). After stabilization, a maximum change of 41 pA over a seven-hour time period is recorded. As it can be seen in Fig. 28 , it is quite sensitive to temperature, so the optical source needs to be in a place with controlled temperature. Fortunately, thanks to optical fibre, it can be located far away from the sensor head [135].

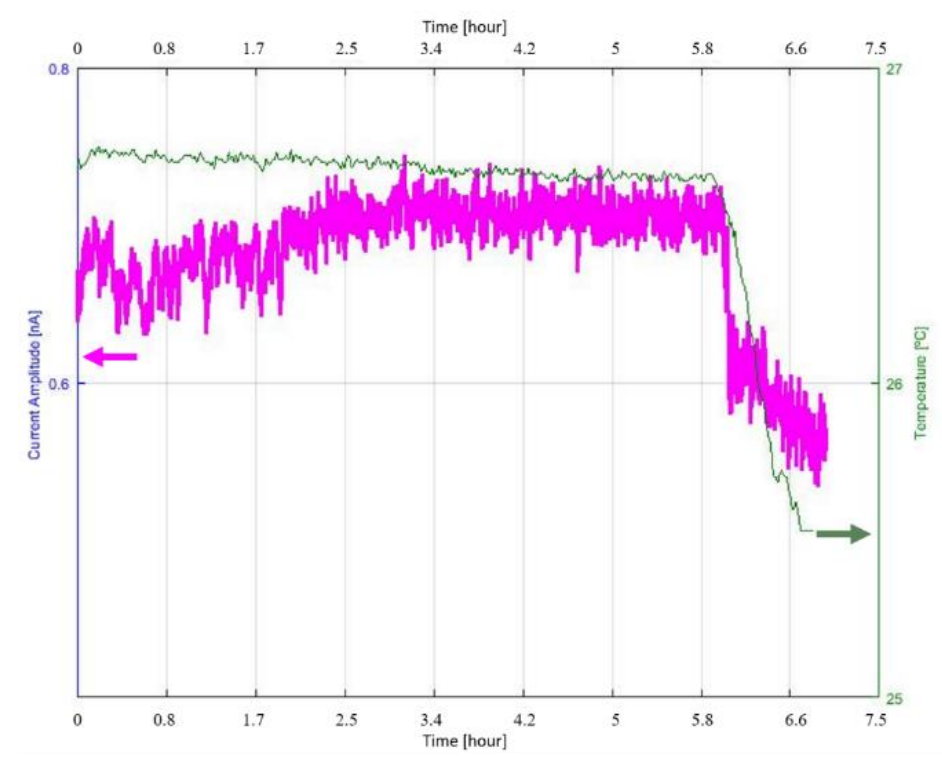

Fig. 28: 7 hours long-term measurements of current amplitude at a fixed time delay. Pink line refers to current amplitude fluctuations (left axis), whereas green line indicates the temperature variations (right axis). The abrupt fluctuation could be related to the temperature variation.

This stability translates into a delay change that depends on the slope of the temporal pulse. The current setup produces pulses with a slope of $0.13 \mathrm{ps} / \mathrm{nA}$ (Fig. 27) and a collimated beam spot with radius at $1 / e^{2}$ of $7.25 \mathrm{~mm}$ which gives a minimum volume detection of $0.44 \mathrm{~mm}^{3}$.

The theoretical maximum detectable volume is bounded by the maximum delay measurable at the edge of the pulse, as shown in red in Fig. 26. Beyond this range, if the type of potential defect is known, e.g. due to knowledge of the fabrication process all defects might be voids, it is possible to notice that there is no sample or if it is defective but not to extract information about the defect. Another limit on the maximum measurable defect volume, is given by the validity of the effective medium approximation.

Effective medium theory is used to describe heterogeneous media whose components are mixed at subwavelength scale. In the present approach, the underlying physical phenomena is the interference between two pulses very slightly delayed one respect to the other. One of them crosses the uniform 
reference material and the other one goes through the defect; therefore, each pulse experiences a different delay. If the defect is comparable in size to the beam spot, this interference would deform the pulse and make the fixed-time-position technique invalid. The assumption regarding changes in the $\mathrm{THz}$ trace being caused only by time delays is valid for a range of defect sizes. Since the beam over the defect is being attenuated by scattering, the slope of this THz pulse is smaller than in the homogeneous reference. Associating a time delay to the measured current using the reference pulse will underestimate the delay change produced so that $\Delta t_{\text {real }}=\Delta t_{\text {ref }} / \alpha$, where $\alpha$ is the relative attenuation between reference pulse and pulse over the defect. The effect of this fact on the approximation was estimated by measuring full traces over the largest defect in the set considered for this paper and a value of $\alpha=0.9$ was obtained. This means that, at most, an error of $10 \%$ is being committed due to scattering effects.

Using this fixed-delay approach, acquisition speed can be fast. For example, using a conventional lock-in amplifier for data acquisition, with a time constant of $30 \mathrm{~ms}$, and considering a typical speed of a conveyor belt, $3.2 \mathrm{~m} / \mathrm{min}$ [136], one measurement can be recorded at $1.6 \mathrm{~mm}$ spatial intervals. Being based on measuring OPD, this method is insensitive to changes in the position of the sample along the $\mathrm{THz}$ path.

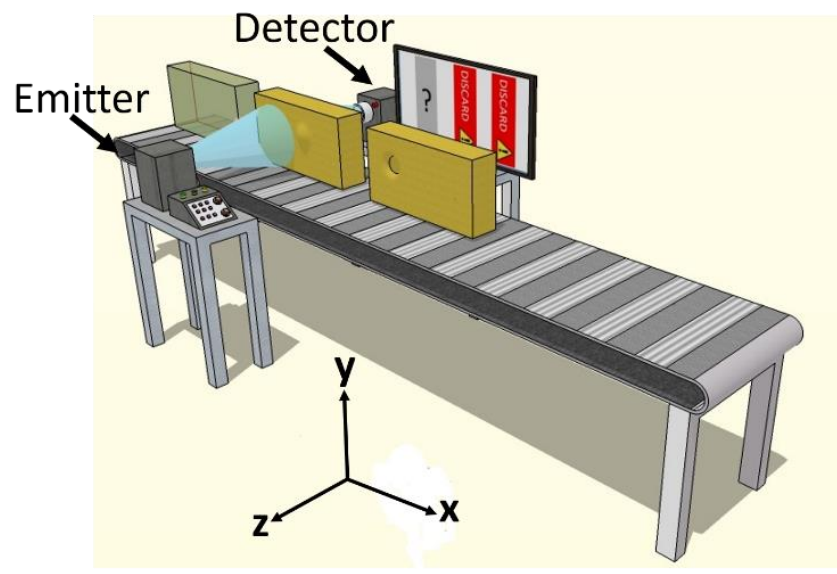

Fig. 29: Potential application of the concept of the single-element fixed-delay method in an industrial environment.

\subsection{Experimental results}

Tests have been done to validate the feasibility of the technique using the setup shown in Fig. 30. 


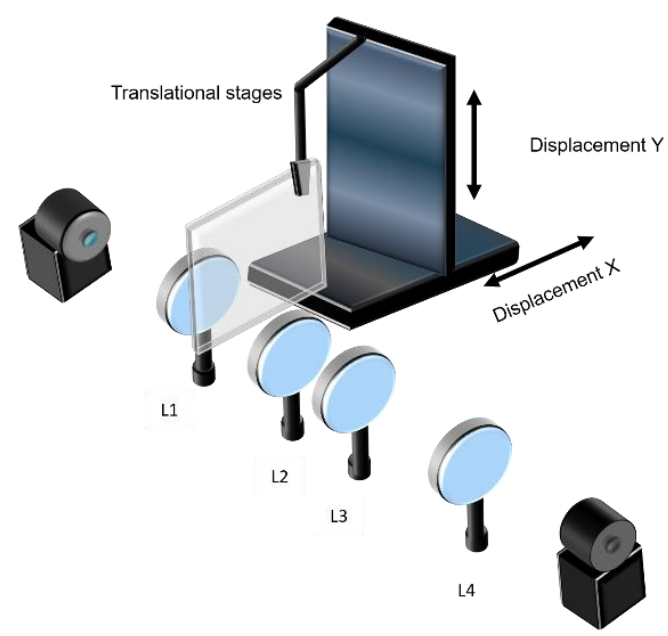

Fig. 30: Sketch of the measurement setup and the movement of the hypothetical defective piece through the optical system.

As the rest of $\mathrm{THz}$ characterizations in the Thesis, it is based on an all-fibre Time Domain Spectroscopy (TDS-THz) setup based on photoconductive antennas in transmission configuration. The basic setup was modified to include two translational stages mounted perpendicular to the $\mathrm{THz}$ optical path to reproduce the transverse displacement of a sample. The sample falls on the first collimated area of the optical system defined by L1 and L2, as depicted in Fig. 30.

\subsubsection{Void detection and characterization}

A first test of the capability of the method to identify the presence of a void was carried out. A translucent polypropylene (PP, refractive index 1.498 [1]) piece has been drilled to create a small channel of $(1.29 \pm 0,09) \mathrm{mm}$ in its interior (see inset in Fig. 31). Then, the sample crossed the THz spot (transverse direction). Fig. 31 shows the current profiles of both samples. The current profile of the reference PP sample kept a uniform height along the scanning direction, corresponding to the reference sample. On the other hand, the current amplitude of the defective piece showed an irregular trend with a drastic drop compared with the homogeneous sample at about $6 \mathrm{~mm}$ from the left edge. 


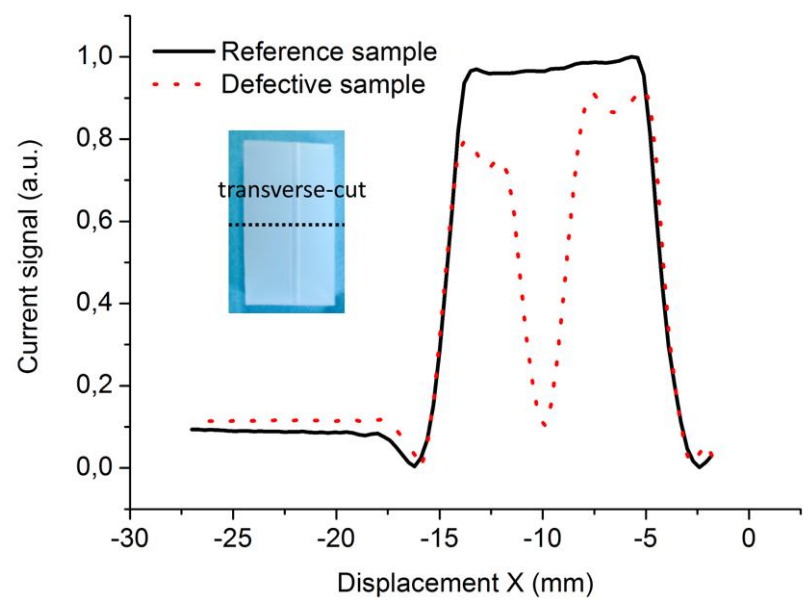

Fig. 31: Current profile along the dimension in the $x$ direction for the reference sample (black solid line), for the defective piece (red dashed line). Inset: defective piece with an inner drilled channel filled with air.

Further experiments have been carried out on samples of methacrylate, with a refractive index of 1.65 , with voids of different sizes. The volume of these defects has also been estimated using Optical Coherence Tomography (OCT).

As an example, the delay derived from the measured current trace is shown in Fig. 32 for a void of specific size.

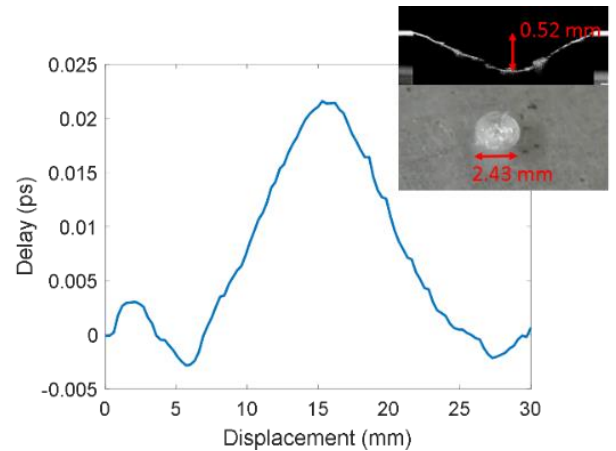

(a)

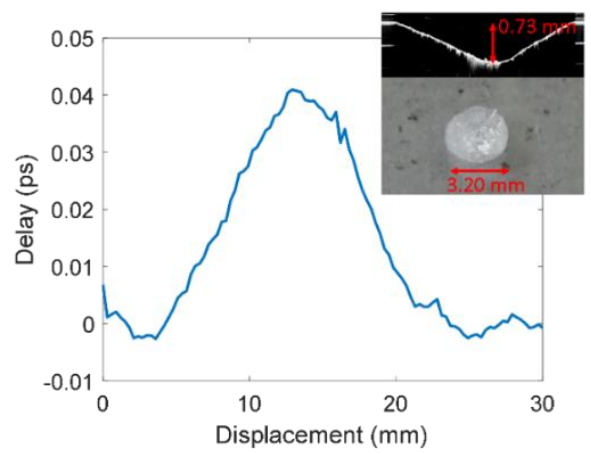

(b)

Fig. 32: Time delay measured when scanning over two defects of different volumes. a): 0.90 $\mathrm{mm}^{3}, \mathrm{~b}$ ): $2.10 \mathrm{~mm}^{3}$. Insets: Tomographic (top) and optical (bottom) images of the defects with an estimation of its dimensions.

To estimate its volume, the delay measured at the maximum of the delay profile from scans of seven defects has been correlated to their real volume. In Fig. 33, a good linear correlation can be observed for all but the last defect. This is mostly due to the reasons explained in Section II and mark the upper volume limit of the setup. Similarly, below $0.5 \mathrm{~mm}^{3}$, no measurement was possible. For the first six points, a linear fit can be performed, and its slope can be related, using (4), to an estimate 
of the beam radius. A value of $(5.7 \pm 0.5) \mathrm{mm}$ is obtained, close to the measured $1 / \mathrm{e}^{2}$ radius.

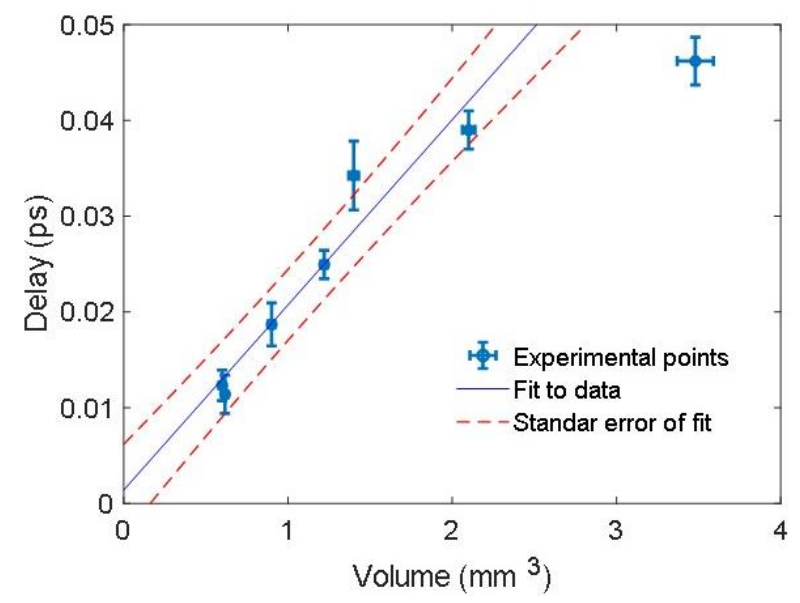

Fig. 33: Delay variation measured at the top of the current profile for each void versus its actual volume measured with OCT imaging. Fit excludes the point with the largest volume.

In Fig. 34 the overall process is shown for the sake of completeness.

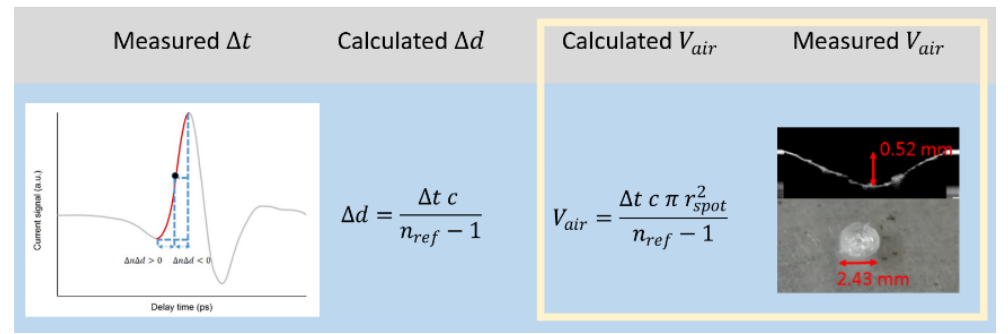

Fig. 34: Summary of the overall process followed to estimate the defect volume. The estimation of the volume of the defect is compared with its direct measurement (parameters inside the square).

\subsubsection{Thickness measurement}

The next measurements were intended to test the capability of the setup to sense thickness changes along the sample. To accomplish this, a second methacrylate sheet $5.18 \mathrm{~mm}$ thick with homogeneous surface was placed in the way of the $\mathrm{THz}$ beam and scans like in the previous experiment were performed. This time, however, to prevent the beam from going over the edges of the sheet, the measurement was carried out in the focused zone of the setup where the beam radius is smaller at $1.5 \mathrm{~mm}$. After that, the thickness of every point relative to the thinnest one was computed using (5). Then, a calliper with micron resolution was used to measure the sample thickness along the same path followed in the $\mathrm{THz}$ scan. Fig. 35 shows the comparison of the two methods showing good agreement between them. 


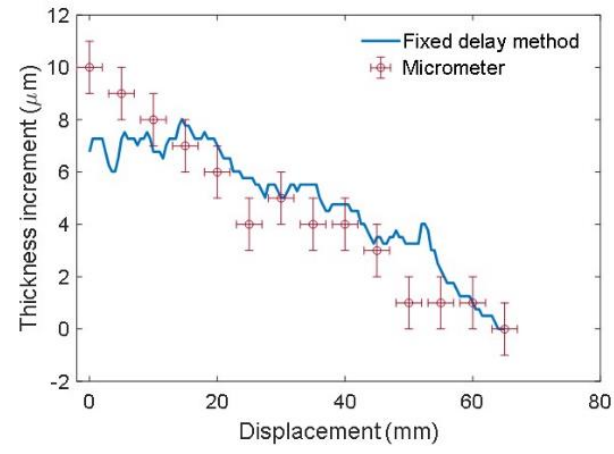

(a)

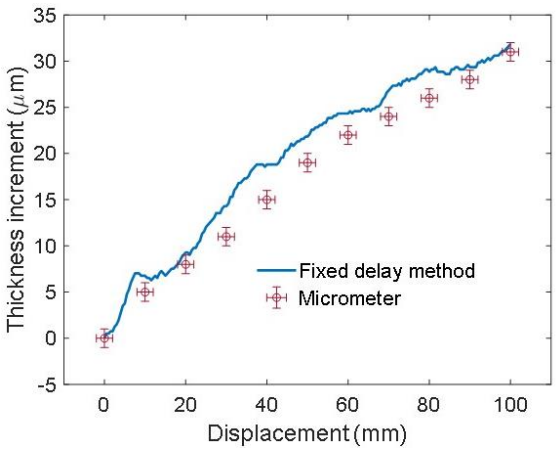

(b)

Fig. 35: Thickness measurement performed along two paths in a transparent methacrylate sheet.

\subsection{Conclusion}

The potential of a fixed-delay configuration for the characterization of defects through $\mathrm{THz}$ waves has been investigated. It has been shown that, in some ranges, it can be used to estimate the void volume as well as changes in thickness of the sample. Avoiding the need to record the entire $\mathrm{THz}$ trace, the measurement time is reduced, and valuable information are extracted. This approach can be useful for inline quality control equipment in some industries to replace more complex tools such as those based on radioactive sources. Further work is needed to test the method in relevant environments. 
Dielectric-based Components and Methods for Terahertz Sensing 


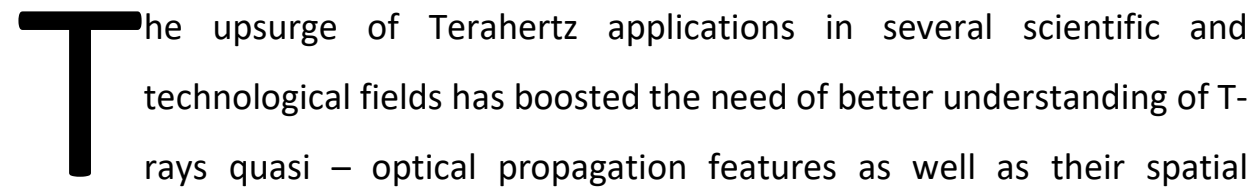
distribution. It is well-known that misalignment in the optics elements and a poor beam shape could lead to bad results, with narrowed spectral bandwidth detection.

There are no ad-hoc techniques for $\mathrm{THz}$ beam profiling, thus optical methods have been borrowed and partially adapted. However, some issues must be considered, especially when investigating sub - wavelength spatial resolution, when the system exhibits strong diffraction.

\subsection{Beam profiling fundamentals}

Beam profiling has become a routine procedure in a wide range of optical systems. In 1971, Arnaud et al. [137] described the first Knife Edge (KE) method to quickly and accurate measure the beam waist of a gaussian beam, and since then several techniques have been proposed. Other systems include optical imaging of the focused spot and a computational determination based upon interferometric measurement of the far field pattern [138], ray tracing, multiphoton ionization and fluorescence correlation spectroscopy [139], but these gradually fell out of favour because they were either too complicated or constrained by specific experimental procedures. Therefore, more affordable methods were preferred and nowadays, beam characterization is mainly performed using camera-based systems or 
aperture scanning methods. Next, a brief review of the most common beam characterization methods is provided for $\mathrm{THz}$ beams.

Camera-based systems, being routinely used in optical wavelengths, are still an issue for beam profiling in the $\mathrm{THz}$ range due to the larger wavelength of $\mathrm{THz}$ waves. They enable real time results and avoid mechanical parts [140], albeit their use implies in most cases a rearrangement of the existing set up [141]. So, in the $\mathrm{THz}$ range the use of cameras of beam profiling is rather limited, except for specific commercial products [142], [143], [144]. Pixel pitch is generally limited (commercial products show values around $1.5 \mathrm{~mm}$, although sensors with a pitch of $23.5 \mu \mathrm{m}$ have been published [144]). In addition, the operational bandwidth is reduced, covering mainly below $500 \mathrm{GHz}$ and high-power levels are required. In parallel, in electrooptic based $\mathrm{THz}$ systems (free space) it is possible to perform spatiotemporal measurements of the focal plane performing a 2D THz detection and launching the $800 \mathrm{~nm}$ signal modulated with $\mathrm{THz}$ information to an imaging systems such as a CCD (Charged Coupled Devices) [141].Camera-based systems tend to suffer from constant re-alignment of the optical source when 2D electro-optic systems are used and small dynamic range in THz cameras.

The alternative common approach is based on aperture scanning techniques, i.e. knife-edge, slit and pinhole [145]. Each of them has its own benefits and disadvantages, but their almost straightforward application makes their presence widespread in laboratories. The underlying idea is to scan a paraxial beam by stepwise blocking it with an opaque metallic screen with an aperture or edge that allows to pass only a small amount of radiation that reaches the detector. The pinhole and slit methods suffer of inevitable trade-off between the desired high spatial resolution, that is achieved by reducing the aperture size, and the transmitted intensity [145].

Firester et al [138] in 1978 described the knife edge (KE) method for optical wavelengths. It consists in measuring the power not occluded by the razor blade as it moves in a direction perpendicular to the beam propagation, by simultaneously recording its position with a power or energy meter. The amplitude of the unmasked radiation is plotted against the displacement in an error-function curve and finally the beam profile is retrieved by differentiating it with respect to the position. This last point could be a source of error since each fluctuation would be amplified, resulting in an inaccurate output [150]. Besides, mechanical movements suffer from irregularities and limitation, so that anomalies are inevitable. 
Later, a similar procedure was proposed based on a slit. It was theoretically studied by Mata-Mendez et al. 1983 [146], [147] and McCally et al. 1984 [148] who demonstrated the method by scanning a beam with a narrow slit, getting its gaussian profile. Mylonakis et al 2018 [149] studied the negative version of the actual aperture-based technique, since they obtained the optical beam profile by spanned it with a thin opaque round wire, a technique that could be employed as long as the shape of the beam is well-known. They took the ratio between the minimum and total output power and directly attained the beam profile. Fig. 36 depicts the three described aperture scanning techniques.

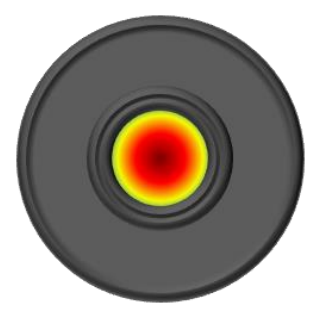

(a)

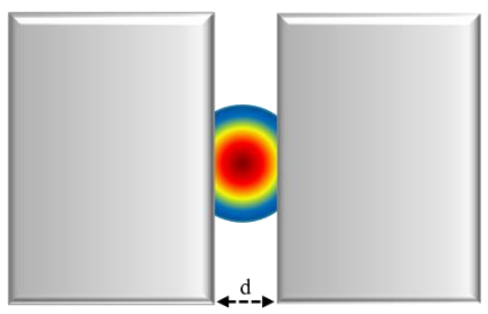

(b)

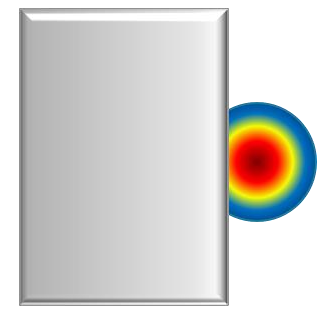

(c)

Fig. 36: Sketch of the most common aperture scanning methods (from left to right) the pinhole, slit and KE. The beam profile is recorded by progressively blocking part on the incoming wave and letting pass a small portion of the signal that reaches the detector .

These techniques originally developed for optical signals can be applied to Terahertz frequencies [150].

Thus, the KE can be used to characterize $\mathrm{THz}$ beams. This method is not limited by beam intensity, yet it requires aberration corrections when characterizing subwavelength no - paraxial beams with coupled spatial and temporal fields [151].

The slit and pinhole methods can be employed as well although more implementations of the latter can be found in the literature. Bitzer et al. 2007 [152] put a pinhole onto a gimbal-mounted terahertz mirror, that rotated with respect to a stationary detector. Molloy et al. 2011 [150] firstly imaged a THz beam by raster scanning it with a small circular aperture that crosses the beam path by means of mechanical translation stages in $\mathrm{x}$ and $\mathrm{y}$ directions. They partially mitigated the loss of intensity by experimentally choosing a suitable aperture dimension, but to locate the pinhole at the centre of the beam was still troublesome.

An alternative implementation of the pinhole can be achieved by swapping the moving and fixed elements. Jepsen et al. 1995 [153] scanned the terahertz beam generated by a large aperture photoconductive antenna with a similar detector hold on a translational stage. This implied the constant re-alignment of the system, 
that is generally not recommended in such sensitive configurations. Thirteen years later, Bitzer et al. 2008 [154] slightly adjusted this approach creating a periscope, i.e. not only the detector chip itself moved, but the entire detection unit, focusing lenses included. They were mounted on two separated translational stages and displaced with respect to the fixed elements of the set up. Elmabruk et al. 2019 [155] recalled the same principle and applied it in a fibre-based system. He moved the detection antenna and, since the optical path difference between the generation and detection arms of the setup were continuously changing, it was constantly compensated by the different time delays introduced by the optical delay line. The Hartmann test, an established technique mostly used to study aberrations in the primary mirrors of telescopes, was also employed to measure the profile of a wavefront in the terahertz band, in particular its deviation from the propagation axis [156].

All the methods presented so far rely on using a metal to spatially block the radiation in order to determine the spatial distribution of the beam. Here, we follow a different approach. What would happen if in pulsed $\mathrm{THz}$ system a dielectric is used to determine the spatial distribution? It can be hypothesized that it could help to overcome the loss of energy associated with blocking the beam. 


\subsection{Principle of operation}

Three basic assumptions of all aperture methods are that the incident beam is paraxial, there are no losses and the blocking structure is made of a perfect conductor [157]. Here, we depart from this last paradigm and, unlike previous schemes, the use of dielectric-based slit beam profiler for pulsed beam is researched. Similarly, other techniques such as KE and pinhole can be implemented.

In a dielectric beam profiler, the underlying mechanism is different from conventional metal-based schemes. For KE and certain slit and pinhole dimensions the effect of introducing two different dielectrics in a pulsed beam results in the split of the pulse in two temporal pulses each one associated to one spatial region (Fig. 37). Analysis of the Fourier spectra when the second small pulse appears shows that both spectra decay almost at the same frequency (Fig. 38).

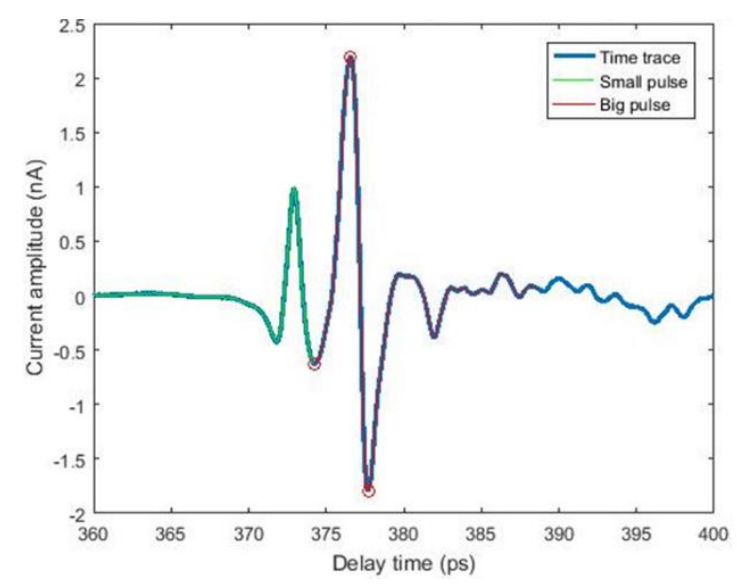

Fig. 37: Measurement of the split of the pulse into two temporal pulses due the presence of a 1 $\mathrm{mm}$ width dielectric slit.

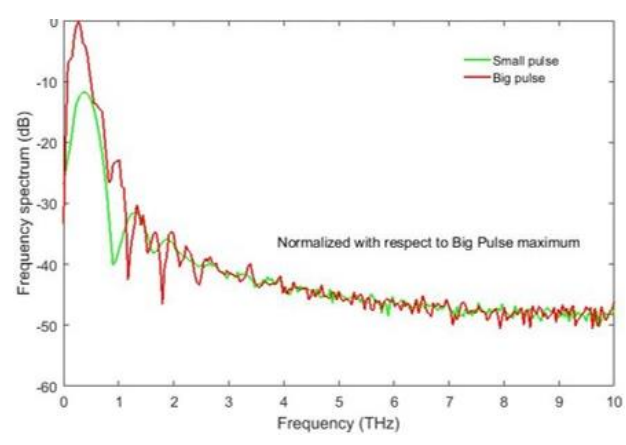

(a)

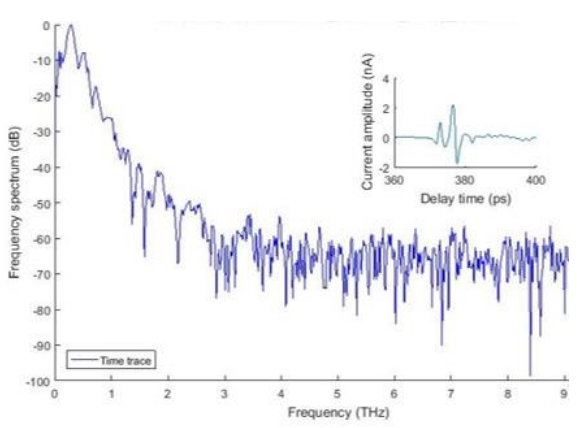

(b)

Fig. 38: a) Comparison of the Fourier spectra of the main and secondary pulses on the slit edge. In a) red line represents the frequency spectrum of the main pulse, whereas the green line corresponds to the delayed pulse. In b) the blue line represents the Fourier spectrum of the whole pulse. Inset: the temporal trace on the dielectric edge position. 
Thus, the temporal trace that is measured changes when the slit is swept across the beam. The energy of the pulse should be conserved if it is the only phenomenon involved. Thus, the impact of diffraction must be taken into account.

It was previously studied that high contrast images could be achieved by taking advantage on destructive interferences induced by diffraction where a dielectric edge of certain height was present [158]. In the Thesis, a dielectric slit is used to resolve spatial feature of the $\mathrm{THz}$ beam. For this reason, slits of different width, $a$, were considered. In every case, the width was narrower than the beam waist (see Fig. 39), which was estimated to be approximately $2 \mathrm{~mm}$ in the focus and $10 \mathrm{~mm}$ in the collimated areas.

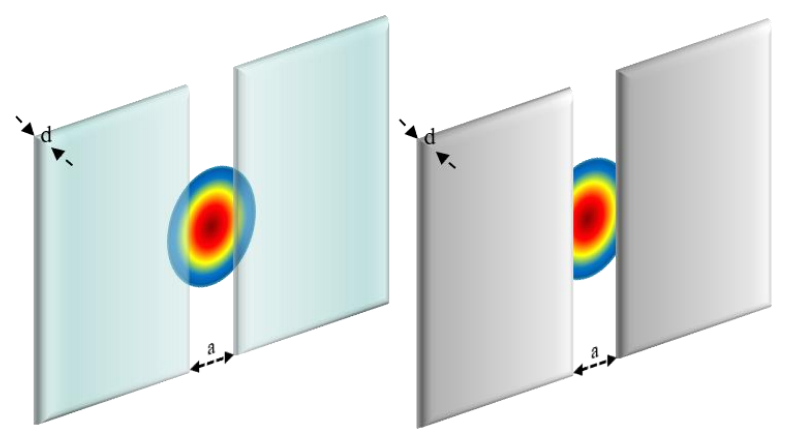

Fig. 39: Sketch of the dielectric and metal slit with an incident gaussian beam.

\subsection{Diffraction}

Diffraction has been studied to clarify its role in the dielectric-based beam profiler. The models detailed in Annex I were the starting points for the simulation of the behaviour of a plane wave incident on dielectric and metal edges. Models were adapted to describe the behaviour of slits, with slabs made of a dielectric as well as metallic slabs for the sake of comparison, with variable width and a fixed thickness $d$ of $1 \mathrm{~mm}$, at normal incidence $(\alpha=\pi / 2)$. The resulting field at a point $\mathrm{P}$ behind the screen was calculated as the sum of the field scattered by each edge. 


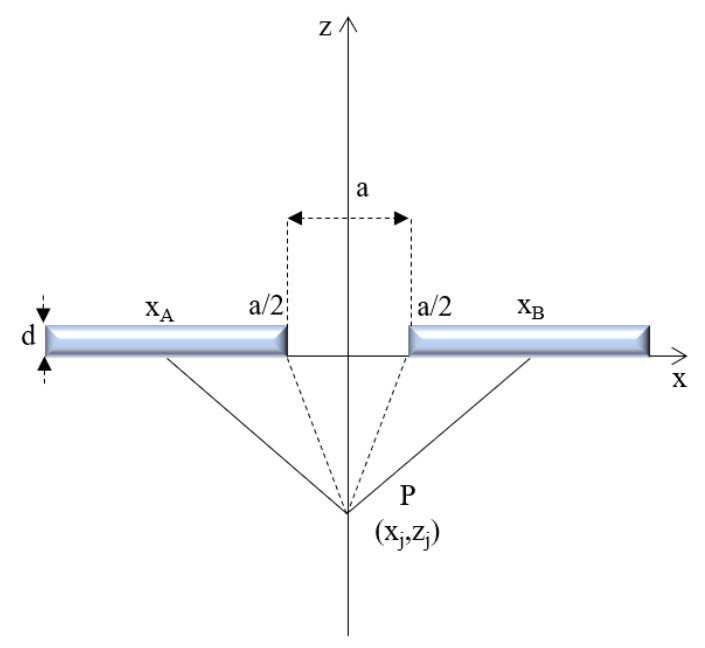

Fig. 40: Model of the slit simulation, both metal and dielectric.

For the dielectric model, the field can be approximated by:

$$
\begin{aligned}
& E_{d}(P)=E_{d A}+E_{d B} \\
& E_{d A}(P)=A_{0} e^{-i k R_{A} \cos \left(\theta_{A}-\alpha\right)}\left[\frac{1+e^{i \varphi}}{2}+\left(1-e^{i \varphi}\right) \frac{1-i}{\sqrt{2 \pi}} \int_{0}^{U_{A}} e^{i \mu^{2}} d \mu\right] \\
& E_{d B}(P)=A_{0} e^{-i k R_{B} \cos \left(\theta_{B}-\alpha\right)}\left[\frac{1+e^{i \varphi}}{2}+\left(1-e^{i \varphi}\right) \frac{1-i}{\sqrt{2 \pi}} \int_{0}^{U_{B}} e^{i \mu^{2}} d \mu\right]
\end{aligned}
$$

And for the metal model by:

$$
\begin{array}{r}
E_{m}(P)=E_{m A}+E_{m B} \\
E_{m A}(P)=A_{0} e^{-i k R_{A} \cos \left(\theta_{A}-\alpha\right)}\left[\frac{1}{2}+\frac{1-i}{\sqrt{2 \pi}} \int_{0}^{U_{A}} e^{i \mu^{2}} d \mu\right] \\
E_{m B}(P)=A_{0} e^{-i k R_{B} \cos \left(\theta_{B}-\alpha\right)}\left[\frac{1}{2}+\frac{1-i}{\sqrt{2 \pi}} \int_{0}^{U_{B}} e^{i \mu^{2}} d \mu\right]
\end{array}
$$

Where:

$$
\begin{aligned}
& R_{A}=\sqrt{(x+a / 2)^{2}+z^{2}}, R_{B}=\sqrt{(-x+a / 2)^{2}+z^{2}}, U_{A}=\sqrt{2 k R_{A} \sin \left(\frac{\theta_{A}-\alpha}{2}\right)}, \\
& U_{B}=\sqrt{2 k R_{B} \sin \left(\frac{\theta_{B}-\alpha}{2}\right)}
\end{aligned}
$$

This last model for the metal slit does not consider the edge thickness as a parameter of interest since previous studies proved that the edge parameters, height included, did not affect the final interference pattern [159]. However, in a metal slit, thickness could induce transmission losses if it behaves as a metal waveguide. Bromage et al., 1997 [160] highlighted the role of the thickness of a metal slit since it contributed to the shaping of the $\mathrm{THz}$ pulse. This parameter enabled the switch between two possible operational modes of the metal slit that 
have the same effect of filtering part of the spectral band of the incident beam. This process was described by equation:

$$
\beta(v)=\left\{\begin{array}{l}
\frac{\pi}{a} \sqrt{\left(\frac{v}{v_{c}}\right)^{2}-1}, v>v_{c} \\
i \frac{\pi}{a} \sqrt{1-\left(\frac{v}{v_{c}}\right)^{2}}, v \leq v_{c}
\end{array}\right.
$$

with $v_{c}=c / 2 a$ as the cut - off frequency, and $T(v)=e^{i \beta(v) d}$.

Two scenarios can be considered when:

- $v>v_{c}, \beta$ is real and the mode propagates through the waveguide (slit)

- $v \leq v_{c}\left(\lambda \geq \lambda_{c}\right), \beta$ is imaginary and the mode is evanescent

If $d \gg \lambda_{C}=2 a$, the power of the frequencies $v<v_{c}$ dramatically decreases converting the slit into a low pass filter in which frequencies higher than the cut off frequency would experience strong transmission losses and would not be transmitted. For example, a slit width of $0.05 \mathrm{~mm}$ will lead to a $v_{c}$ of $3 \mathrm{THz}$. Nevertheless, in the present work $\mathrm{THz}$ pulse signal is assumed to have energy mainly below $3 \mathrm{THz}$. Therefore, the only losses are due to diffraction, since it is not operating in the waveguide regime.

\subsection{Numerical simulation}

The proposed theoretical models were simulated to assess the performance of the beamprofiler. Simulations were made with a fixed thickness $d$ of $1 \mathrm{~mm}$, for normal incidence $(\alpha=\pi / 2)$ for frequencies from $0.1 \mathrm{THz}$ to $1 \mathrm{THz}$, with slit widths from 0.1 $\mathrm{mm}$ to $1 \mathrm{~mm}$, with $0.1 \mathrm{~mm}$ step. Dielectric refractive index was chosen to be 1.6 since measurements were performed with PMMA. Fig. 41 collects simulations of a THz pulse as it approaches and crosses a metal slit (Fig. 41 a) and c) top and side view) and a dielectric slit of $0.5 \mathrm{~mm}$-width (Fig. $41 \mathrm{~b}$ ) and d) top and side view), following the model described in section 4.3. Comparing these results with the theory exposed in Annex I, it can be clearly noticed the effect of diffraction in the dielectric slit as $d$-waves (see Annex I) are generated with the characteristic parabola shape, one per each edge and the resulting interference pattern is shown. These studies will be useful to define the potential of the dielectric slabs as beam profilers, since the interference pattern in the region behind the slit will determine the energy available for the beam scanning. 
METAL

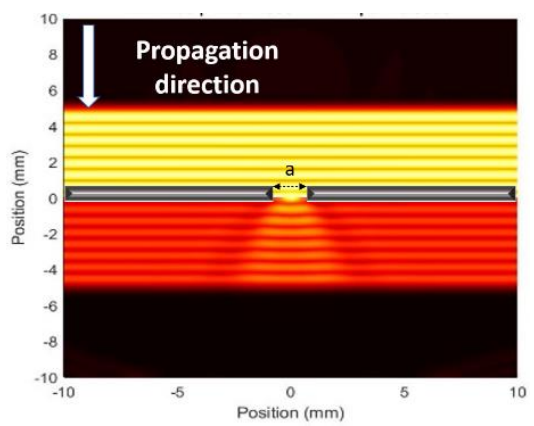

(a)

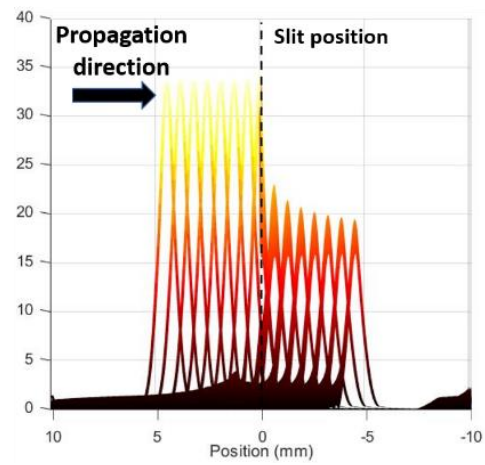

(c)
DIELECTRIC

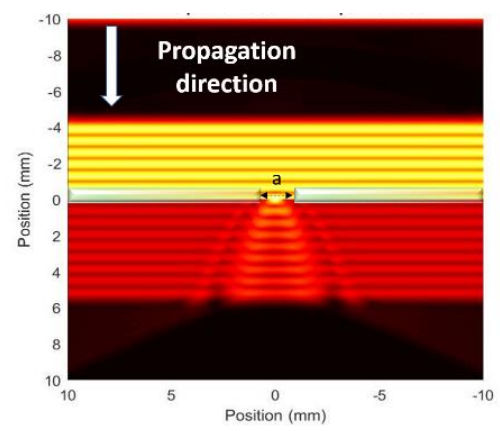

(b)

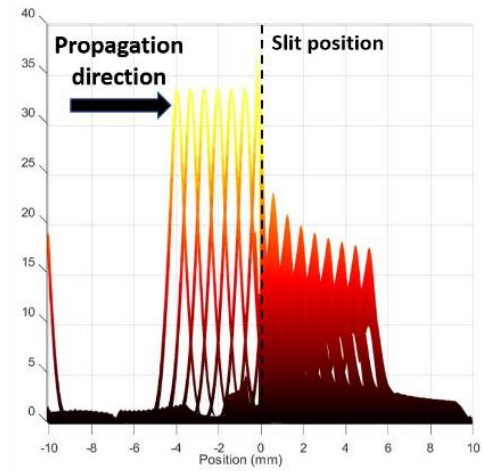

(d)

Fig. 41: Results of the simulation of the sum of $\mathrm{THz}$ as it propagates through a slit with an aperture of $0.5 \mathrm{~mm}$-width. Top view of the propagation of the resulting pulse for the metal (a) and dielectric slit (b) and evolution of the THz pulse across a line parallel to the $y$-axis (c), (d). 
METAL

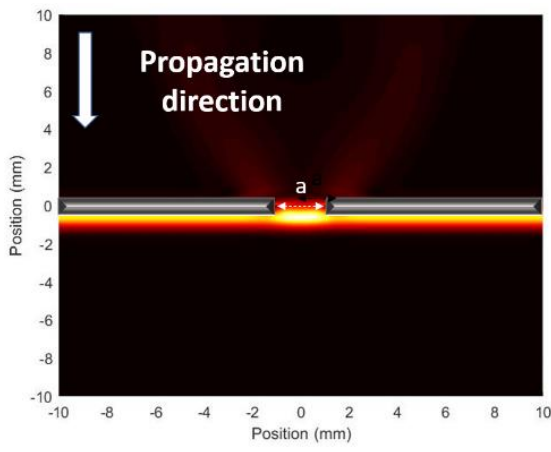

(a)

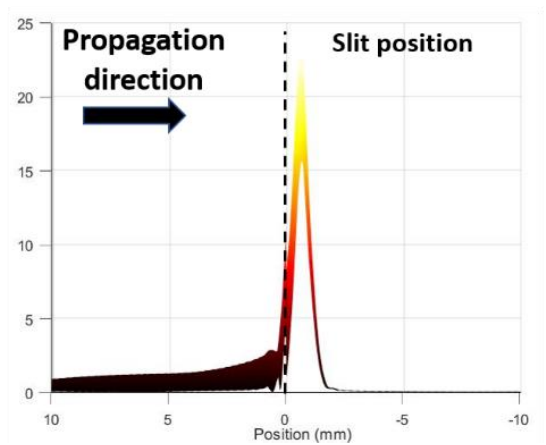

(c)

\section{DIELECTRIC}

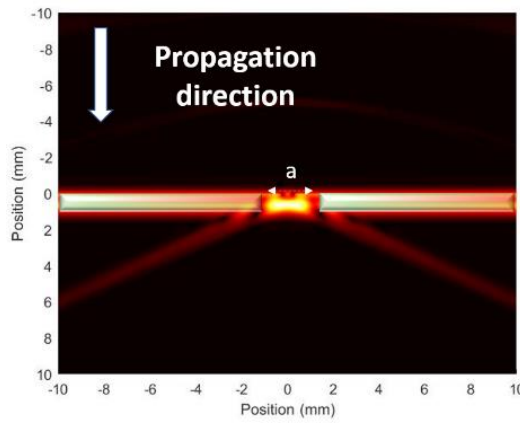

(b)

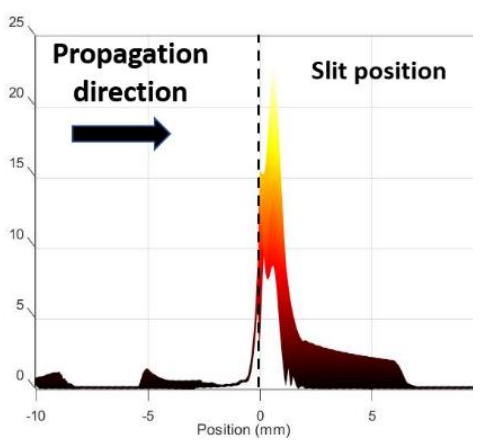

(d)

Fig. 42: Results of the simulation of a single THz pulse just behind the slit of $0.5 \mathrm{~mm}$ width. Top view of the propagation of the resulting wave for the metal (a) and dielectric slit (b) and evolution of the THz pulse across a line parallel to the $y$-axis (c), (d).

If a closer look is given to one specific pulse, simulations show that the dielectric slit splits the main pulse into two smaller pulses, as experimentally verified (see Fig. 37 and Fig. 42 from a) to d)).

As previously described, one of the effects of the dielectric slit is to split the main pulse into two. Thus, frequency - wise the dielectric slit is inducing a filter response in which part of the frequencies are attenuated. Finally, in order to assess the quantitative differences with the two methods, simulations were performed to assess the slit behaviour in the frequency domain, as the dielectric slit acts as a filter depending on its width, on the slab thickness and the refractive index they are made of. 


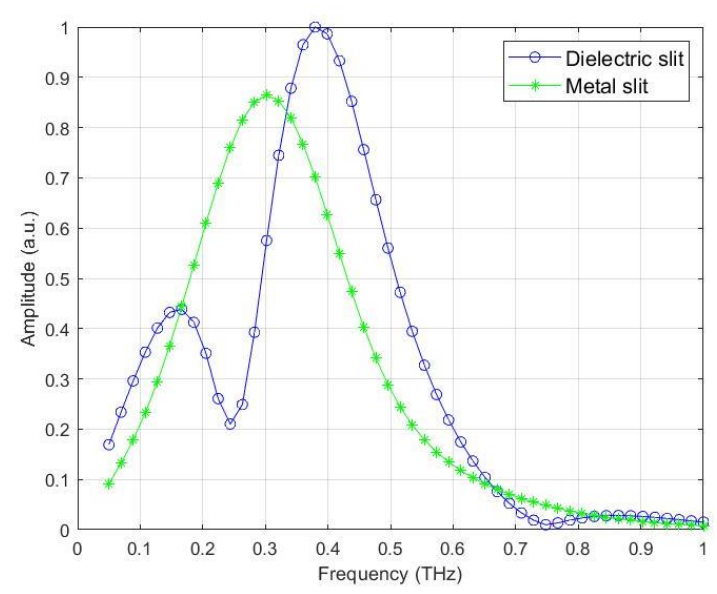

Fig. 43: Comparison of the contribution of each frequency to the field after having crossed the slit for an aperture of $0.5 \mathrm{~mm}$.

As displayed in Fig. 43, a different behaviour is expected for slits created from both materials, where the output amplitude has been normalized to the maximum output power of the pulse after the dielectric slit. In the case of the dielectric slit, it is clearly noticeable that the signal has been filtered. At $0.24 \mathrm{THz}$, the amplitude is strongly suppressed, due to the destructive interference induced by the step phase related to slab thickness (see Eq. 13). Furthermore, it is interesting to point out that frequencies above approximately $0.34 \mathrm{THz}$ show higher fields values after the dielectric slit than in the metal one due to the different nature of the beamprofiling method.

\subsection{Experimental results}

The concept of using dielectric materials for beamprofiling has been experimentally explored. The profile of a THz beam from a pulsed THz-TDS system has been measured using dielectric slabs (PMMA, refractive index of 1.6) to form a slit and these results compared to the conventional metallic-based slit (Aluminium). Fig. 44 shows the beam profile of a pulsed THz beam measured with a slit width of approximately $0.4 \mathrm{~mm}$. The beam profiler is placed in the second collimation area, that is between L3 and L4 (see Fig. 3 a). Similar values of beam waist, 1/e, are achieved with both methods showing the feasibility of the technique. The measured beam waist is $1.05 \mathrm{~mm}$ for the metal and $0.9 \mathrm{~mm}$ for the dielectric slit. It can be noticed that the intensity of the beam profile is higher for the dielectric slit than for the metal one due to the different nature of the approach. 


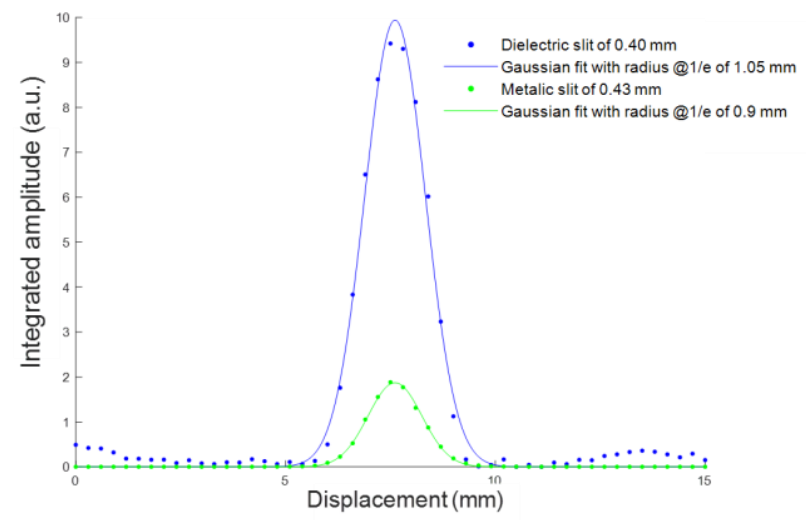

Fig. 44: Beam profile of the THz pulse measured with both metal (green dots) and dielectric (blue dots) slits. For both cases, the profiles are fitted with the gaussian shape and the radius at $1 / \mathrm{e}$ is calculated.

A study has been performed to measure the dependence of the signal amplitude as a function of the slit width. It can be seen that, as shown in Fig. 45 , the dielectricbased slit allows the measurement of higher currents.

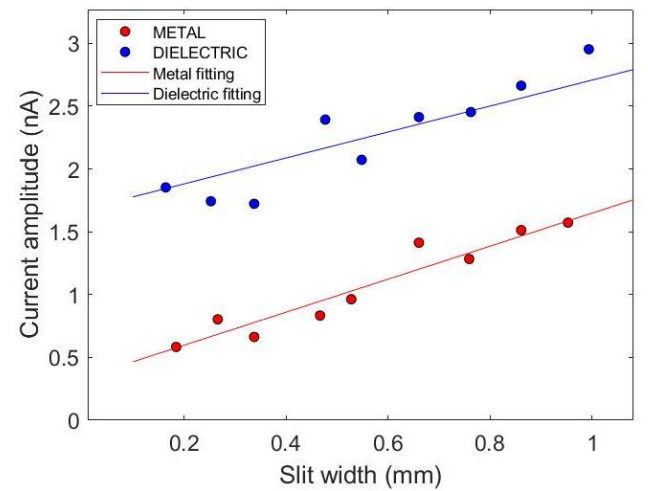

Fig. 45: Experimental results achieved by measuring the maximum peak current of the $\mathrm{THz}$ beam as the profiler moves perpendicular to the propagation axis.

As an additional test, the radiation pattern of the PCA emitter antenna has been measured to compare the performance of the conventional metallic slit and the one based on dielectric slabs. The detected pattern exhibits similarities with the radiation pattern of the PCA emitter (See Fig 4 of [153]). Fig. 46 compares the results achieved with slits of both materials with an aperture of approximately 0.45 $\mathrm{mm}$. 


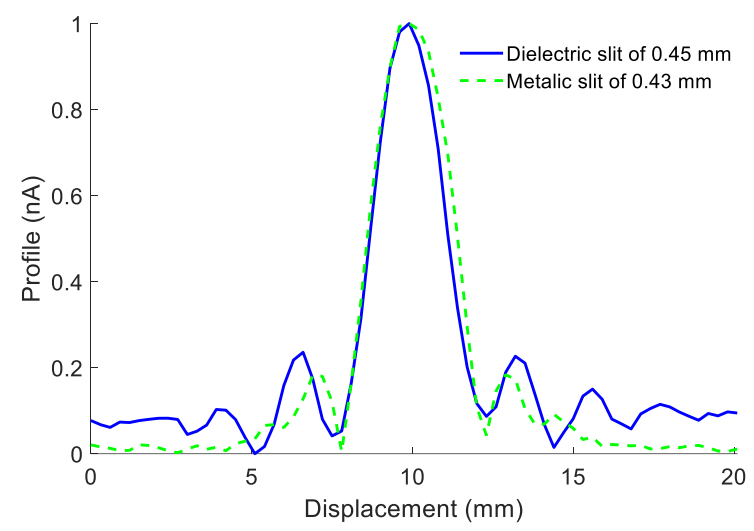

Fig. 46: Experimental results of the measure of the radiation pattern of emission of the emitter. Measurements with the total integral intensity suggested that a dielectric slit distinguishes more orders of secondary lobes and with slightly higher amplitude at a specific frequency of $0.3 \mathrm{THz}$.

As it can be noticed, both methods show a similar beam pattern. Even, the dielectric slit seems to be able to enable the detection of one more order of secondary lobes, with even a slightly higher amplitude. 


\subsection{Conclusion}

The ability of a dielectric slit to act as a beam profiler was investigated and compared with the standard method.

Simulations and measurements suggested that the proposed solution could perform well for the study of THz spatial features. Nevertheless, additional work is needed to further validate the method and extrapolate to other wavelength regimes. Other apertures could be designed, such as KE and pinhole to research their behaviour when made of dielectric material. Finally, measurements with dielectric materials with different refractive indexes and thicknesses should be performed, as to set the optimum geometrical parameters to take advantage on the diffraction. 
4. Dielectric - based beam profiler 
The importance of the T-rays for plenty of fields and applications has been described and motivated throughout the Thesis and laid the ground for the researched work that was carried out. Measurements have been performed with an all-fibre THz-TDS system with two photoconductive antennas, that operate at the $1.5 \mu \mathrm{m}$, which allowed to make use of the excellent properties of the fibres in this band. Researches gathered in the present work have been performed as to foster the use of the $\mathrm{THz}$ and to deepen in their knowledge. For this reason, the system has been used to firstly investigate the potential of alternative passive devices, in particular polarizers, to discriminate the polarization state of an incoming wave by using ordinary low-cost materials such as paper and graphite. Its absorption properties enabled dichroism effect and the performance result hint at the possibility of using such a device as a polarizer.

Subsequently, another cost-effective approach has been presented to rapidly achieved flexible and inexpensive polarizers. In this case, EGaln ${ }_{24.5}$ alloy proved to be an efficient, low-cost and no toxic conductive ink that allowed direct drawing of $\mathrm{THz}$ polarizers on flexible substrates (PVA), paving the way to its deposition by means of ad-hoc modified printers. In this way, repeatability and bandwidth could be better controlled, leading to improved performance of the final fabricated device. In fact, sub-THz wire grid polarizers were finally achieved, by selectively deploying the alloy on PVC substrate. These researches aimed to provide rapid and inexpensive methods to laboratories in order to be able to quickly discriminate 
measurement states of electromagnetic waves. The remarkable improvements in additive fabrication processes and their low-cost implementation are paving the way for their use in scientific applications, far beyond the fabrication of holder and bases for optical elements. Technology is almost mature enough to enable the printing of more complex passive components. In the next future it could be possible to print a sub millimetre $\mathrm{THz}$ polarizer, using a material with the proper electrical conductivity, avoiding the addition of metals such as gold or copper.

As previously pointed out, $\mathrm{THz}$ are currently gaining presence in several industrial fields, since they turned out to be extremely appealing, thanks to their transparency to ordinary materials, such as fabrics, polymers and cardboards, that are mostly used as packaging. Therefore, terahertz waves raised interest as a potential tool for inline quality control equipment. In this work, the potential of a fixed-delay configuration for the characterization of defects through $\mathrm{THz}$ waves, avoiding the need to record the entire $\mathrm{THz}$ trace has been presented. Measurement time was reduced, and valuable information were extracted. Further work should be done to test the method in relevant environments, since this technique could be of interest in some industries to replace more complex tools. A more general approach could be investigated to apply the proposed technique to the identification of defects of different nature, both on the surface and inside a specific piece or industrial part. regardless the defect shape or material. Furthermore, it could be considered to substitute the current Gaussian beam with a cylindrical one, similar to a fan beam that would enable a single trace - single measurement.

Finally, a method to deepen in the THz knowledge was investigated. The most popular scanning method currently available (camera-based and aperture scanning systems) exhibited both advantages and shortcomings, mainly related with the inevitable trade-off between their spatial resolution and the amount of energy that reached the detector. To overcome this issue, the use of a dielectric beam profiler was proposed and analysed. Simulations and measurements suggested that the proposed solution could perform well for the study of $\mathrm{THz}$ spatial features. Nevertheless, additional work is needed to further validate the method and extrapolate to other wavelength regimes.

To sum up, passive dielectric-based devices and a technique to check dielectric components have been proposed as to increase $\mathrm{THz}$ component affordability and availability, as well as methods to make $\mathrm{THz}$ closer to the industrial, world. 
Dielectric-based Components and Methods for Terahertz Sensing 
5. Conclusion 


\section{Bibliography}

[1] Y. S. Lee, Principles of Terahertz Science and Technology, third ed. ed., Springer US, 2009, pp. 259 - 294.

[2] S. L. Dexheimer, Terahertz spectroscopy, principle and applications, New york: University of Rochester, 2008.

[3] D. W. Faries, K. A. Gehring, P. L. Richards and Y. R. Shen, "Tunable Far-Infrared Radiation Generated from the Difference Frequency between two Ruby Lasers," Physical review, vol. 180, no. 2, pp. 363 - 365, 1969.

[4] K. H. Yang, P. L. Richards and Y. R. Shen, "Generation of Far-Infrared Radiation by Picosecond Light Pulses in LiNbO3," Appl. Phys. Lett., vol. 19, no. 320, 1971.

[5] L. Duvillaret, F. Garet, J.-F. Roux and J.-L. Coutaz, "Analytical Modeling and Optimization of Terahertz Time-Domain Spectroscopy Experiments Using Photoswitches as Antennas," IEEE J. Sel. Top. Quant., vol. 7, no. 4, pp. 615 - 623, 2001.

[6] R. B. Kohlhaas, S. Breuer, S. Nellen, L. Liebermeister, M. Schell, M. P. Semtsiv, W. T. Masselink and B. Globisch, "Photoconductive terahertz detectors with $105 \mathrm{~dB}$ peak dynamic range made of rhodium doped InGaAs," Appl. Phys. Lett., vol. 114, no. 221103, pp. 1 - 5, 2019.

[7] K. -. E. Peiponen, J. A. Zeitler and M. Kuwata - Gonokami, Terahertz spectroscopy and imaging, USA: Springer series in optical science, 2013.

[8] K. Fujita, S. Hayashi, A. Ito, M. Hitaka and T. Dougakiuchi, "Sub-terahertz and terahertz generation in long-wavelength quantum cascade lasers," Nanophotonics, vol. 8, no. 12, pp. 2235 - 2241, 2019.

[9] R. A. Lewis, "A review of terahertz detectors," J. Phys. D: Appl. Phys., vol. 52, no. 433001, pp. 1 - 23, 2019.

[10] Q. Wu and C. Zhang, "Free-space electro-optic sampling of terahertz beams," Appl. Phys. Lett., vol. 67, no. 3523, 1995.

[11] P. U. Jepsen, C. Winnewisser, M. Schall, V. Shyja, S. R. Keiding and H. Helm, "Detection of THz pulses by phase retardation in lithium tantalate," Phys. Rev. E, vol. 53, no. 4, pp. R3052 - R3055, 1996.

[12] A. Nahata, D. H. Auston, T. F. Heinz and C. Wu, "Coherent detection of freely propagating terahertz radiation by electro-optic sampling," Appl. Phys. Lett., vol. 68, no. 150, 1996.

[13] D. H. Auston and P. R. Smith, "Picosecond optical electronics: new approaches to high speed electronics instrumentation," CLEO Conference on Lasers and Electro-Optics, p. WO1, 1982. 
[15] P. L. Richards, "Bolometers for infrared and millimeter waves," J. Appl. Phys., vol. 76, no. 1, 1994.

[16] P. U. Jepsen, D. G. Cooke and M. Koch, "Terahertz spectroscopy and imaging - Modern techniques and applications," Laser Photonics Rev., vol. 5, no. 1, pp. 124 - 166, 2011.

[17] G. J. Wilmink, B. L. Ibey, B. D. Rivest, J. E. Grundt, W. P. Roach, T. D. Tongue, T. D. Schulkin, N. Laman, X. G. Peralta, C. C. Roth and C. Z. Cerna, "Development of a compact terahertz time-domain spectrometer for the measurement of the optical properties of biological tissues," J. Biomed. Opt., vol. 16, no. 047006, 2011.

[18] M. A. Báexz-Chorro and B. Vidal, "Single trace terahertz spectroscopic ellipsometry," Opt. Express, vol. 27, no. 24, pp. 35468 - 35474, 2019.

[19] Y. Kim and D.-S. Yee, "High-speed terahertz time-domain spectroscopy based on electronically controlled optical sampling," Opt. Lett., vol. 35, no. 22, pp. 3715 - 3717, 2010.

[20] M. Yahyapour, A. Jahn, K. Dutzi, T. Puppe, P. Leisching, B. Schmauss, N. Vieweg and A. Deninger, "Fastest Thickness Measurements with a Terahertz Time-Domain System Based on Electronically Controlled Optical Sampling," Appl. Sci., vol. 9, no. 1283, pp. 1 -11, 2019.

[21] N. Krumbholz, T. Hochrein, N. Vieweg, T. Hasek, K. Kretschme, M. Bastian, M. Mikulics and M. Koch, "Monitoring polymeric compounding processes inline with $\mathrm{THz}$ time-domain spectroscopy," Polym. Test, vol. 28, no. 1, pp. 30 -35, 2009.

[22] A. S. Bockelt, B. Vidal and J. Palací, "All-fiber centralized architecture for parallel terahertz sensors," IEEE trans on Terahertz Sci. and Technol., vol. 5, no. 1, pp. 137 - 144, 2015.

[23] N. M. Burford and M. O. El-Shenawee, "Review of terahertz photoconductive antenna technology," Opt. Eng., vol. 56, no. 1, pp. 1 - 20, 2017.

[24] A. Bockelt and B. Vidal, "Dynamic tuning of mean carrier momentum relaxation time in InGaAs/InAlAs THz photoconductive switches," IEEE Trans on Terahz Sci. and Tech., vol. 7, no. 1, pp. 107 - 113, 2017.

[25] S. Lepeshov, A. Gorodetsky, A. Krasnok, E. Rafailov and P. Belov, "Enhancement of terahertz photoconductive antenna operation by optical nanoantennas," Laser Photonics Rev, vol. 11, no. 1, pp. 1 - 21, 2017.

[26] E. Castro - Camus and M. Alfaro, "Photoconductive devices for terahertz pulsed spectroscopy: a review (Invited)," Photonics Res., vol. 4, no. 3, pp. A36 - A42, 2016.

[27] J. Rudd and D. Mittleman, "Influence of substrate-lens design in terahertz time-domain spectroscopy," J. Opt. Soc. Am. B, vol. 19, no. 2, pp. 319 - 329, 2001.

[28] B. Sartorius, H. Roehle, H. Künzel, J. Böttcher, M. Schlak, D. Stanze, H. Venghaus and M. Schell, "All-fiber terahertz time-domain spectrometer operating at $1.5 \mu \mathrm{m}$ telecom wavelengths," Opt. Express, vol. 16, no. 13, pp. 9565 - 9570, 2008.

[29] S. S. Dhillon, M. S. Vitiello, E. H. Linfield, A. G. Davies, M. C. Hoffmann, J. Booske, C. Paoloni, M. Genshc, P. Weightman, G. P. Williams, E. Castro-Camus, D. R. S. Cumming, F. Simoens, I. Escorcia-Carranza, J. Grant, S. Lucyszyn, M. Kuwata-Gonokami, K. Konishi, M. Koch, C. A. Schmuttenmaer, T. L. Cocker, R. Huber, A. G. Markelz, Z. D. Taylor, V. P. Wallace, J. A. Zeitler, J. Sibik, T. M. Korter, B. Ellison, S. Rea, P. Goldsmith, K. B. Cooper, R. Appleby, D. Pardo, P. G. Huggard, V. Krozer, H. Shams, M. Fice, C. Reanud, A. Seeds, A. Stöhr, M Naftaly, N. Ridler, R. Clarke, J. E. Cunningham and M. B. Johnston, "The 2017 terahertz science and technology roadmap," J. Phys. D Appl. Phys., vol. 50, no. 043001, pp. 1 - 49, 2017.

[30] R. J. Falconer and A. G. Markelz, "Terahertz Spectroscopic Analysis of Peptides and Proteins," J. Infrared Milli. Terahz. Waves, vol. 33, p. 973-988, 2012.

[31] X. Li, T. Qiu, J. Zhang, E. Baldini, J. Lu, A. M. Rappe and K. A. Nelson, "Terahertz fieldinduced ferroelectricity in quantum paraelectric SrTiO3," Science, vol. 364, no. 6445, pp. 1079-1082, 2019 
[32] J. Neu, D. J. Aschaffenburg, M. R. C. Williams and C. A. Schmuttenmaer, "Optimization of Terahertz Metamaterials for Near-Field Sensing of Chiral Substances," IEEE Trans. Terahertz Sci. Technol., vol. 7, no. 6, pp. 755-764, 2017.

[33] M. Brucherseifer, M. Nagel, P. Haring Bolivar and H. Kurz, "Label-free probing of the binding state of DNA by time-domain terahertz sensing," Appl. Phys. Lett, vol. 77, no. 24, pp. 4049 - 4051, 2000

[34] K. Ajito and Y. Ueno, "THz Chemical Imaging for Biological Applications," IEEE T. THz Sci. Techn., vol. 1, no. 1, pp. 293 - 300, 2011.

[35] C. Yu, S. Fan, Y. Sun and E. Pickwell-MacPherson, "The potential of terahertz imaging for cancer diagnosis: A review of investigations to date," Quant Imaging Med Surg., vol. 2, no. 1, pp. 33 - 45, 2012.

[36] C. Kulesa, "Terahertz Spectroscopy for Astronomy: From Comets to Cosmology," IEEE T. THz Sci. Techn., vol. 1, no. 1, pp. 232 - 240, 2011.

[37] H. Hoshina, Y. Sasaki, A. Hayashi, C. Otani and K. Kawase, "Noninvasive Mail Inspection System with Terahertz Radiation," Appl. Spectrosc., vol. 63, no. 1, pp. 1 - 7, 2009.

[38] J. Jansen, S. Wietzke and M. Koch, "Terahertz Spectroscopy of Polymers," in Terahertz Spectroscopy and Imaging, Springer, 2013.

[39] C. D. Stoik, M. J. Bohn and J. L. Blackshire, "Nondestructive evaluation of aircraft composites using transmissive terahertz time domain spectroscopy," Opt. Express, vol. 16, no. 21, pp. 17039 - 17051, 2008.

[40] Q. Wang, X. Li, T. Chang, J. Zhang, L. Liu, H. Zhou and J. Bai, “Nondestructive imaging of hidden defects in aircraft sandwich composites using terahertz time-domain spectroscopy," Infrared Phys. Technol., vol. 97, pp. 326 - 340, 2019.

[41] C.-H. Ryu, S.-H. Park, D.-H. Kim, K.-Y. Jhang and H.-S. Kim, "Nondestructive evaluation of hidden multi-delamination in a glass-fiber-reinforced plastic composite using terahertz spectroscopy," Compos. Struct, vol. 156, pp. 338 - 347, 2016.

[42] J. Wang, J. Zhang, T. Chang, L. Liu and H.-L. Cui, "Terahertz nondestructive imaging for foreign object detection in glass fibre reinforced polymer composite panels," Infrared Phys. Technol., vol. 98, pp. 36 - 44, 2019.

[43] M. Báez-Chorro and B. Vidal, "Terahertz Spectroscopy of Engineered Stone," 44th Internation Conference on Infrared, Millimeter and Terahertz Waves (IRMMW-THz), Paris (France), 1 - 6 September 2019.

[44] S. Wietzke, C. Jansen, F. Rutz, D. M. Mittleman and M. Koch, "Determination of additive content in polymeric," Polym. Test., vol. 26, p. 614-618, 2007

[45] C. Jördens, M. Scheller, S. Wietzke, D. Romeike, C. Jansen, T. Zentgraf, K. Wiesauer, V. Reisecker, and M. Koch, "Terahertz spectroscopy to study the orientation of glass fibres in reinforced plastics," Compos. Sci. Technol, vol. 70, p. 472-477, 2010.

[46] D.-H. Han and L.-H. Kang, "Nondestructive evaluation of GFRP composite including multi-delamination using THz spectroscopy and imaging," Compos. Struct., vol. 185, pp. $161-175,2018$.

[47] M. Baéz-Chorro, R. Llorens-Chiralt and B. Vidal, "Comparison of THz Spectroscopy Measurements of Carbon Nanotubes Embedded in Polymer Matrices," 44th International Conference on Infrared, Millimeter and Terahertz Waves (IRMMW-THz), Paris (France), 1 6 September 2019.

[48] S. Krimi, J. Klier, M. Hermann, J. Jonuscheit and R. Beigang, "Inline multilayer thickness sensing by using terahertz time-domain spectroscopy in paint film," 38th Int. Conference on Infrared Millimeter and Terahertz Waves, 2013.

[49] W. Tu, S. Zhong, Shen, Y. Shen and A. Incecik, "Nondestructive testing of marine protective coatings using terahertz waves with stationary wavelet transform," Ocean Eng., vol. 111, pp. 582-592, 2016

[50] K. Su, Y.-C. Shen and J. A. Zeitler, "Terahertz Sensor for Non-Contact Thickness and Quality Measurement of Automobile Paints of Varying Complexity," IEEE T. THz Sci. Techn., vol. 4, no. 4, pp. 432-439, 2014. 
[51] F. Sanjuan, A. Bockelt and B. Vidal, "Determination of refractive index and thickness of a multilayer structure with a single $\mathrm{THz}$ time domain spectroscopy measurement," Appl. Opt., vol. 53, no. 22, pp. 4910 - 4913, 2014.

[52] G. Ok, J. S. Shin, M.-C. Lim and S.-W. Choi, "Large-scan-area sub-terahertz imaging system for nondestructive food," Food Control, pp. 383-389, 2019.

[53] Y. Jiang, H. Ge and Y. Zhang, "Detection of foreign bodies in grain with terahertz reflection imaging," Optik, vol. 181, pp. 1130 - 1138, 2019.

[54] K. Ahi, S. Shahbazmohamadi y N. Asadizanjani, «Quality control and authentication of packaged integrated circuits using enhanced-spatial-resolution terahertz time-domain spectroscopy and imaging," OPT. LASER ENG., vol. 104, pp. 274 - 284, 2018.

[55] B. B. Hu and M. C. Nuss, "Imaging with terahertz waves," Opt. Lett., vol. 20, no. 16, pp. 1716 - 1719, 1995.

[56] W. L. Chan, J. Deibel and D. M. Mittleman, "Imaging with terahertz radiation," Rep. Prog. Phys., vol. 70, pp. 1325 - 1379, 2007.

[57] Z. Long, T. Wang, C. You, Z. Yang, K. Wang and J. Liu, "Terahertz image super-resolution based on a deep convolutional neural network," Appl. Opt., vol. 58, no. 10, pp. 2731 - 2735, 2019.

[58] N. S. Balbekin, M. S. Kulya, A. V. Belashov, A. Gorodetsky and N. V. Petrov, "Increasing the resolution of the reconstructed image in terahertz pulse time-domain holography," Sci. Rep., vol. 9, no. 180, pp. 1 - 9, 2019.

[59] K. Ahi, "A method and system for enhancing the resolution of terahertz imaging," Measurement, vol. 138, pp. 614 - 619, 2019.

[60] H. Zhong, J. Xu, X. Xie, T. Yuan, R. Reightler, E. Madaras and X. C. Zhang, "Nondestructive defect identification with terahertz time-of-flight tomography," IEEE Sensors Journal, vol. 5, no. 2, pp. 203 - 208, 2005.

[61] C. C. Homes, G. L. Carr, R. P. Lobo, J. D. LaVeigne and D. B. Tanner, "Silicon beam splitter for far-infrared and terahertz spectroscopy," Appl. Phys. Lett, vol. 46, no. 32, pp. 7884 7888, 2007.

[62] B. S.-Y. Ung, C. Fumeaux, H. Lin, B. M. Fischer, B. W.-H. Ng and D. Abbott, "Low-cost ultra-thin broadband terahertz beam-splitter," Opt. Express, vol. 20, no. 5, pp. 4968-4978 2012.

[63] B. S.-Y. Ung, B. Weng, R. Shepherd, D. Abbott and C. Fumeaux, "Inkjet printed conductive polymer-based beam-splitters for terahertz applications," Opt. Mater. Express, vol. 3, no. 9, pp. 1242-1249, 2013.

[64] M. Nagai, N. Mukai, Y. Minowa, M. Ashida, J. Takayanagi and H. Ohtake, "Achromatic THz wave plate composed of stacked parallel metal plates," Opt. Lett., vol. 39, no. 1, pp. 146 - 149, 2014.

[65] B. Scherger, M. Scheller, N. Vieweg, S. T. Cundiff and M. Koch, "Paper terahertz wave plates," Opt. Express, vol. 19, no. 25, pp. 24884-24889, 2011.

[66] S.-H. Lee, S. Gee, C. Kang and C.-S. Kee, "Terahertz wave transmission properties of metallic periodic structures printed on a photo-paper," J. Opt. Soc. Korea, vol. 14, no. 3, pp. 282 - 285, 2010.

[67] T. Kondo, T. Nagashima and M. Hangyo, "Fabrication of wire-grid-type polarizers for THz region using a general-purpose color printer," Jpn. J. Appl. Phys., vol. 42, no. 4A, pp. L 373-L 375, 2003.

[68] M. Walther, A. Ortner, H. Meier, U. Löffelmann, P. J. Smith and J. G. Korvink, "Terahertz metamaterials fabricated by inkjet printing," Appl. Phys. Lett., vol. 95, no. 251107, pp. 1 3, 2009.

[69] A. Siemion, A. Siemion, M. Makowski, J. Suszek, J. Bomba, A. Czerwiński, F. Garet, J.-L. Coutaz and M. Sypek, "Diffractive paper lens for terahertz optics," Opt. Lett., vol. 37, no. 20, pp. 4320-4322, 2012.

[70] F. Sanjuan, A. Bockelt and B. Vidal, "Birefringence measurement in the $\mathrm{THz}$ range based on double Fourier analysis," Opt. Lett., vol. 39, no. 4, pp. 809 - 812, 2014. 
[71] E. Castro - Camus and M. B. Johnston, "Extraction of the anisotropic dielectric properties of materials from polarization-resolved terahertz time-domain spectra," J. Opt. A: Pure Appl. Opt., vol. 11, p. 105206-105212, 2009.

[72] C. M. Morris, R. Valdés Aguilar, A. V. Stier and N. P. Armitage, "Polarization modulation time-domain terahertz polarimetry," Opt. Express, vol. 20, no. 11, pp. 12303 - 12317, 2012.

[73] F. Sanjuan, G. Gaborit and J.-L. Coutaz, "Full electro-optic terahertz time-domain spectrometer for polarimetric studies," Appl. Opt., vol. 57, no. 21, pp. 6055 - 6060, 2018.

[74] D. J. Aschaffenburg, M. R. C. Williams and C. A. Schuttenmaer, "Terahertz spectroscopic polarimetry of generalized anisotropic media composed of Archimedean spiral arrays: Experiments and simulations," J. Chem. Phys, vol. 144, no. 174705, pp. 1 - 11, 2016.

[75] V. Yurchenko, J. A. Murphy, J. Barton, J. Verheggen and K. Rodgers, "Dual-Layer Frequency-Selective Grid Polarizers on Thin-Film Substrates for THz Applications," 38th European Microwave Conference, pp. 1014 - 1017, 2008.

[76] L. Ren, C. L. Pint, L. G. Booshehri, D. W. Rice, X. Wang, D. J. Hilton, K. Takeya, I. Kawayama, M. Tanouchi, R. H. Hauge and J. Kono, "Carbon nanotube terahertz polarizer," Nano Lett., vol. 9, no. 7, pp. 2610-2613, 2009.

[77] L. Ren, C. L. Pint, T. Arikawa, K. Takeya, I. Kawayama, M. Tonouchi, R. H. Hauge and J. Kono, "Broadband terahertz polarizers with ideal performance based on aligned carbon nanotube stacks," Nano Lett., vol. 12, no. 2, pp. 787-790, 2012.

[78] J. Kyoung, E. Y. Jang, M. D. Lima, H. -. R. Park, R. Ovalle Robles, X. Lepró, Y. H. Kim, R. H. Baughman and D. -. S. Kim, "A reel-wound carbon nanotube polarizer for terahertz frequencies," Nano Lett., vol. 11, no. 10, pp. 4227-4231, 2011.

[79] D. Polley, A. Ganguly, A. Barman and R. K. Mitra, "Polarizing effect of aligned nanoparticles in terahertz frequency region," Opt. Lett., vol. 38, no. 15, p. 188660, 2013.

[80] G. Postiglione, G. Natale, G. Griffini, M. Levi and S. Turri, "Conductive 3D microstructures by direct 3D printing of polymer/carbon nanotube nanocomposites via liquid deposition modeling," Compos. Part A Appl. Sci. Manuf., vol. 76, pp. 110 - 114, 2015.

[81] D. J. Dummer, S. G. Kaplan, L. M. Hanssen, A. S. Pine and Y. Zong, "High-quality Brewster's angle polarizer for broadband infrared application," Appl. Optics, vol. 37, no. 7, p. 1194, 1998 - 1204.

[82] J.-S. Li, D.-g. Xu and J.-q. Yaoq, "Compact terahertz wave polarizing beam splitter," Appl. Optics, vol. 49, no. 24, pp. 4494 - 4497, 2010.

[83] J. C. Martínez - Antón and E. Bernabeu, "High performance Feussner-type polarizers based on stretched poly(ethyleneterephthalate) films," Appl. Phys. Lett., vol. 80, no. 10, pp. 1692 - 1694, 2002.

[84] C. -. F. Hsieh, Y. -. C. Lai, R. -. P. Pan and C. -. L. Pan, "Polarizing terahertz waves with nematic liquid crystals," Opt. Lett., vol. 33, no. 11, pp. 1174 - 1176, 2008.

[85] A. Wojdyla and G. Gallot, "Brewster's angle silicon wafer terahertz linear polarizer," Opt. Express, vol. 19, no. 15, pp. 14099 - 14107, 2011.

[86] E. Hecht, OPTICS - Fourth Edition, Internation Edition: Adelphi University, 2002.

[87] Y. Ekinci, H. H. Solak, C. David and H. Sigg, "Bilayer Al wire-grids as broadband and high - performance polarizers," Opt. Express, vol. 14, no. 6, pp. 2323 - 2334, 2006.

[88] K. Imakita, T. Kamada, M. Fujii, K. Aoki, M. Mizuhata and S. Hayashi, "Terahertz wire grid polarizer fabricated by imprinting porous silicon," Opt. Lett., vol. 38, no. 23, pp. 5067 - 5070, 2013.

[89] K. Shiraishi, S. Higuchi, K. Muraki and H. Yoda, "Silver-film subwavelength gratings for polarizers in the terahertz and mid-infrared regions," Opt. Express, vol. 24, no. 18, pp. 20177 - 20186, 2016.

[90] I. Yamada, K. Takano, M. Hangyo, M. Saito and W. Watanabe, "Terahertz wire-grid polarizers with micrometer-pitch Al gratings," Opt. Lett, vol. 34, no. 3, p. 103206, 2009. 
[91] Z. Huang, E. P. J. Parrott, H. Park, H. P. Chan and E. Pickwell-MacPherson, "High extinction ratio and low transmission loss thin-film terahertz polarizer with a tunable bilayer metal wire-grid structure," Opt. Lett., vol. 39, no. 4, p. 793, 2014.

[92] J. R. Middendorf, J. S. Cetnar, J. Owsley y E. R. Brown, «High fill factor substrate-based wire-grif polarizers with high extinction ratios, » Trans. IEEE THz Sci and Tech., vol. v4, no 3, pp. 376 - 386, 2014.

[93] I. Yamada, N. Yamashita, K. Tani, T. Einishi, M. Saito, K. Fukumi and J. Nishii, "Infrared Wire-Grid Polarizer with Antireflection Structure by Imprinting on Both Sides," Appl. Phys. Express, vol. 5, no. 082502, pp. 1 - 3, 2012.

[94] S. -. W. Ahn, K. -. D. Lee, J. -. S. Kim, S. -. H. Kim, J. -. D. Park, S. -. H. Lee and P. -. W. Yoon, "Fabrication of a $50 \mathrm{~nm}$ half-pitch wire grid polarizer using nanoimprint lithography," Nanotechnology, vol. 16, p. 1874-1877, 2005.

[95] J. S. Cetnar, J. Middendorf and E. R. Brown, "Extraordinary Optical Transmission and Extinction in a Terahertz Wire-Grid Polarizer," Appl. Phys. Lett., vol. 100, no. 23, p. 231912, 2012.

[96] D. -. B. Tian, H. -. W. Zhang, W. -. E. Lai, Q. -. Y. Wen, Y. -. Q. Song and Z. -. G. Wagn, "Double Wire-Grid Terahertz Polarizer on Low-Loss Polymer Substrates," Chin. Phys. Lett., vol. 27, no. 10, pp. 104210 - 1 - 104210 - 3, 2010.

[97] D. B. Fast, T. D. Gerke, Y. Goncharov, S. P. Lebedev, G. Komandin and V. G. Kozlov, "Wire-Grid THz Polarizers manufactured by laser micromachining of metal films on a polymer membrane," International Conference on Infrared, Millimeter, and Terahertz Waves,, pp. 1 - 2, 2011.

[98] K. Shiraishi and K. Muraki, "Metal-film subwavelength-grating polarizer with low insertion losses and high extinction ratios in the terahertz region," Opt. Express, vol. 23, no. 13, pp. 16676 - 16681, 2015.

[99] A. Farid, N. . J. Laurita, B. Tehrani, J. G. Hester, M. M. Tentzeris and N. P. Armitage, "Inkjet Printed Wire-Grid Polarizers for the THz Frequency Range," J. Infrared Milli. Terahz. Waves, vol. 38, p. 276-282, 2017.

[100] A. Partanen, J. Väyrynen, S. Hassinen, H. Tuovinen, J. Mutanen, T. Itkonen, P. Silfsten, P. Pääkkönen, M. Kuittinen, K. Mönkkönen and T. Venäläinen, "Fabrication of terahertz wire-grid polarizers," Appl. Opt., vol. 51, no. 35, pp. 8360-8365, 2012.

[101] A. Ferraro, D. C. Zografopoulos, M. Missori, M. Peccianti, R. Caputo and R. Beccherelli, "Flexible terahertz wire grid polarizer with high extinction ratio and low loss," Opt. Letters, vol. 41, no. 9, pp. 2009 - 2012, 2016.

[102] W. Lai, H. Yuan, H. Fang, Y. Zhu and H. Wu, "Ultrathin, highly flexible and optically transparent terahertz polarizer based on transparent conducting oxide," J. Phys. D: Appl. Phys., vol. 53, no. 125109, pp. 1 - 6, 2020.

[103] M. Colleoni and B. Vidal, "Analysis of the THz response of a simple periodic graphitebased structure," Opt. Express, vol. 22, no. 24, pp. 30156 - 30160, 2014.

[104] Y. Gao, H. Li and J. Liu, "Direct writing of flexible electronics through room temperature liquid metal ink," PLOS ONE, vol. 7, no. 9, p. e45485, 2012.

[105] "Resistivity and temperature coefficient at $20{ }^{\circ} \mathrm{C}, "$ [Online]. Available: http://hyperphysics.phy-astr.gsu.edu/hbase/tables/rstiv.html. [Accessed 2014].

[106] L. Y. Deng, J. H. Teng, L. Zhang, Q. Y. Wu, H. Liu, X. H. Zhang and S. J. Chua, "Extremely high extinction ratio terahertz broadband polarizer using bilayer subwavelength metal wire-grid structure," Appl. Phys. Lett., vol. 101, no. 011101, pp. 1 - 4, 2012.

[107] B. Lu, H. Wang, J. Shen, J. Yang, H. Mao, L. Xia, W. Zhang, G. Wang, X.-Y. Peng and D. Wang, "A high extinction ratio $\mathrm{THz}$ polarizer fabricated by double-bilayer wire grid structure," AIP Advances, vol. 6, no. 025215, pp. 1 - 6, 2016.

[108] A. Isozaki, T. Kan, K. Takano, M. Hangyo, K. Matsumoto and I. Shimoyama, "Doublelayer wire grid polarizer for improving extinction ratio," Transducers 2013 Barcelona, pp. $530-533,2013$. 
[109] D. Tian, X. Dong, Q. Wen, W. Lei and H. Zhang, "Terahertz transmission characteristics of double wire-grid polarizer on low-loss polyethylene substrate," Appl Phys B, vol. 104, p. 125-130, 2010.

[110] Y. Kishi, M. Nagai, J. C. Young, K. Takano, M. Hangyo and T. Suzuki, "Terahertz laminated-structure polarizer with high extinction ratio and transmission power," Appl. Phys. Express, vol. 8, no. 032201, pp. 1 - 4, 2015.

[111] T. -. Y. Yu, N. -. C. Chi, H. -. C. Tsai, S. -. Y. Wang, C. -. W. Luo and K. -. N. Chen, "Robust terahertz polarizers with high transmittance at selected frequencies through $\mathrm{Si}$ wafer bonding technologies," Opt. Lett., vol. 42, no. 23, pp. 4917 - , 2017.

[112] K. Takano, T. Kawabata, C.-F. Hsieh, K. Akiyama, F. Miyamaru, Y. Abe, Y. Tokuda, R.-p. Pan, C.-L. Pan and M. Hangyo, "Fabrication of Terahertz Planar Metamaterials Using a Super-Fine Ink-Jet Printer," Appl. Phys. Express, vol. 3, pp. 016701-1 - 016701-3, 2010.

[113] S. H. Ahn, J. -. S. Kim and L. J. Guo, "Bilayer metal wire-grid polarizer fabricated by rollto-roll nanoimprint lithography on flexible plastic substrate," J. Vac. Sci. Technol. B, vol. 25, no. 6, pp. 2388 - 2391, 2007.

[114] L. Chen, J. J. Wang, F. Walters, X. Deng, M. Buonanno, S. Thai and X. Liu, "Large flexible nanowire grid visible polarizer made by nanoimprint lithography," Appl. Phys. Lett., vol. 90, p. 063111, 2007.

[115] K. Takano, H. Yokoyama, A. Ichii, I. Morimoto and M. Hangyo, "Wire-grid polarizer sheet in the terahertz region fabricated by nanoimprint technology," Opt. Letters, vol. 36, no. 14, pp. 2665 - 2667, 2011.

[116] X.-R. Huang and R.-W. Peng, "General mechanism involved in subwavelength optics of conducting microstructures: charge-oscillation-induced light emission and interference," J. Opt. Soc. Am. A, vol. 27, no. 4, pp. 718 - 729, 2010.

[117] F. J. García-Vidal, L. Martin-Moreno, T. W. Ebbesen and L. Kuipers, "Light passing through subwavelength apertures," Rev. Mod. Plasma Phys., vol. 82, no. 1, pp. 729 - 787, 2010.

[118] J. J. Wang, F. Walters, X. Liu, P. Sciortino and X. Deng, "High-performance, large area, deep ultraviolet to infrared polarizers based on $40 \mathrm{~nm}$ line/78 nm space nanowire grids," Appl. Phys. Lett., vol. 90, no. 061104, pp. 1 - 3, 2007.

[119] A. M. Helmenstine, "Table of Electrical Resistivity and Conductivity," ThoughtCo, 112 2020. [Online]. Available: thoughtco.com/table-of-electrical-resistivity-conductivity608499.. [Accessed 0105 2020].

[120] G. Ramakrishnan, R. Chakkittakandy and P. C. M. Planken, "Terahertz generation from graphite," Opt. Express, vol. 17, no. 18, pp. 16092 - 16099, 2009.

[121] T. Hattori, H. Kumon and H. Tamazumi,, "Terahertz Spectroscopic Characterization of Paper," 35th International Conference on Infrared, Millimeter, and Terahertz Waves, Rome, Italy, 5-10 September, pp. 1 - 2, 2010.

[122] E. Abraham, A. Younus, A. El Fatimy,, J. C. Delagnes, E. Nguéma and P. Mounax, "Broadband terahertz imaging of documents written with lead pencils," Opt. Commun., vol. 282, no. 15, p. 3104-3107, 2009.

[123] F. Yan, C. Yu, H. Park, E. Parrott and E. Pickwell-MacPherson, "Advances in polarizer technology for terahertz frequency applications," J. Infrared Milli Terahz Waves, vol. 34, no. 9, p. 489-499, 2013.

[124] N. R. Center, "The Physics FactBoook," [Online]. Available: https://www.nde-ed.org/. [Accessed 2014].

[125] A. Das, T. Schutzius, C. M. Megaridis, S. Subhechha, T. Wang and L. Liu, "Quasi-optical polarizers enabled by Inkjet Printing of Carbon Nanocomposites," Appl. Phys. Lett., vol. 101, no. 24, pp. 1 - 5, 2012.

[126] Y. Zheng, Q. Zhang and J. Liu, "Pervasive liquid metal direct writing electronics with roller-ball pen," AIP Adv., vol. 3, p. 112117, 2013. 
[127] Q. Xu, N. Oudalov, Q. Guo, H. M. Jaeger and E. Brown, "Effect of oxidation on the mechanical properties of liquid gallium and eutectic gallium-indium," AIP Phys. Fluids (1994-present), vol. 24, p. 063101, 2013.

[128] "Rotring," [Online]. Available: http://www.rotring.com/en/93-technicalcalligraphycalligraphy-pens/93-calligraphy-pens-technical. [Accessed 2015]

[129] M. D. Dickey, "Emerging application of liquid metals featuring surface oxides," Appl. Mater. Interfaces, vol. 6, no. 21, pp. 18369 - 18379, 2014.

[130] M. D. Dickey, R. J. Larsen, E. A. Weiss, D. A. Weitz and G. M. Whitesides, "Eutectic Gallium-Indium (EGaln): a liquid metal alloy for formation of stable structures in microchannels at room temperature," Adv. Funct. Mater., vol. 18, p. 1097-1104, 2008.

[131] F. M. Smits, "Measurements of sheet resistivities with the four-point method probe," Bell System Technical Journal,, vol. 34, p. 711-718, 1957.

[132] D. Kim and E. Sim, "Segmented coupled-wave analysis of a curved wire - grid polarizer," J. Opt. Soc. Am. A, vol. 25, no. 3, p. 558-565, 2008.

[133] Y. Zheng, Z. Z. He, J. Yang and J. Liu, "Personal electronics printing via tapping mode composite liquid metal ink delivery and adhesion," Sci. Rep., vol. 4, no. 4588, pp. 1 - 8, 2014.

[134] S. Brinkmann, N. Vieweg, G. Gärtner, P. Plew and A. Deninger, "Towards Quality Control in Pharmaceutical Packaging: Screening Folded Boxes for Package Inserts," J. Infrared Milli. Terahz Waves, vol. 38, pp. 339 - 346, 2017.

[135] J. Palací and B. Vidal, "Distributed THz Transmitter/Receiver based on a $1.5 \mu \mathrm{m}$ Fiber Link," 36th International Conference on Infrared, Millimeter and Terahertz Waves (IRMMW-THz), Houston (USA), 2-7 October 2011.

[136] E. g. Crizaf.srl, "http://www.crizaf.com," [Online].

[137] J. A. Arnaud, W. M. Hubbard, G. D. Mandeville, B. de la Clavière, E. A. Franke and J. M. Franke, "Technique for fast measurement of gaussian laser beam parameters," Appl. Optics, vol. 10, no. 12, pp. 1 - 2, 1971.

[138] A. H. Firester, M. E. Heller and P. Sheng, "Knife-edge scanning measurements of subwavelength focused light beams," Appl. Optics, vol. 16, no. 7, pp. 1971 - 1974, 1977.

[139] J. M. Khosrofian and B. A. Garetz, "Measurement of a Gaussian laser beam diameter through the direct inversion of knife-edge data," Appl. Optics, vol. 22, no. 21, pp. 3406 3410, 1983.

[140] Z. Jiang and X.-C. Zhang, "2D measurement and spatio-temporal coupling of few-cycle THz pulses," Opt. Express, vol. 5, no. 11, pp. 243 - 248, 1999.

[141] S. Mickan, D. Abbott, J. Munch, X. -C. Zhang and T. van Doorn, "Analysis of system trade-offs for terahertz imaging," MICROELECTRON J, vol. 31, no. 7, pp. 503 - 514, 2000.

[142] TERASENSE, "TERASENSE," [Online]. Available: https://terasense.com/products/subthz-imaging-cameras/. [Accessed 0205 2020].

[143] i2S, “i2S," i2S, [Online]. Available: https://www.i2s.fr/en/product/tzcam.

[144] N. E. b. innovation, "NEC Empowered by innovation," [Online]. Available: http://www.ucinstruments.com/PDF/NEC/THzIRV0831e-1.pdf. [Accessed 0205 2020].

[145] J. Magnes, D. Odera, J. Hartke, M. Fountain, L. Florence and V. Davis, "Quantitative and Qualitative Study of Gaussian Beam Visualization Techniques," arXiv:physics/0605102 [physics.optics], 2008.

[146] O. Mata - Mendez, M. Cadilhac and R. Petit, "Diffraction of a two-dimensional electromagnetic beam wave by a thick slit pierced in a perfectly conducting screen," J. Opt. Soc. Am, vol. 73, no. 3, pp. 328 - 331, 1983.

[147] O. Mata-Mendez, "Diffraction and beam-diameter measurement of Gaussian beams at optical and microwave frequencies," Opt. Lett., vol. 16, no. 21, pp. 1629 - 1631, 1991.

[148] R. L. McCally, "Measurement of Gaussian beam parameters," Appl. Optics, vol. 23, no. 14 , p. 2227, 1984. 
[149] M. Mylonakis, S. Pandey, K. G. Mavrakis, G. Drougakis, G. Vasilakis, D. G. Papazoglou and W. von Klitzing, "Simple precision measurements of optical beam sizes," Appl. Opt., vol. 57, no. 33, pp. 9863-9867, 2018.

[150] J. F. Molloy, M. Naftaly and R. A. Dudley, "Characterization of terhaertz beam profile and propagation through complex quasi-optic systems," Terahertz Science and Technology, vol. 4, no. 3, pp. 99 - 103, 2011.

[151] M. Peccianti, M. Clerici, A. Pasquazi, L. Caspani, S. Phing Ho, F. Buccheri, J. ALi, A. Busacca, T. Ozaki and R. Morandotti, "Exact reconstruction of THz sub- $\lambda$ source features in Knife-Edge measurements," IEEE J. Sel. Top. Quantum Electron, vol. 19, no. 1, p. 8401211, 2013.

[152] A. Bitzer, M. Walther, A. Kern, S. Gorenflo and H. Helm, "Examination of the spatial and temporal field distributions of single-cycle terahertz pulses at a beam focus," Appl. Phys. Lett., vol. 90, no. 071112, pp. 1 - 3, 2007.

[153] P. U. Jepsen and S. R. Keiding, "Radiation patterns from lens-coupled terahertz antennas," Opt. Lett., vol. 20, no. 8, pp. 807 - 809, 1995.

[154] A. Bitzer, H. Helm and M. Walther, "Beam-Profiling and Wavefront-Sensing of THz Pulses at the Focus of a Substrate-Lens," IEEE J. Sel Top. Quant., vol. 14, no. 2, pp. 476 481, 2008.

[155] E. Kholoud, K. Demir, H. Altan, A. B. Sahin and M. Unlu, "Time-Domain Characterization of the Radiation Pattern of the Terahertz Photoconductive Antennas," J. Infrared Millim. Terahertz Waves, vol. 40, pp. 595 - 605, 2019.

[156] J. F. Molloy, M. Naftaly and R. A. Dudley, "Characterization of Terahertz Beam Profile and Propagation," IEEE J. Sel. Top. Quantum Electron, vol. 19, no. 1, 2013.

[157] P. Marchenko, S. Orlov, C. Huber, P. Banzer, S. Quabis, U. Peschel and G. Leuchs, "Interaction of highly focused vector beams woth a metal knife-edge," Opt. Express, vol. 19, no. 8, pp. 7244 - 7261, 2011.

[158] Y. Wang, Z. Zhao, L. Chen, L. Zhang and K. Kang, "Analysis of terahertz Pulse propagation thorugh a dielectric edge," Proc. of SPIE, vol. 7277, no. 72770R, pp. $1-8,2009$.

[159] J. D. Barnett and J. F. S. Harris, "Test of the effect of edge parametrs on small angle fresnel diffraction of light at a straight edge," J. Opt. Soc. Am, vol. 52, no. 6, pp. 637 - 643, 1962.

[160] J. Bromage, S. Radic, G. Agrawal and C. Stroud Jr., "Spatiotemporal shaping of terahertz pulses," Opt. Lett., vol. 22, no. 9, pp. 627 - 629, 1997

[161] A. I. Khizhnyak, S. P. Anokhov, R. A. Lymarenko, M. S. Soskin and M. V. Vasnetsov, "Structure of an edge-dislocation wave originating in plane-wave diffraction by a halfplane," J. Opt. Soc. Am. A, vol. 17, no. 12, pp. 2199 - 2207, 2000.

[162] S. P. Anokhov, "Plane wave diffraction by a perfectly transparent half-plane," J. Opt. Soc. Am. A., vol. 24, no. 9, pp. 2493 - 2498, 2007.

[163] F. Alves, D. Grbovic, B. Kearney and G. Karunasiri, "Microelectromechanical systems bimaterial terahertz sensor with integrated metamaterial absorber," Opt. Lett., vol. 37, no. 11, pp. 1886 - 1888, 2012.

[164] B. Kearney, F. Alves, D. Grbovic and G. Karunasiri, "Al/SiOx/Al single and multiband metamaterial absorbers for terahertz sensor applications," Opt. Eng., vol. 52, no. 1, p. 013801, 2013.

[165] F. Alves, D. Grbovic, B. Kearney, N. V. Lavrik and G. Karunasiri, "Bi - material terahertz sensors using metamaterial structures," Opt. Express, vol. 21, no. 11, pp. 13256 - 13271, 2013.

[166] O. Ajakaiye, J. Grade, S. Choongsoon and T. Kenny, "Wafer-scale fabrication of infrared detectors based on tunneling displacement transducers," Sensors Actuators A, vol. 134, no. 2, pp. 575 - 581, 2007.

[167] D. Molter, L. Ellrich, T. Weinland, S. George, M. Goiran, F. Keilmann, R. Beigang and J. Léotin, "High speed terahertz time-domain spectroscopy of cyclotron resonance in pulsed magnetic field," Opt. Express, vol. 18, no. 25, p. 26163 - 26168, 2010. 
[168] N. Vieweg, F. Rettich, A. Deninger, H. Roehle, R. Dietz, T. Göbel and M. Schell, "Terahertz-time domain spectrometer with $90 \mathrm{~dB}$ peak dynamic range," J. Infrared Millim. TerahertzWaves, vol. 35, pp. 823 - 832, 2014.

[169] T. W. Kenny, J. K. Reynolds, J. A. Podosek, E. C. Vote, L. M. Miller, H. K. Rockstad and W. J. Kaiser, "Micromachined infrared sensors using tunneling displacement transducers," Rev. Sci. Instrum., vol. 67, no. 1, p. 112, 1996.

[170] L. Hou and W. Shi, "An LT-GaAs Terahertz Photoconductive Antenna With High Emission Power, Low Noise, and Good Stability," IEEE T. Electron Dev., vol. 60, no. 5, pp. 1619 - 1624, 2013.

[171] S. Lepeshov, A. Gorodetsky, A. Krasnok, N. Toropov, T. A. Vartanyan, P. Belov, A. Alú and E. U. Rafailov, "Boosting Terahertz Photoconductive Antenna Performance with Optimised Plasmonic Nanostructures," Sci. Rep., vol. 8, no. 6624, pp. 1 - 7, 2018.

[172] P. Huber, P. Martinez, C. Guers, F. Garet and P. Borel, "Dielectric Losses of Paper in the THz Domain: Literature Review, Needs for Future Research, and Prospective Solutions," Phys. Status Solidi, vol. 214, no. 1700356, pp. 1 - 5, 2017.

[173] Z. Y. Yang, M. Zhao, N. L. Dai, G. Yang, H. Long, Y. H. Li and P. X. Lu, "Broadband Polarizers Using Dual-Layer Metallic Nanowire Grids," IEEE Photonic Tech. L., vol. 20, no. 9, pp. 697 - 699, 2008.

[174] P. Klarskov, A. C. Strikwerda, K. Iwaszczuk and P. U. Jepsen, "Experimental threedimensional beam profiling and modeling of a terahertz beam generated from a two-color air plasma," New J. Phys., vol. 15, no. 075012, pp. 1 - 3, 2013.

[175] M. A. C. de Araújo, R. Silva, E. de Lima, D. P. Pereira and P. C. de Oliveira, "Measurement of Gaussian laser beam radius using the knife-edge technique: improvement on data analysis," Appl. Opt., vol. 48, no. 2, pp. 393 - 396, 2009. 
Acronyms and abbreviations

$a$-Si. Amorphous Silicon

Au. Gold

BCB. Benzocyclobutene

BWO. Backward Wave Oscillator

CCD. Charge Coupled Devices

CNT. Carbon Nanotube

COC. Cyclo Olefin Copolymer

Cr. Cromium

CW. Continuous Wave

DCF. Dispersion Compensating Fibers

DFG. Difference Frequency Generation

DOP. Degree of Polarization

D-wave. Dislocation Edge

EDW. Edge Dislocation Wave

EGaln. Eutectic Gallium Indium alloy

EMA. Effective Medium Approximation

EO. Electro Optical

ER. Extinction Ratio

FDTD. Finite Difference Time Domain

FEL. Free Electron Laser

IL. Insertion Loss 
ITO. Tin doped indium oxide

KE. Knife Edge

LO. Longitudinal Optical

MWCNT. Multi-Walled Carbon Nanotube

Ni. Nichel

NLC. Nematic Liquid Crystal

OCT. Optical Coherence Tomography

ODL. Optical Delay Line

OPD. Optical Path Difference

OPL. Optical Path Length

PC. Photoconductive

PET. Polyethylene terephthalate

PP. Polypropylene

PVA. Polyvinyl Alcohol

QCL. Quantum Cascade Lasers

SMF. Single Mode Fibers

SOP. State of Polarization

SPP. Surface Plasmons Polaritons

SWGP. Single Wire Grid Polarizer

TDS. Time Domain Spectroscopy

TE. Transverse Electric

TM. Transverse Magnetic

TPX. Polymethylpentene

WGP. Wire Grid Polarizer 
Publications derived from the Thesis

\section{Publications in indexed journals}

- M. P.M Colleoni and B. Vidal, "Analysis of the THz response of a simple periodic graphite-based structure," Opt. Express, vol. 22, no. 24, pp. 30156 - 30160, 2014.

- M.P. M. Colleoni and B. Vidal, "Flexible Sub-THz Metal Wire Grid Polarizer Based on an EGaln 24.5 Alloy," IEEE Transactions on Terahertz Science and Technology, vol. 6, no. 5, pp. 757-759, Sept. 2016.

- M.P.M. Colleoni, M.A. Báez-Chorro, B. Vidal, “Quantitative Characterization of Defects with Fixed-Delay THz Sensors", IEEE Sensors Letters, vol. 3, no. 10, pp. 3501804, October 2019.

\section{Publications in international conferences:}

- M. P.M Colleoni, Q. Wang, J. Liu, and B. Vidal "Printing sub-THz wire grid polarizers using a composite liquid metal ink", Proc. SPIE 10103, Terahertz, RF, Millimetre, and Submillimetre-Wave Technology and Applications X, 101031T , February 2017. 
I. Annex-Mathematical study of diffraction on the

slit

Despite the Huygens approach, in which the propagation of a front wave is seen as the superposition of the emission of secondary sources, a different approach was followed to describe the diffraction of a wave by a metal edge. In this theory, diffraction could be described as the superposition of a scattered edge wave or boundary wave, i.e. the obstructed part of the light and a geometrical wave, that is the unobstructed original wave. Sommerfeld first addressed this problem and rigorously solved mathematically the diffraction of a monochromatic wave by a perfectly conducting infinite half - plane. This solution had been largely recognized and several researchers refined it during the past century. However, Khizhnyak et al. 2000 [161] pointed out that there were still loose ends, since the Sommerfeld components of the wave, both the boundary and the geometrical, could not be real and propagate as real waves. Hence, Khizhnyak et al. 2000 filled the gap by describing the diffraction field behind the metal edge at a point $\mathrm{P}, E_{Z}(P)$, as a superposition of two wave components, one being the half amplitude of the incident plane wave and the other named edge dislocation wave (EDW) that contained all the information about the diffraction field (equation 6 of [161]):

$$
E_{Z}(P)=A_{0} e^{-i k z}\left[\frac{1}{2}+\frac{1-i}{\sqrt{2 \pi}}\right] \int_{0}^{U} e^{i \mu^{2}} d \mu
$$


where $U$ is the scalar length $U=\sigma\left\{k \sqrt{\left(\sqrt{\left(x^{2}+z^{2}\right)+z}\right)}\right\}$ and $\sigma$ is negative in the plane that contains the edge (the blue quadrant) and positive otherwise. This result is reproduced in Fig. 47 a).

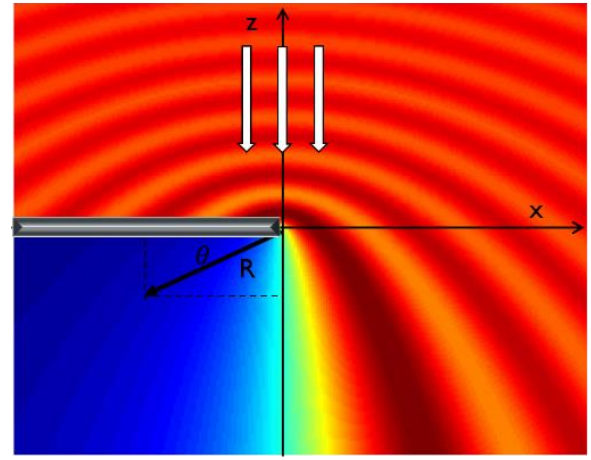

(a)

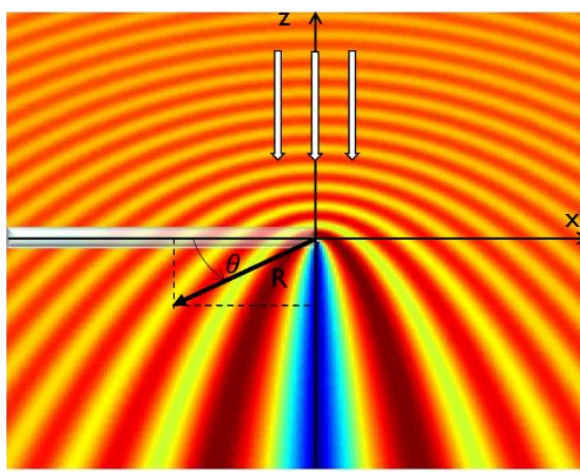

(b)

Fig. 47: Simulation of diffraction of a monochromatic wave by a semi-plane perfectly conducting (a) and dielectric (b) with $\varphi=\pi$ phase step. The wave travels from top to bottom and impinges on the edge

The same approach was later applied by Anokhov et al. in 2007 [162], where the perfectly conducting half plane was replaced by an infinite, optical thin dielectric edge. As in the first case, the resulting wave behind the edge was again the combination of two components, like those found in the case of the metal razor, albeit in this second case, neither absorbance nor reflection were considered, being a phase step the only effect induced by the edge on the wave. The signal power was preserved as it was completely transmitted, but the pulse underwent recombination, due to the interaction between the augend wave and the addend wave. The former was still a copy of the incoming wave with halved amplitude, whereas the second was the aforementioned EDW or dislocation wave (D-wave), since it had a dislocation edge on its front. The field in the plane behind the edge at a point $\mathrm{P}$ is reproduced in Fig. $47 \mathrm{~b}$ ) for the case of normal incidence $\alpha=\frac{\pi}{2}$ and it is described by the following equation (equation 14 in [162]):

$$
E_{\Sigma}(P)=A_{0} e^{-1 k R \sin \theta}\left\{\frac{1+e^{i \varphi}}{2}+\left[1-e^{i \varphi}\right] \frac{1-i}{\sqrt{2 \pi}} \int_{0}^{U} e^{i \mu^{2}} d \mu\right\}
$$


Where in this case $U=\sqrt{2 k R} \sin \left[\frac{(\theta-\alpha)}{2}\right]$ in cylindrical coordinates and $\operatorname{sign} U$ is determined by the value of the function $\sin \left[\frac{(\theta-\alpha)}{2}\right]$. Therefore, $U>0$ for $\theta-$ $\alpha>0$ and negative otherwise.

There was a strong dependence between the wavelength of the incoming wave and the half plane thickness (or height), that led to three possible situations:

- $\varphi=0 ; \varphi=2 \pi$ : no delay is induced, and the wave passes unaltered.

- $\varphi=\pi$ : the augend disappears and only the augend propagates behind the dielectric step. This specific wave has a dislocation edge in its front and it is called $d$-wave (diffraction and dislocation wave)

- $0<\varphi<\pi ; \pi<\varphi<2 \pi$ : the resulting field is the combination of the types of wave, with an energy ratio that varies according to the delay $\varphi$.

These theoretical results were applied by [158] to qualitative explain the enhanced diffraction edge effect that allowed to clearly distinguish a T shape cut in a dielectric sample, when illuminating with a pulsed THz beam, in spite of being made of the same material of the background sample. Again, the dielectric step induced a recombination of the $\mathrm{THz}$ pulse that crossed it, due to a phase delay that depended on the step thickness. Moreover, the presence of a dielectric material in the beam path induced a specific delay in each frequency component, and the pulse experimented a small deformation due to its recombination behind the slit area.

Formula (3) of Wang et al., 2009 [158] allowed to control the phase delay of each frequency component:

$$
\varphi=\frac{(n-1) \omega d}{c}
$$

The proper dielectric thickness $d$ or height plays the twofold role of ensuring to deal with a Gaussian beam in the focus plane, by being smaller than the Rayleigh length, and to know the selected frequency components that are going to suffer a phase delay of $\pi$.

Results are gathered for the specific case of the slit simulation instead of the edge ones. Simulations with different frequencies for both materials with a fixed $0.5 \mathrm{~mm}$ slit width are proposed in Table 1. In particular, the first line represents the case of a phase delay $\varphi$ that lies between $0<\varphi<0.9 \pi$, the second line is the case $\varphi=0.9 \pi$ (corresponding to the $d$-wave condition in this case) and the last line is the frequency that corresponds to the $\varphi=1.5 \pi$. 
WAVE

FREQUENCY

METAL

DIELECTRIC

(THz)
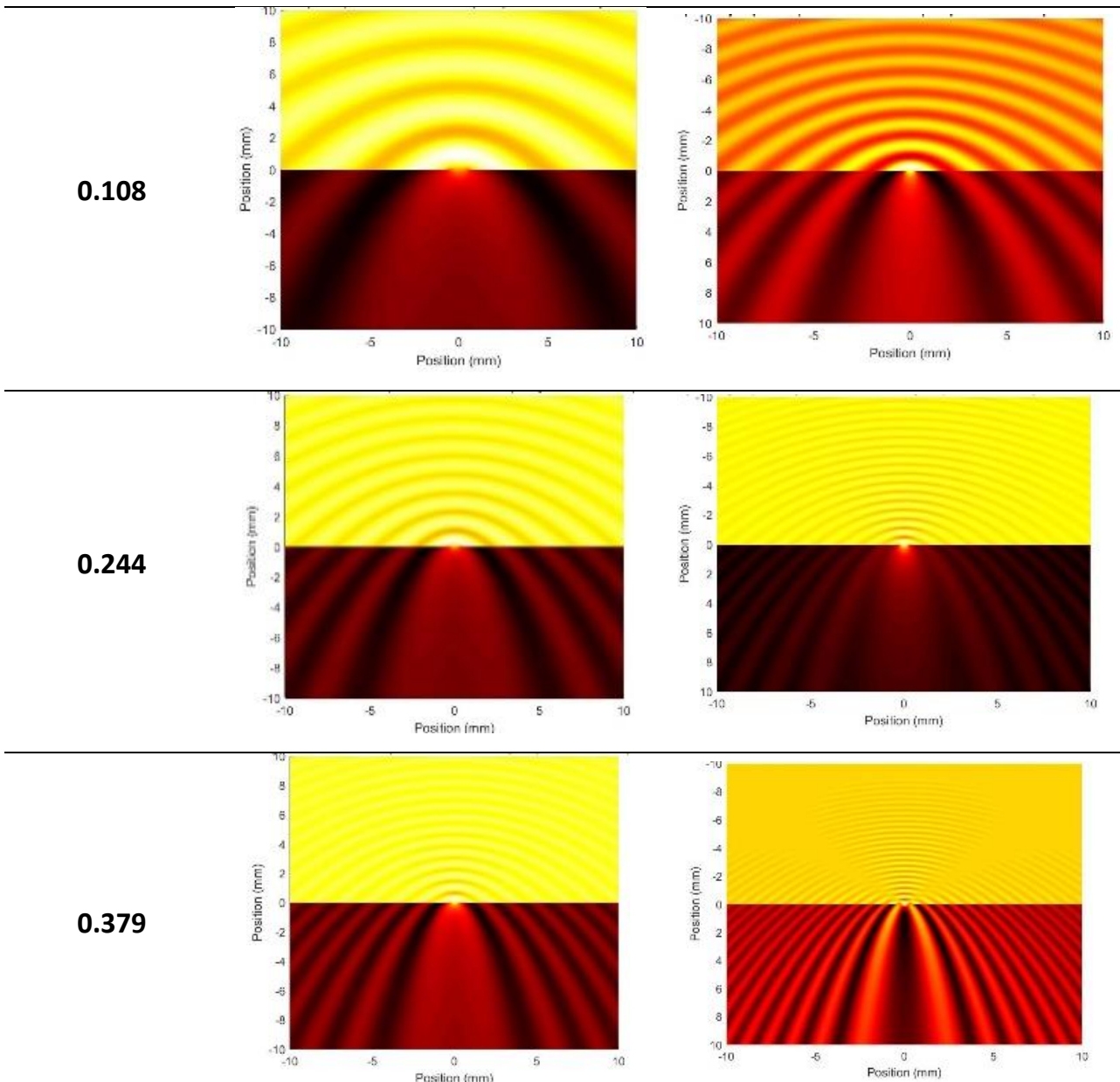

Table 1:Results of the simulation of THz pulses with frequencies $0.108 \mathrm{THz}, 0.244 \mathrm{THz}, 0.379 \mathrm{THz}$ for a slit width of $0.5 \mathrm{~mm}$

Simulations clearly show another effect related with this theory that is the modulation of the field of the impinging wave in the region in front of the slit, due to the diffraction effect. In fact, part of the field of the diffracted wave propagates towards the incoming wave. This so-called backward wave has a rapidly decaying amplitude, whose maximum equals $A_{0}$ and it is located at the dielectric edge. 